\title{
WestVirginiaUniversity
}

THE RESEARCH REPOSITORY @ WVU

Graduate Theses, Dissertations, and Problem Reports

2003

\section{Molecular cytogenetic evaluation of uveal melanoma cell lines and archival tissue}

Jason Scott White

West Virginia University

Follow this and additional works at: https://researchrepository.wvu.edu/etd

\section{Recommended Citation}

White, Jason Scott, "Molecular cytogenetic evaluation of uveal melanoma cell lines and archival tissue" (2003). Graduate Theses, Dissertations, and Problem Reports. 1951.

https://researchrepository.wvu.edu/etd/1951

This Dissertation is protected by copyright and/or related rights. It has been brought to you by the The Research Repository @ WVU with permission from the rights-holder(s). You are free to use this Dissertation in any way that is permitted by the copyright and related rights legislation that applies to your use. For other uses you must obtain permission from the rights-holder(s) directly, unless additional rights are indicated by a Creative Commons license in the record and/ or on the work itself. This Dissertation has been accepted for inclusion in WVU Graduate Theses, Dissertations, and Problem Reports collection by an authorized administrator of The Research Repository @ WVU.

For more information, please contact researchrepository@mail.wvu.edu. 


\title{
Molecular Cytogenetic Evaluation of \\ Uveal Melanoma Cell Lines and Archival Tissue
}

\author{
Jason Scott White \\ Dissertation submitted to the \\ College of Agriculture, Forestry and Consumer Sciences \\ West Virginia University \\ in partial fulfillment of the requirements \\ for the degree of \\ Doctor of Philosophy \\ In \\ Genetics \& Developmental Biology \\ Joginder Nath, Ph.D., Chair, WVU \\ Alison E. Director-Myska, Ph.D., AFIP \\ Sharon L. Wenger, Ph.D., WVU \\ Robert L. Becker, Jr., M.D., Ph.D., Col, USAF, MC, AFIP \\ Walter J. Kaczmarczyk, Ph.D., WVU \\ Daniel Pannaccione, Ph.D., WVU \\ Morgantown, West Virginia \\ 2003
}

Keywords: Cytogenetics, Uveal Melanoma, CGH, SKY, FISH

Copyright 2003 Jason S. White 


\section{ABSTRACT \\ Molecular Cytogenetic Evaluation of Cell Lines and Archival Tissue \\ Jason Scott White}

Uveal melanoma is the most common intraocular tumor in adults and often results in unilateral blindness and/or death. Previous cytogenetic characterizations of this tumor have consistently revealed chromosomal abnormalities involving chromosomes 3,6 , and 8; reports of other abnormalities vary in frequency. We further defined cytogenetic abnormalities of this tumor using molecular cytogenetic techniques on 10 uveal malignant melanoma cell lines and 100 formalin-fixed paraffin-embedded (FFPE) tumors.

The synthesis of comparative genomic hybridization (CGH) and spectral karyotyping (SKY) results revealed that chromosomal rearrangement plays a significant role in the generation of DNA sequence copy number abnormalities throughout the genome, but none of the cell lines demonstrated monosomy 3. Centromeric fluorescence in situ hybridization (FISH) for chromosome 3 revealed approximately one signal per cell, but further evaluation with telomeric probes demonstrated multiple signals per cell, suggesting chromosomal rearrangement without the loss of an entire chromosome 3 . Based on combined CGH, SKY and FISH data, we propose that chromosome 3 is more frequently involved in chromosomal rearrangements rather than whole-chromosome loss in uveal melanoma.

CGH was similarly employed to elucidate characteristic DNA-sequence copy number abnormalities of FFPE archival cases of uveal melanoma, and correlate genomic 
imbalance abnormalities with a prognosis. We set out to study 100 archival uveal melanoma cases, each with comprehensive patient follow-up, by correlation of copy number imbalances with survival. Fifty-one patients survived more than 9 years without evident metastasis, and the remaining 49 patients died with metastatic disease. Viable probe was ge nerated from 82 of the 100 cases, allowing correlation of CGH findings with patient histories for all but 18 of the cases. Significant copy number imbalances were tested for univariate prognostic significance. The most powerful predictor of prognosis was gain of 18q11.2, which was subsequently compared with other significant chromosomal regions, as well as histological and clinical factors in a multivariate analysis. Multivariate analyses revealed that the gain of $18 \mathrm{q} 11.2$ and concomitant loss of $1 \mathrm{p} 33$ was the strongest indicator of a poor prognosis.

Our large-scale molecular cytogenetic analysis of cell lines and archival material contributes significantly to the characterization of uveal melanoma. This research is intended to direct further gene-specific study of malignancy in uveal melanoma. 


$$
\text { To mp parents, }
$$

\title{
Soan and ofeal Dhite,
}

\author{
who have made innumerable sacrifices for my ectucation, \\ so $\bigcirc$ could learn without limitations
}




\section{TABLE OF CONTENTS}

Dedications

Table of Contents

$\mathbf{v}$

List of Abbreviations

vi

List of Figures

$\mathbf{X}$

List of Tables

xiii

Acknowledgements

xiv

Introduction

1

Literature Review

12

Materials and Methods

42

Results

71

Discussion

102

Summary

116

References

117

Appendix A

130

Appendix B

139

Vitae

145 


\section{LIST OF ABBREVIATIONS}

\begin{tabular}{ll} 
AAF & aminoacetylfluorene \\
AFIP & Armed Forces Institute of Pathology \\
bP & base pair(s) \\
BSA & bovine serum albumin \\
CaCl 2 & calcium chloride \\
CCD & charge-coupled device \\
CGH & comparative genomic hybridization \\
CISS & chromosomal in situ suppression \\
CO 2 & carbon dioxide \\
cRNA & complementary ribonucleic acid \\
DAPI & 4',6-diamidino-2-phenylindole \\
dATP & 2'-deoxyadenosine 5'-triphosphate \\
dCTP & 2'-deoxycytidine 5'-triphosphate \\
del & deletion \\
der & derivative chromosome \\
dGTP & 2'-deoxyguanosine 5'-triphosphate \\
dH 2 O & distilled water \\
dic & dicentric chromosome \\
DIG & digoxigenin-11-dUTP \\
DMEM & Dulbecco's Modified Eagle's Medium \\
DNA & deoxyribonucleic acid \\
DNA Pol I & DNA Polymerase I \\
DNP & dinitrophenyl \\
dNTP & 2'-deoxynucleotide 5'-triphosphate \\
DOP-PCR & degenerate oligonucleotide primed-polymerase chain reaction \\
dTTP & 2'-deoxythmidine 5'-triphosphate \\
dUTP & 2'-deoxyuridine 5'-triphosphate \\
EDTA & ethylenediaminetetraacetic acid \\
FA & formamide \\
FAM-M & familial atypical mole and melanoma \\
\hline
\end{tabular}




\begin{tabular}{|c|c|}
\hline FBS & fetal bovine serum \\
\hline FFPE & formalin-fixed paraffin-embedded \\
\hline FISH & fluoresence in situ hybridization \\
\hline FITC & fluorescein isothiocyanate \\
\hline $\mathbf{g}$ & gram \\
\hline $\mathbf{H} \& \mathbf{E}$ & hematoxylin and eosin \\
\hline $\mathbf{H}_{2} \mathbf{O}$ & water \\
\hline $\mathrm{HCl}$ & hydrochloric acid \\
\hline HEPES & N-2-Hydroxyethylpiperazine-N'-2-ethanesulfonic acid \\
\hline HPLC & high performance liquid chromatography \\
\hline HPBL & human peripheral blood lymphocytes \\
\hline $\mathbf{i}$ & isochromosome \\
\hline ins & insertion \\
\hline ISH & in situ hybridization \\
\hline $\mathbf{K C l}$ & potassium chloride \\
\hline len1 & $\begin{array}{l}\text { distance, in pixels, representing a unit change in copy number on } \mathrm{x} \text {-axis of } \\
\text { a CGH ratio profile }\end{array}$ \\
\hline LOH & loss of heterozygosity \\
\hline LTD & largest tumor dimension \\
\hline $\mathbf{M}$ & molar concentration \\
\hline $\mathbf{M b}$ & megabase(s) \\
\hline M-FISH & multiplex fluorescence in situ hybridization \\
\hline mg & milligram(s) \\
\hline $\mathrm{MgCl}_{2}$ & magnesium chloride \\
\hline ml & milliliter(s) \\
\hline MM & master mix \\
\hline $\mathbf{m m}$ & millimeter(s) \\
\hline $\mathbf{m M}$ & millimolar \\
\hline $\mathbf{m r}$ & marker chromosome \\
\hline NA & net area \\
\hline $\mathrm{NaCl}$ & sodium chloride \\
\hline
\end{tabular}




\begin{tabular}{|c|c|}
\hline $\mathrm{Na}_{2} \mathrm{CO}_{3}$ & sodium carbonate \\
\hline $\mathrm{NaHCO}_{3}$ & sodium bicarbonate \\
\hline ND & no data \\
\hline NEAA & non-essential amino acids \\
\hline NFDM & non-fat dried milk \\
\hline ng & nanogram(s) \\
\hline NT & nick translation \\
\hline PBS & Dulbecco's Phosphate-buffered saline \\
\hline PCI & phenol/chloroform/isoamyl alcohol \\
\hline PCR & polymerase chain reaction \\
\hline PHA & phytohemagglutinin \\
\hline pg & $\operatorname{picogram}(s)$ \\
\hline PL & profile length \\
\hline PN & $\begin{array}{l}\text { total pixels designated for cytogenetically normal copies of a given } \\
\text { chromosome in a SKY image }\end{array}$ \\
\hline PT & total pixels designated for a given chromosome in a SKY image \\
\hline QCD & quantitative comparison data \\
\hline QPD & quantitative profile data \\
\hline $\mathbf{R B}$ & relative balance \\
\hline $\mathbf{R C E}$ & relative chromosome equivalent \\
\hline rDNA & ribosomal deoxyribonucleic acid \\
\hline RNA & ribonucleic acid \\
\hline rpm & revolutions per minute \\
\hline RPMI & Roswell Park Memorial Institute \\
\hline rRNA & ribosomal ribonucleic acid \\
\hline SAGE & serial analysis of gene expression \\
\hline SDS & sodium dodecyl sulfate \\
\hline SE & standard error \\
\hline SKY & spectral karyotyping \\
\hline SSC & saline-sodium citrate \\
\hline $\mathbf{t}$ & translocation \\
\hline
\end{tabular}


TBE Tris-Borate EDTA

TE Tris-EDTA

TIL tumor-infiltrating lymphocytes

TRITC tetramethylrhodamine isothiocyanate

U unit(s)

UTP uridine 5'-triphosphate

UV ultraviolet

$\mathbf{v} / \mathbf{v} \quad$ volume per volume

w/o without

WVU West Virginia University

$\mu \mathrm{g} \quad \operatorname{microgram}(\mathrm{s})$

$\mu$ microliter(s)

$\mu \mathbf{m} \quad \operatorname{micron}(\mathrm{s})$, micrometer(s)

〜 approximately

3cen chromosome 3 centromere

${ }^{3} \mathbf{H} \quad$ tritium

3p chromosome 3 telomere, p-arm

3q chromosome 3 telomere, q-arm 


\section{LIST OF FIGURES}

1. Anatomy of a Nucleus 2

2. Basic FISH Protocol 4

3. Anatomy of the Ocular Globe 6

4. Examples of Uveal Melanoma Tumors 9

5. Anatomy of the Chromosomes 13

6. Denaturation/Renaturation of DNA 15

7. Comparison of Directly and Indirectly-Labeled Probes 17

8. DOP-PCR Amplification of Genomic DNA 26

9. Schematic for SKY Image Capture and Analysis 34

10. Probe Production via Nick Translation 48

11. Agarose Gel Electrophoresis Comparing DNA Extracts 50 with Nick Translation Products

12. DNA Probe Production via PCR 53

13. A Flowchart for CGH

14. A Schematic for the Process of Hybridization for CGH 55

15. Step-wise Process of CGH Visualization 59

16. Image Capture for CGH Analysis $\quad 60$

17. DNA Counterstain

18. A Schematic for the Process of CGH Analysis 62

19. Quantitative Comparison Data for Genomic Imbalances 72 in Ten Cell Lines

20. Minimal Common Regions of Gain and Loss Distilled from 
CGH Mean Profile Data of Ten Uveal Melanoma Cell Lines

21. Complex Aberrations Detected by Spectral Kary otyping

22. Chromosomal Rearrangements Seen Across Ten Uveal Melanoma Cell Lines

23. Overall Comparison of Calculated CGH RB and SKY RB Values for the 22 Autosomes in Nine Cell Lines

24. Visual Comparison of CGH and SKY

25. Comparisons of FISH, SKY and CGH Results for Chromosomes 3, 6, and 8, the Three Chromosomes Most Frequently Implicated in Uveal Melanoma

26. Visual Comparison of FISH and SKY

27. Minimal Common Regions of Gain and Loss Distilled from CGH Mean Profile Data of 82 FFPE Uveal Melanoma Cases

28. Quantitative Comparison Data and Minimal Common Regions for the Sex Chromosomes of the Male Archival Cases

29. Quantitative Comparison Data and Minimal Common Regions for the Sex Chromosomes of the Female Archival Cases

30. Minimal Common Regions of Gain and Loss Distilled from CGH Mean Profile Data of 43 FFPE Uveal Melanoma Cases, Representing Those Patients that Survived Beyond 9 Years Post-Diagnosis

31. Minimal Common Regions of Gain and Loss Distilled from CGH Mean Profile Data of 39 FFPE Uveal Melanoma Cases, 
Representing Those Patients that Succumbed to

Hematogenous Metastasis

32. Minimal Common Regions for the Sex Chromosomes

33. Univariate Kaplan-Meier Cause Specific Survival

Correlation Curve

34. Multivariate Kaplan-Meier Cause Specific Survival

Correlation Curve

35. Image Capture for CGH Analysis from an Archival Uveal

Melanoma Case

36. An Example of CGH Profile Data

37. Interphase FISH Control Using Centromere Probe for

Chromosome 3

38. Interphase FISH Control Using Telomere Probes for $3 p$ and $3 q$

39. Telomere Probe Signals (3p and 3q) in Uveal Melanoma Cell Lines 


\section{LIST OF TABLES}

1. Uveal Melanoma Cell Lines Used in This Study 8

2. A Comparison of Gains Seen in Ten Uveal Melanoma Cell Lines with Previously Reported Chromosomal

Abnormalities in Uveal Melanoma

3. A Comparison of Losses Seen in Ten Uveal Melanoma

Cell Lines with Previously Reported Chromosomal

Abnormalities in Uveal Melanoma

4. A Correlation of Copy Number Abnormalities in Ten Uveal

Melanoma Cell Lines and Genes Associated with Uveal

Melanoma or its Predisposing Factors

5. Summary of Aberrations Identified by SKY in Ten Uveal Melanoma Cell Lines

6. Chromosome 3 FISH Signals for Ten Uveal Melanoma

Cell Lines

7. Comparison of CGH, SKY and FISH in Relative Balance (RB) and Relative Chromosome Equivalent (RCE) for Chromosomes 3, 6 and 8 


\section{ACKNOWLEDGEMENTS}

First and foremost, I would like to acknowledge Dr. Joginder Nath, Chair, Genetics and Developmental Biology Program, WVU, for always knowing best, and caring so deeply on a professional as well as personal level. Secondly, I would like to thank the faculty and staff of WVU, who have been so helpful throughout my academic tenure. I credit Dr. Sharon Wenger for opening my eyes to human genetics, and always making time for additional instruction and advice. I also thank Dr. Walt Kaczmarczyk for balancing challenging coursework with a note of levity, and Dr. Dan Panaccione for providing instruction and encouragement.

There are numerous individuals that have been of assistance throughout my time at the AFIP. I extend my deepest thanks to Dr. Alison Director-Myska, AFIP, for sticking with me through thick and thin, and for making the learning process a pleasure. I thank Dr. Ian McLean for making this project possible, and Dr. Timothy O'Leary for sharing his expertise and keeping his door open for me. Thanks to Dr. Robert Becker for offering candid evaluations of my work, and always having one more idea. I extend my gratitude to Karen Bijwaard taking time out of her busy schedule for training in molecular techniques, and Dr. Bill (William) Oliver for keen insight into statistical approaches. Special thanks to Annette Geissel, for putting everything in perspective, and Michelle Webb, for putting up with me and helping me through the rough spots. Finally, I thank Dr. Kimberlee Potter for her career advice and for providing a model of perseverance.

To my fellow students at WVU, whose paths I have crossed, I thank you for making me a better person. I thank Dr. Shawna Jackman, my best friend, without whom 
I would not have excelled at WVU, and certainly would not have been able to accomplish this body of work. Thanks also to Dr. Mike Rossi, for setting the standard and never failing to raise the bar, and Jonnie Lane, for breaking the mold and never obeying the norm.

Finally, to Adrian Walleigh, a true friend from beyond the scientific community, who has provided invaluable advice on life and perspective on so many issues. To anyone I may have omitted, my heartfelt apology. You contribution is not forgotten, but my memory misplaced. 


\section{INTRODUCTION}

Our understanding of cancer continues to evolve as we develop new approaches in the pursuit of the elusive cure for this multifaceted disease. With each passing day, either a new technique or a new application of an existing technique propels cancer research. At times it seems that the more that is discovered about cancer, the more questions that are encountered. However, significant progress has been made, and will continue to be made, in this arena.

Pathology is concentrated on the morphology and characteristics of tissue at the cellular level. The advent of cytogenetic analyses provided insight beneath the level of the cell, allowing researchers access to information about the chromosome complement within individual cells (Fig. 1). Classical cytogenetics provides us with a karyotype, or a catalogue of chromosome homologues, and allows for banding as well as copy number analysis. Cytogenetics contributes immensely to cancer research, as chromosome abnormalities are often associated with the mechanisms underlying carcinogenesis as well as other disease pathways. Application of in situ hybridization (ISH) methods to tumor cells yields a variety of information including numerical data and regarding interand intra-chromosomal rearrangements (Cremer et al., 1986; Lichter et al., 1988a; Devilee et al., 1988; Eastmond and Pinkel, 1989; Trask, 1991; Joos et al., 1994). The combination of epifluorescence microscopy and in situ hybridization techniques resulted in fluorescence in situ hybridization (FISH), which is capable of providing chromosome enumeration, whole chromosome painting, marker identification and gene mapping (Chang and Mark, 1997). In brief, FISH is a fairly rapid technique that involves localization of a labeled deoxyribonucleic acid (DNA) sequence to cellular DNA in 


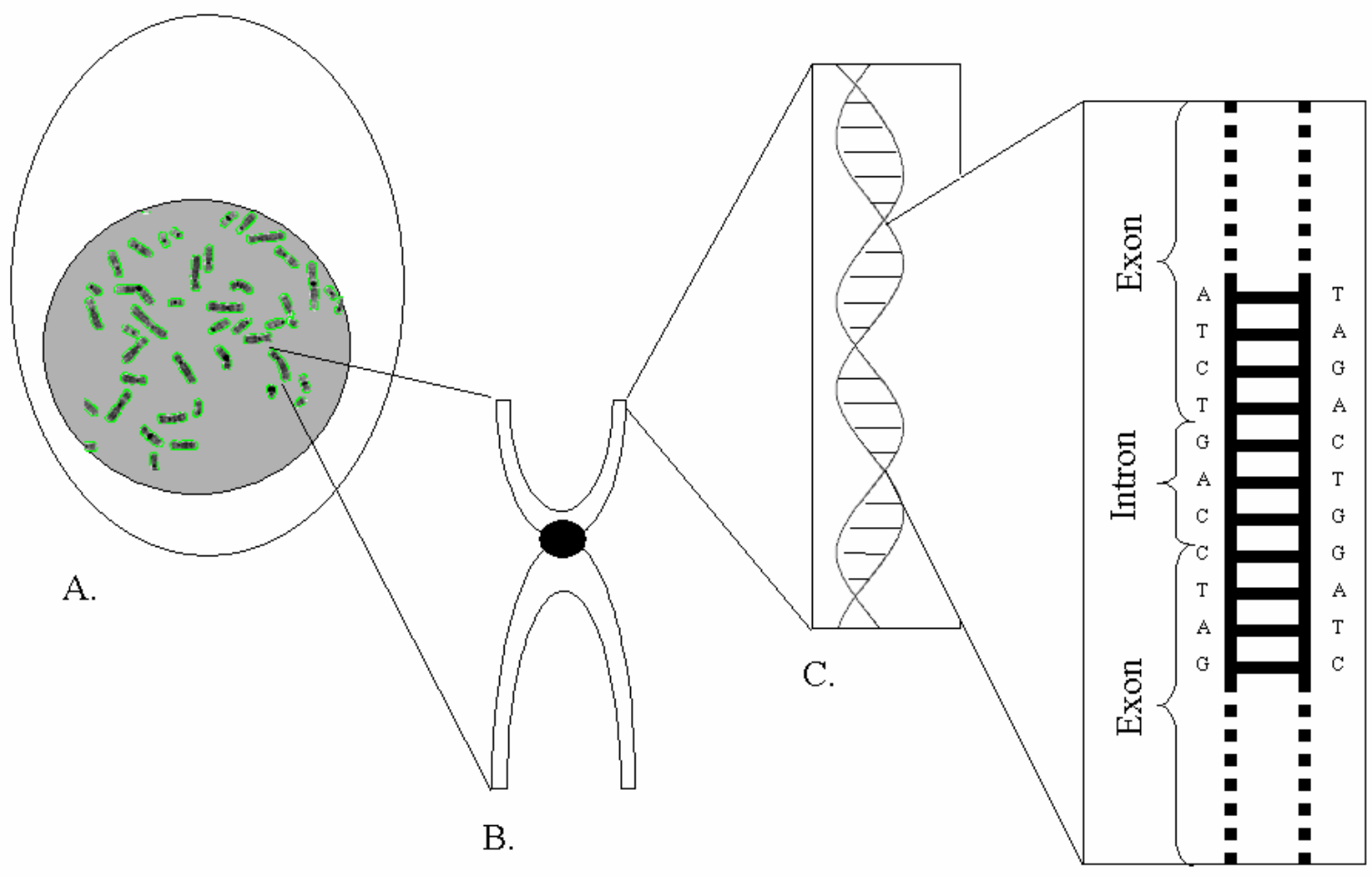

D.

Figure 1. Anatomy of a Nucleus. (A) A stylized drawing of a cell showing a nucleus (gray) containing DNA in the form of chromosomes. (B) A single metaphase chromosome from within the nucleus. (C) Chromosomes are comprised of tightly packed DNA in a double helical conformation. (D) The DNA is made up of nucleotides, of which specific sequences form the exons and introns of genes. 
whole tissue, interphase cells or metaphase chromosomes (Fig. 2) (Carpenter, 2001). FISH spawned comparative genomic hybridization (CGH) and spectral karyotyping (SKY), which represent more sophisticated applications of molecular cytogenetics (Kallioniemi et al., 1992; Schröck et al., 1996).

CGH is the comparison of two genomes by hybridization, and is intended to give researchers the ability to locate DNA copy number anomalies with greater frequency and increased sensitivity as compared to conventional cytogenetic methodologies. In CGH, researchers generate fluorescent probes from normal and tumor genomes, and simultaneously hybridize these probes to normal metaphase spreads (Thompson and Gray, 1993; Ried et al., 1997). Comparison of the relative fluorescence along each chromosome results in a ratio profile that shows chromosomal regions of relative gain (e.g., amplification) and loss (e.g., deletion) in the tumor genome. This is predicated upon the normal genomic probe binding uniformly to the normal chromosomes, whereas the tumor genomic probe will show an excess or deficit of specific chromosomes or portions of chromosomes, thereby revealing gains and losses of chromosomal material for the tumor.

The development of CGH preceded SKY, which is in essence a genomic scan using 24-color FISH (Schröck et al., 1996; Macville et al., 1997; Schröck et al., 1997). In SKY, each of the 22 autosomes and the 2 sex chromosomes are assigned a unique combination of fluorochromes, which allows for identification of all 24 of the chromosomes in a single hybridization. The development of ratio-labeling and combinatorial labeling schemes, in particular the inclusion of haptens, allowed for the number of differentially-labeled chromosome paints to exceed the number of 

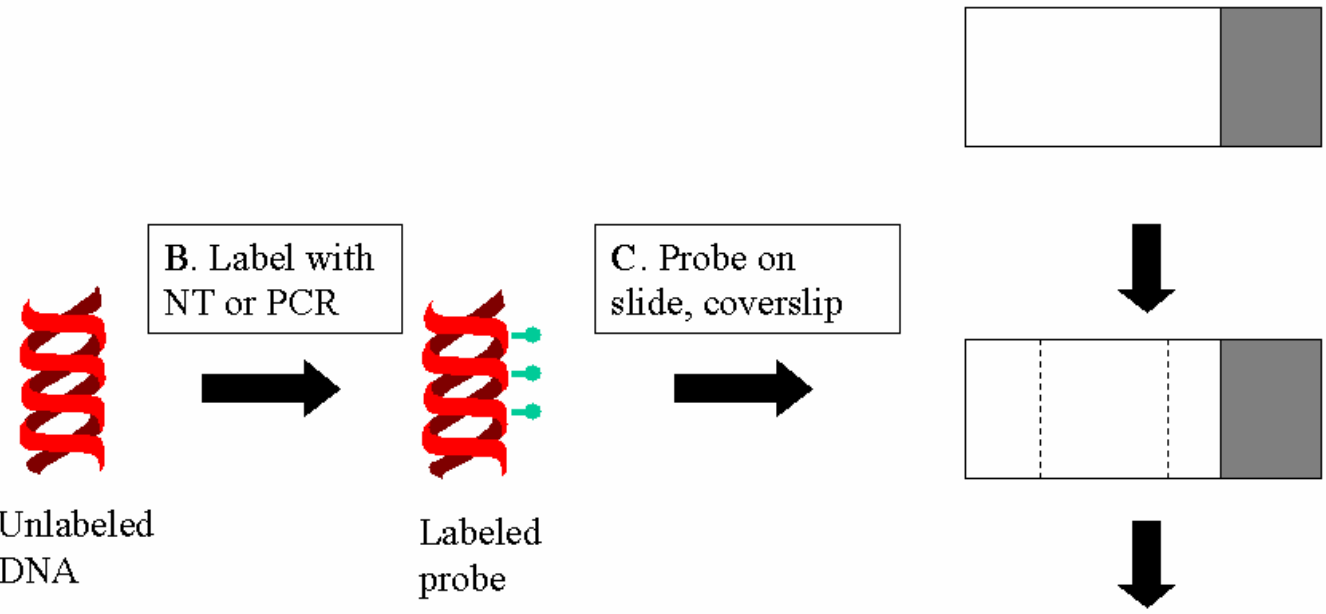

D. Simultaneous

F. Wash excess probe,

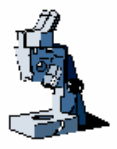
antibody detection

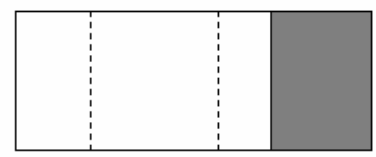
denaturation @ $75^{\circ} \mathrm{C}$

G. Fluorescence visualization

E. Hybridize overnight @ $37^{\circ} \mathrm{C}$

Figure 2. Basic FISH Protocol. This schematic outlines the critical steps in FISH. (A) The first step is to prepare metaphase spreads or interphase nuclei on microscope slides, and age these slides for at least a week. Pretreatment of the slide may or may not be necessary. (B) Probe is generated via nick translation or polymerase chain reaction. (C) This probe is placed on the slide and coverslipped. (D) The slide and probe are simultaneously denatured at or near $75^{\circ} \mathrm{C}$ in order for hybridized probe and target DNA to anneal. (E) The hybridized slide is incubated overnight at or near $37^{\circ} \mathrm{C}$. (F) On the following day, a post-hybridization wash removes excess unbound probe from the slide, and the probe is detected using antibody layering schemes as needed. (G) A DNA counterstain is applied prior to mounting of the slide in antifade solution for visualization with fluorescence microscopy. 
fluorochromes (Garini et al., 1996; Schröck et al., 1997; Tonon et al., 2000; Schröck and Padilla-Nash, 2000). Although the human eye can not resolve the subtle color differences between some fluorochromes, a Fourier transformation of emitted fluorescence gives each chromosome a distinct spectrum. Construction of the spectral karyotype elucidates both numerical and structural, anomalies. This allows determination of an accurate karyotype of many tumor metaphase spreads, which often contain both subtle and complex chromosome rearrangements. An additional feature of SKY is that chromosomal material that can not be karyotyped with confidence in a classical cytogenetic analysis (such as marker chromosomes) can be correctly identified.

Uveal melanoma is but one of a myriad of cancers afflicting humans today. This particular solid tumor is the primary intraocular tumor manifested in adults, and results in loss of vision and in many cases death. Uveal melanoma is any malignant melanocytic tumor originating in the pigmented vascular layer of the eye including the iris, ciliary body and choroid (Fig. 3). Epidemiologic studies show that those at greatest risk are individuals with lightly pigmented skin and/or lightly pigmented eyes (McLean and Gamel, 1996; Regan et al., 1999). The most frequent chromosomal abnormalities are found on chromosomes 3, 6, and 8; other chromosomes have been implicated with varying consistency (Speicher et al., 1994; Prescher et al., 1996; White et al., 1998a). The tumor is thought to share commonalties with other melanomas, yet maintain its own defined subset of causal aberrations (Griffin et al., 1988).

In order to ascertain the chromosomal aberrations that contribute to uveal melanoma, we pursued 2 avenues of research. The first was to employ CGH, SKY and 


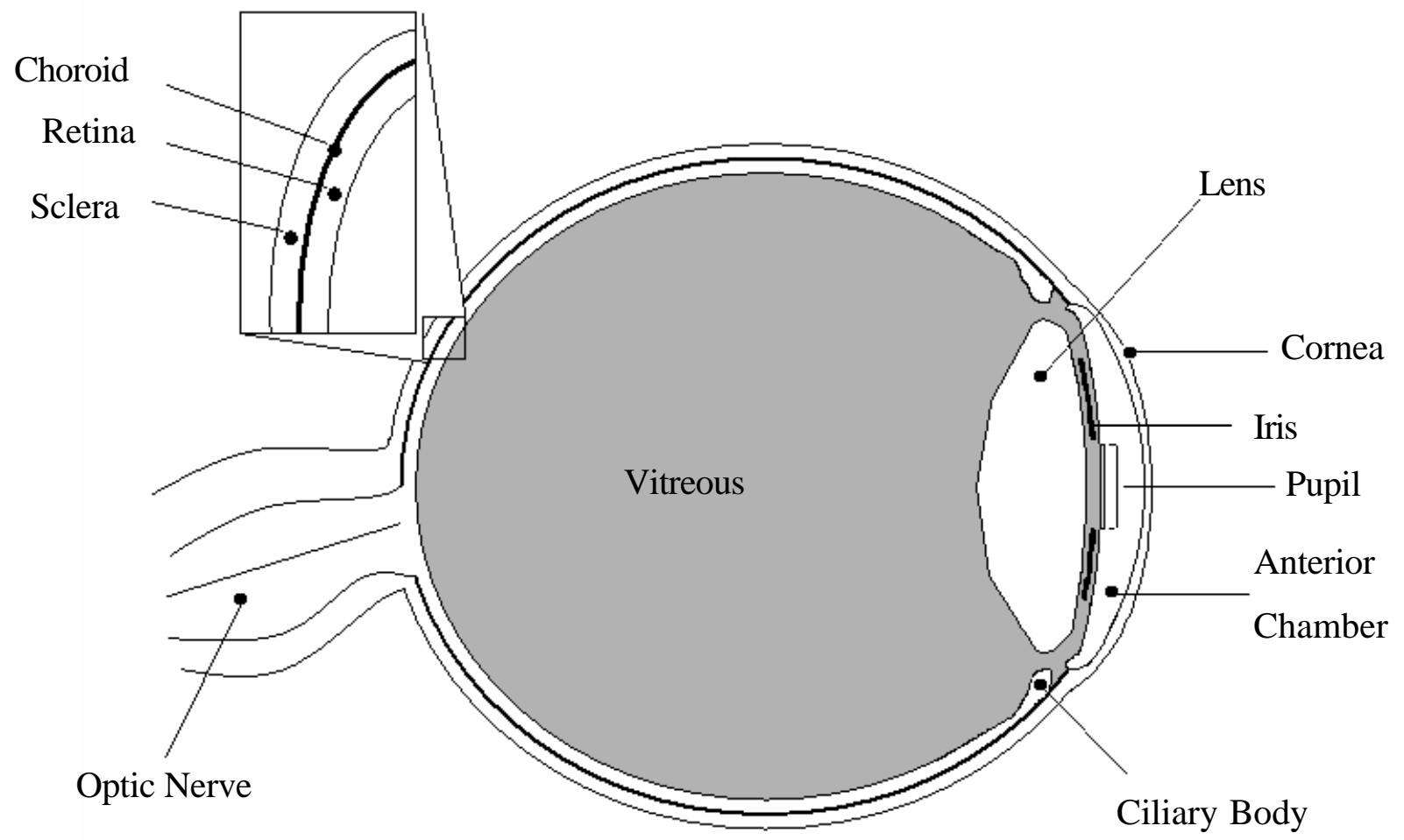

Figure 3. Anatomy of the Ocular Globe. This schematic identifies landmarks within the human eye, and orients the reader with regard to the regions of origin for uveal melanoma. These regions include the choroid, ciliary body and the iris. 
FISH to provide a complete molecular cytogenetic evaluation of 10 uveal melanoma cell lines. The second was a comprehensive CGH analysis of 100 archival formalin-fixed paraffin-embedded (FFPE) uveal melanoma tissue samples.

In the first portion of our study, we characterized 10 uveal melanoma cell lines (Table 1) by CGH, SKY and FISH to identify chromosome abnormalities. Characterization of cell lines is often employed as an initial step in characterization and classification of a disease, as they model the alterations in actual tumors. Uveal melanoma cell lines are typically derived from enucleated tissue. Enucleation, or removal of the entire globe of the eye, is a common treatment strategy. Cells from enucleated tissue can be transformed, cultured in an appropriate medium, and maintained as immortal cell lines for subsequent characterizations (De Waard-Siebinga et al., 1995; Luyten et al., 1996). Previous characterizations of uveal melanoma cell lines included not only classical cytogenetic analysis, but also evaluations of specific genes and their expression (Blom et al., 1997; Luyten et al., 1998; Chana et al., 1999). Uveal melanoma cell lines have also been used to model the effects of developing or established treatments (Logani et al., 1995a and b). We expected that a combination of CGH, SKY and FISH would increase sensitivity for detection of abnormalities, and reveal chromosomal regions applicable to uveal melanoma diagnosis, prognosis and treatment. We also expected that the combination of these 3 techniques would ascertain the prevalence of monosomy 3, as it is a hallmark of uveal melanoma (Speicher et al., 1994; Prescher et al., 1996; White et al., 1998a).

For the second portion of our study, we analyzed 100 archival FFPE uveal melanoma cases by CGH. These cases were obtained from the Armed Forces Institute of 
Table 1. Uveal Melanoma Cell Lines Used in this Study

\begin{tabular}{|c|c|c|c|c|c|c|}
\hline Code & Cell Line & Tumor & Origin $^{a}$ & $\operatorname{Sex}^{\mathrm{a}}$ & Media & Reference $^{b}$ \\
\hline 1 & $92-1$ & primary & choroid & $\mathrm{F}$ & RPMI 1640 & De Waard-Siebinga et al., 1995 \\
\hline 2 & MEL270 & primary & ciliary body & M & $"$ & Chen et al., 1997 \\
\hline 3 & MEL285 & primary & & $\mathrm{F}$ & $"$ & \\
\hline 4 & MEL290 & primary & & $\mathrm{F}$ & $"$ & \\
\hline 5 & OCM-1 & primary & choroid & $\mathrm{F}$ & Ham's F-12 & Kan-Mitchell et al., 1989 \\
\hline 6 & OCM-3 & primary & & & $"$ & \\
\hline 7 & OCM-8 & primary & & & $"$ & \\
\hline 8 & OM431 & primary & choroid & & $"$ & Albert et al., 1984 \\
\hline 9 & OMM-1 & metastatic & $\begin{array}{l}\text { subcutaneous metastatic } \\
\text { lesion post-enucleation }\end{array}$ & M & DMEM & Luyten et al., 1996 \\
\hline 10 & OMM-2.3 & metastatic & $\begin{array}{l}\text { liver metastisis of ciliary } \\
\text { body tumor (MEL270) }\end{array}$ & M & RPMI 1640 & \\
\hline
\end{tabular}

${ }^{\mathrm{a}}$ Blank entries reflect data unavailable at the time of the study.

${ }^{b}$ All cell lines provided courtesy of Dr. Jerry Y. Niedercorn and Ms. Elizabeth Mayhew, both at the Department of Ophthalmology at the University of Texas Southwestern Medical Center at Dallas, in Dallas, TX. Those that have establishment papers in the published literature have been cited. 

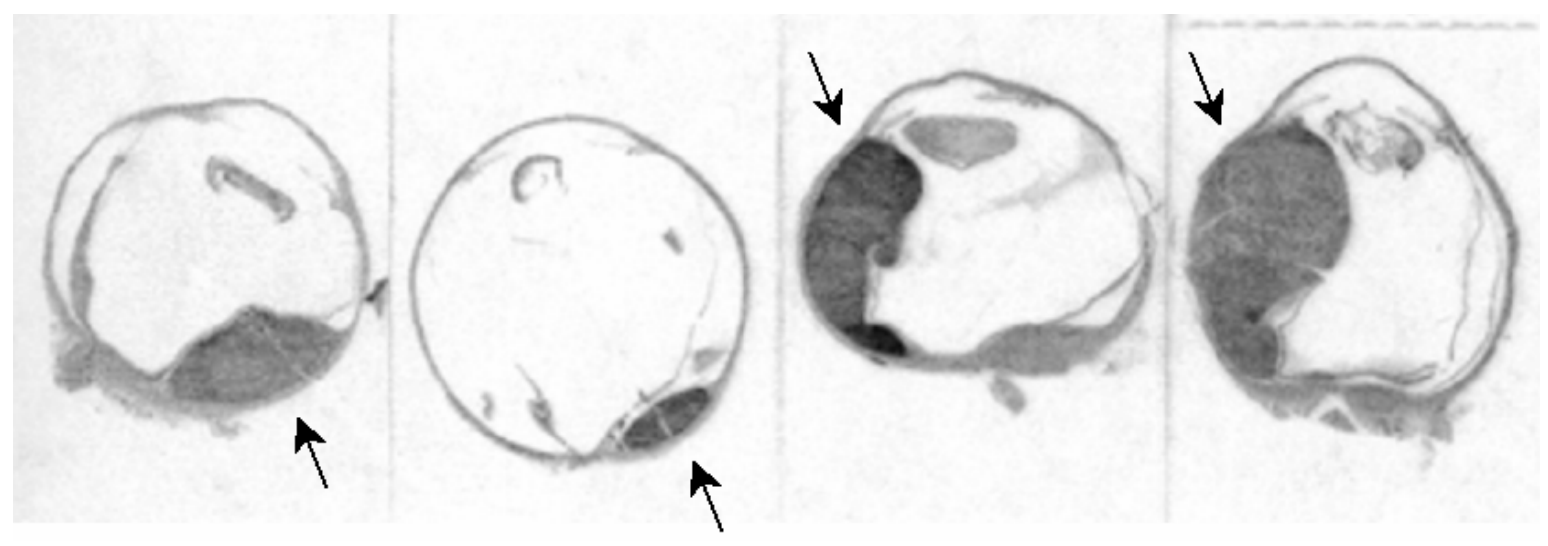

Figure 4. Examples of Uveal Melanoma Tumors. Four hematoxylin and eosin (H\&E) stained archival FFPE tissue sections from different uveal melanoma tumor cases. The darkest mass in each ocular globe (indicated by arrows) is the uveal melanoma tumor. 
Pathology (AFIP) tissue archives, and comprised of 2 slide-mounted $6 \mu \mathrm{m}$ sections of archival FFPE tumor tissue from enucleated human eyes (Fig. 4). Utilization of archival tissue allows for analysis of a larger number of cases, particularly when studying a tumor of low incidence that yields only a few fresh tissue samples each year. Also, archival FFPE tissue allows for correlation of experimental findings with comprehensive patient follow-up, such as visible manifestations of the disease, tumor characterization and grading, patient progress after tumor excision, and survival. Of the 100 cases, 51 survived greater than 9 years without detectable metastasis, and the remaining 49 cases succumbed to metastatic disease within 9 years of diagnosis. It was expected that the regions of amplification and deletion seen in the samples from each patient would be homologous to those seen in the cell line study, and would correlate with survival to allow determination of prognostic factors associated with uveal melanoma. Tumor progression is thought of as a stepwise accumulation of abnormalities in critical genes, and it was our expectation that some chromosomal regions of gain and loss would correlate with malignancy and progression of uveal melanoma (Forozan et al., 1997). Anomalies were tested for univariate prognostic significance, and the most powerful predictors were compared with other histological or clinical predictors in a multivariate analysis.

The amalgamation of results from the cell line and archival tumor portions of our study yielded extensive insight into the chromosome dosage abnormalities characteristic of uveal malignant melanoma. Further, the synthesis of our results allowed us to identify not only predictive loci for this tumor, but also to make some speculation about the genes 
involved in development and/or progression of this tumor. Our ultimate goal was to direct further ge ne-specific study of malignancy in uveal melanoma. 


\section{LITERATURE REVIEW}

\section{Cytogenetics.}

The foremost discovery in genetics was the elucidation of the double-helical structure of DNA by Watson, Crick, Wilkins and Franklin by X-ray diffraction (Watson and Crick, 1953). At around the same time, T. C. Hsu first described the use of a hypotonic gradient to swell a cell, allowing for a closer glimpse of the components within the cell membrane (Fig. 1) (Hsu, 1952). In his initial work, Hsu found 23 pairs of autosomes and a pair of sex chromosomes (Fig. 5) (Hsu, 1952). By adapting Hsu's technique, Tjio and Levan revised Hsu's findings of 48 chromosomes, and instead found that humans have a 46 chromosome complement (Tjio and Levan, 1956). A few years later, Hungerford and colleagues produced the first karyotype from peripheral blood culture (Hungerford et al., 1959).

Chromosome banding was developed by Casperson and colleagues in 1968, providing a useful technique that allowed the entire genome to be analyzed at once (Casperson et al., 1968; Pinkel et al., 1986a and b; du Manoir et al., 1993). Chromosome banding analyses are of particular import in cancer research, as chromosomal abnormalities can be linked with a cancer phenotype, and identification of consistent or recurrent chromosomal aberrations in tumors is a useful step toward identifying genes involved in the development or progression of the disease (Macville et al., 1997). However, analyses of solid tumors are complicated by low mitotic indices and low quality chromosomal banding, making identification of complex chromosomal aberrations immensely difficult by banding alone (Macville et al., 1997; Veldman et al., 1997; Ried et al., 1997; Rothmann et al., 1998). Hence, there is a critical need 


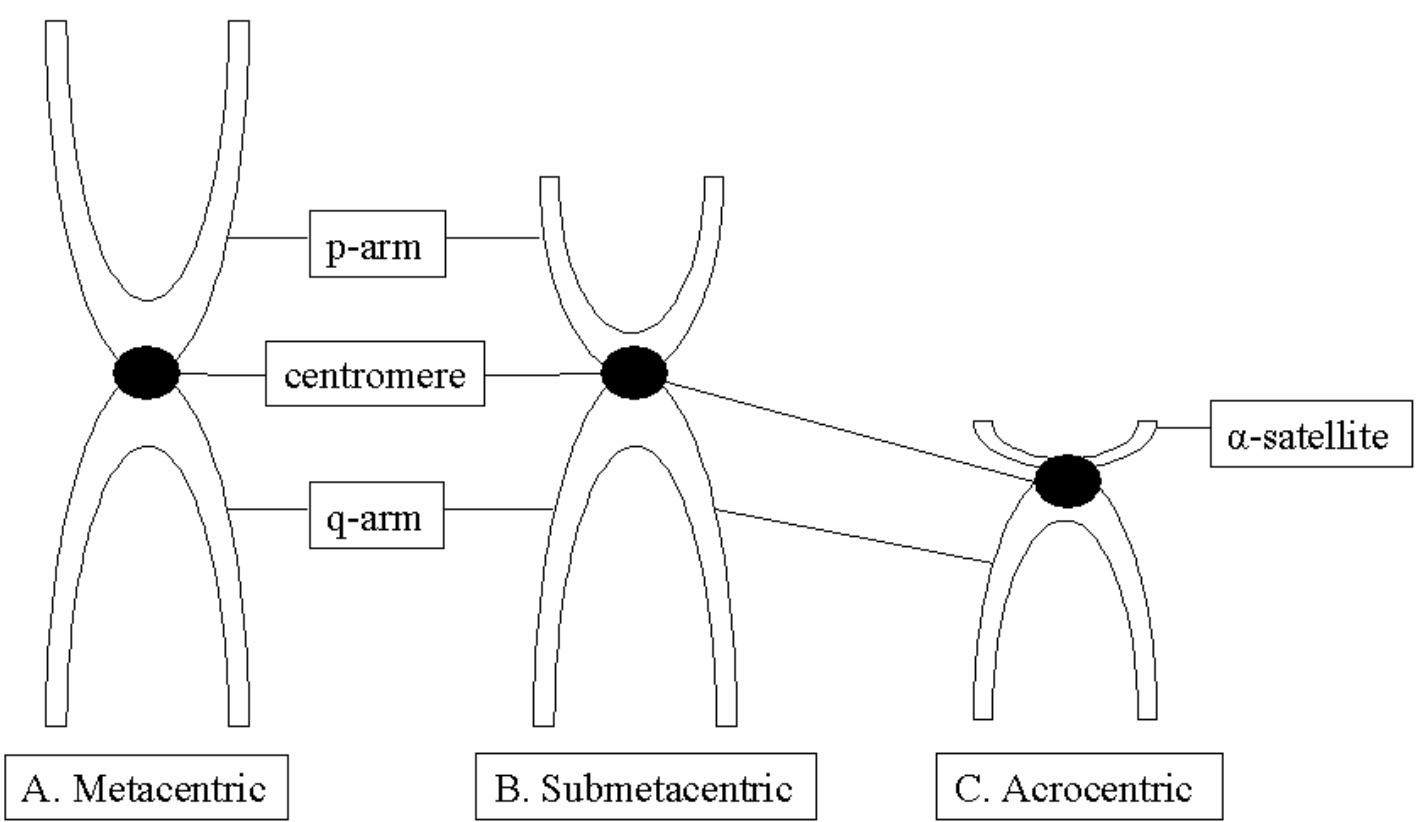

Figure 5. Anatomy of the Chromosomes. Chromosomes are comprised of 2 arms, the p-and the q-arm, and the arms are connected by a centromere. The exceptions are the acrocentric chromosomes, where the p-arm is actually alpha-satellite repeats. Chromosomes can be broken into 3 groups based on centromere location, and irrespective of size or relative DNA content. (A) The metacentric chromosomes are defined by their centrally-located centromere, and include 1, 2, 3, 16, 19 and 20. (B) The submetacentric chromosomes have an offset centromere that makes the p-arm shorter than the q-arm. This group includes 4, 5, 6, 7, 8, 9, 10, 11, 12, 17, 18 and X. (C) The acrocentric chromosomes are predominantly comprised of q-arm material, but have small satellites above the centromere, and include 13, 14, 15, 21, 22 and Y. 
to develop more accurate and reliable methods for determination of chromosome abnormalities.

\section{FLUORESCENCE IN SITU HYBRIDIZATION.}

In situ hybridization (ISH) was developed as a means of detecting nucleic acid sequences in morphologically intact samples (Gall and Pardue, 1969; John et al., 1969). It is based on the principle that DNA is able to denature and renature in a complementary fashion (Fig. 6). In principle, two nucleic acid sequences are found to be complementary; (1) the probe, which is the isolated and tagged sequence that the researcher is interested in localizing, and (2) the target sequence, which is a length of nucleotides within the DNA of the sample that matches the probe sequence. When both the sample and probe are denatured together, and allowed to reanneal, the probe binds to its complementary sequence in the target DNA. After the DNA has reannealed, the probe can be visualized and its location pinpointed within the sample.

The first applications of ISH were in 1969, when Gall and Pardue hybridized radioisotope-labeled ribosomal ribonucleic acid (rRNA) to tissue squashes, revealing the complementary ribosomal deoxyribonucleic acid (rDNA) in situ (Gall and Pardue, 1969). Also in 1969, John and colleagues experimented with RNA-DNA hybrids visualized by autoradiography (John et al., 1969). In the earliest applications of ISH, the nucleic acid probe (either DNA or RNA) was labeled with a radioisotope, hybridized to its complementary DNA sequence in a cell or tissue sample, and visualized using autoradiography (Gall and Pardue, 1969; John et al., 1969). The first non-isotopic ISH

application involved immunofluorescence detection of the RNA-DNA hybrids by a 

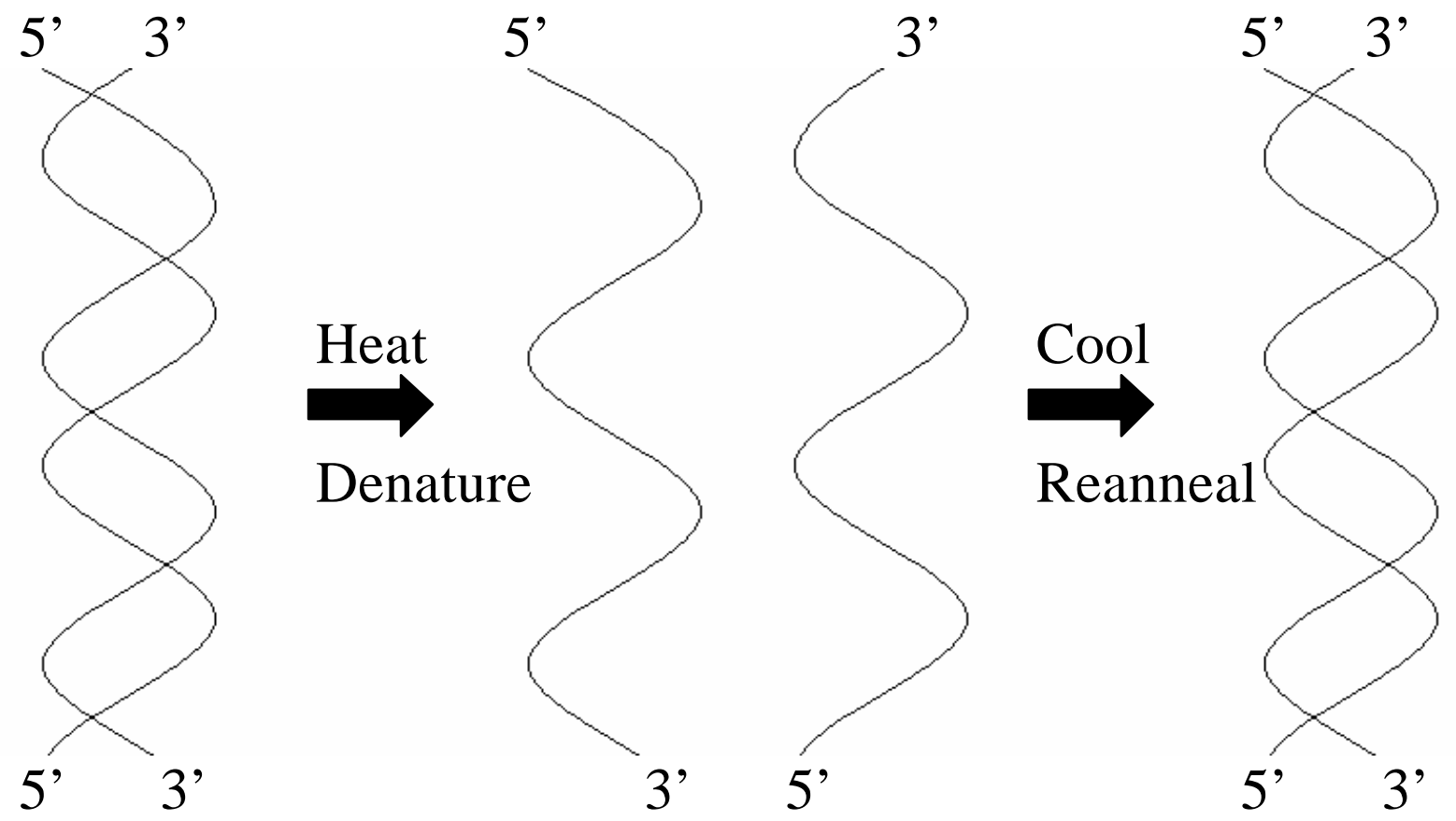

Figure 6. Denaturation/Renaturation of DNA. DNA will denature when the ambient temperature is sufficiently high, and will reanneal when the temperature falls below the melting point. It is this property of DNA that makes hybridization feasible. In a basic hybridization, the probe will find its complementary sequence of nucleotides in the target DNA and bind to them, which functionally incorporates the probe into the target DNA once all of the DNA has had an opportunity to reanneal. Then, the probe can be visualized and localized within the target DNA. This simple property of DNA forms the basis for such techniques as ISH, FISH, CGH and SKY.

This figure was adapted from University of Michigan Health System, http://seqcore.brcf.med.umich.edu/doc/educ/dnapr/pg2.html. 
fluorescent antibody bound to the hybrids (Rudkin and Stollar, 1977). Use of radioisotope labeling was superceded by applications of non-isotopic conjugated probes in 1980s (Bauman et al., 1980; Langer et al., 1981; Pinkel et al., 1986b). Bauman and colleagues described the covalent linkage of tetramethyl rhodamine isothiocyanate (TRITC) to RNA, which when used simultaneously with a ${ }^{3} \mathrm{H}$-labeled complementary RNA (cRNA) allowed both fluorescence and scintillation quantitation (Bauman et al., 1980). Technically, this was the first application of a directly-labeled fluorescent probe.

FISH completed its evolution from ISH, by replacing all radioisotopes with fluorescence labeling (Fig. 2). The first alternatives to labeling with radioisotopes were biotin-labeled UTP and dUTP, described by Langer and colleagues in 1981 (Langer et al., 1981). The use of non-isotopic probes has lead to improved resolution, decreased preparation time and increased probe stability (Trask, 1991).

In 1986, Pinkel and colleagues described the first fluorescent hybridizations employing biotinylated probes and fluorescently-labeled avidin (Pinkel et al., 1986a and b). Indirectly-labeled probes are conjugated with a reporter-binding molecule (or hapten), whereas directly-labeled probes are conjugated with a fluorochrome (Joos et al., 1994) (Fig. 7). Haptens include biotin, digoxigenin, dinitrophenyl (DNP), aminoacetylfluorene (AAF), mercury and sulfonate (Trask, 1991; Luke and Shepelsky, 1998). Biotin is detected with either a fluorochrome-conjugated avidin or an anti-biotin antibody, while mercury is detectable with a ligand bearing a sulfhydryl group (Trask, 1991). Digoxigenin and the remaining haptens are detected with fluorescently-labeled antibodies (Trask, 1991). The use of directly-labeled probes is a more time-efficient method, and generally results in less background than indirectly-labeled probe 
A.

Fluorochrome conjugated dUTP

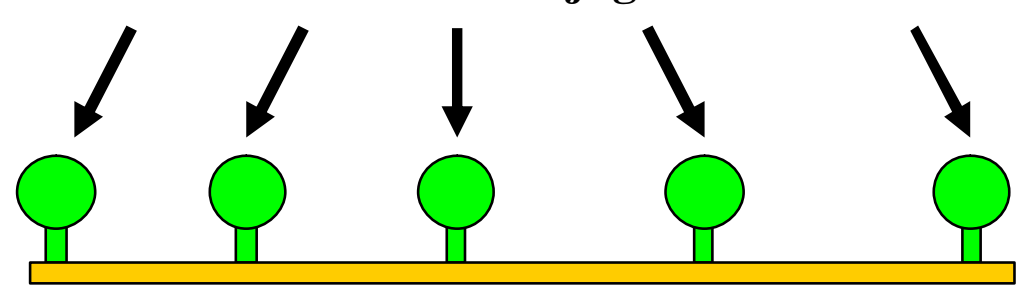

U A C U A G U C A G U G C G U

B.

Avidin reporter fluorochrome

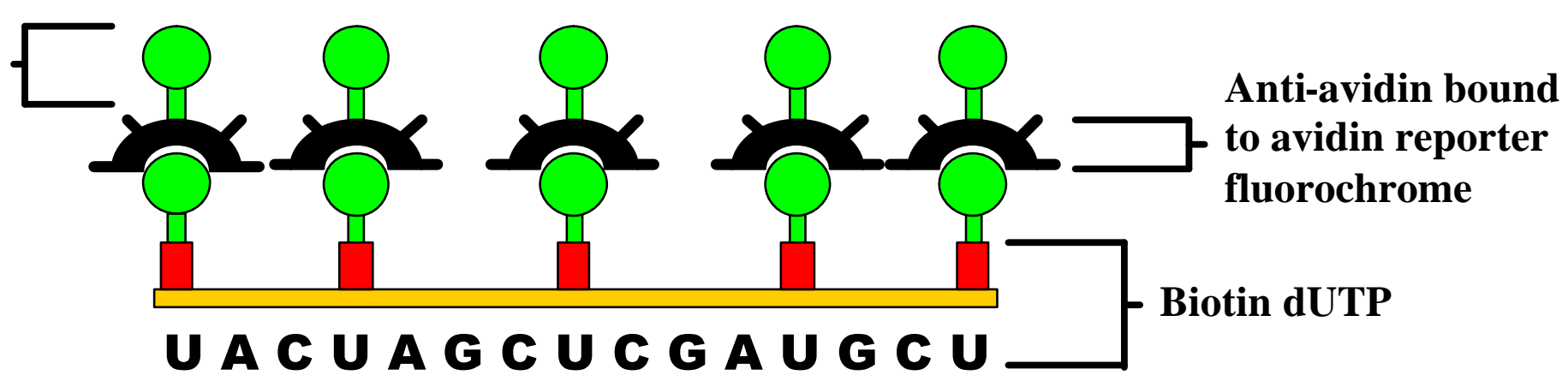

Figure 7. Comparison of Directly- and Indirectly-labeled Probes. (A) Directly-labeled probe. The reporter fluorochrome is directly conjugated to the dUTP, and visualization of the probe is possible immediately following hybridization. (B) The indirectly-labeled probe has a hapten-labeled dUTP incorporated into its sequence, in this case biotin-dUTP, and detection using antibody layering is required following hybridization. An avidin reporter fluorochrome binds to each biotin-dUTP, and a biotinylated anti-avidin binds to each avidin. A second avidin reporter fluorochrome then binds to the biotinylated anti-avidin. The antibody layering process is repeated as needed to amplify the probe signal by successive addition of avidin reporter fluorochrome layers.

This figure courtesy of Dr. Alison E. Director-Myska, AFIP. 
applications upon visualization. The advantage of direct incorporation of the fluorochrome into the probe, is that the fluorochrome is confined to the binding site. In indirect applications, the dyes precipitate around the binding site and are therefore ess specific and result in increased background noise (Demetrick, 1999). However, increased sensitivity is achieved through the use of an indirectly-labeled probe, which allows for multiple layers of antibodies, thus increasing the signal intensity.

The advent of FISH narrowed the gap between cytogenetics and molecular genetics, and gave rise to the field of molecular cytogenetics (Macville et al., 1997). FISH, as used in molecular cytogenetics, is equally applicable to metaphase spreads, interphase nuclei preparations, and tissue sections (Cremer et al., 1986; Lichter et al., 1988a; Devilee et al., 1988; Eastmond and Pinkel, 1989; Trask, 1991; Joos et al., 1994). "Interphase cytogenetics" was a term coined by Cremer and colleagues in 1986 for the detection of chromosome aberrations in interphase cells (Cremer et al., 1986). They later expanded this definition to include detection in non-mitotic cells at any stage of cell division, due to modifications made by Lichter et al., (Cremer et al., 1988; Lichter et al., 1988a).

Lichter and colleagues refined suppression of non-informative regions of repeat, found in regions such as centromeres, telomeres, heterochromatin, and other families of repeat sequences in the genome, thus enhancing detection of chromosome-specific regions of repeat (Lichter et al., 1988a). "Chromosomal in situ suppression" (CISS), a term first coined in 1988, described the use of unlabeled competitor DNA to suppress of regions of repeat (Lichter et al., 1988a). These regions of repeat are common on many chromosomes in the genome, and also are very quick to reassociate after denaturation 
(Lichter et al., 1998a and b). Both of these factors interfere with accurate hybridization between the probe and target DNA. Incorporation of human Cot-1 DNA inhibits the binding of repetitive sequences such as the Alu I and Kpn I repeat families (Lichter et al., 1988a and b). Incorporation of salmon testes DNA binds repeat sequences other than the two previously mentioned families of repeats, and prevents non-specific binding of the probe to the slide or coverslip (Lichter et al., 1988a). Each of these are included in the probe cocktail for suppressive hybridization schemes.

The most important reason for development of interphase FISH techniques, is that it alleviates the need for metaphase preparations, which are often limited or otherwise unavailable in tumor preparations (Bayani and Squire, 2002). The use of interphase nuclei provides higher resolution due to the decreased condensation of nuclear DNA (Lawrence et al., 1988; Devilee et al., 1988; Joos et al., 1994).

Once the ability to detect a single probe in interphase applications had been refined, the detection of mulitple targets was the next logical step. A double-labeled single-target FISH experiment by Cremer and colleagues marked the first multicolor fluorescence FISH experiment, and a double-labeled double-target FISH hybridization was carried out in solid tumors shortly thereafter (Cremer et al., 1988; Hopman et al., 1988). These experiments further demonstrated the increased resolution in interphase cells as compared to metaphase preparations (Hopman et al., 1988). In 1989, Nederlof and colleagues were able to detect 3 hybridization targets using a third fluorochrome spectrally distinct from fluorescein isothiocyanate (FITC) and TRITC (Nederlof et al., 1989). In 1990, the same group expanded the number of hybridization sites to 4 using the first combinatorial labeling schemes (involving 2 fluorochromes) (Nederlof et al., 1990). 
At that time, it was postulated that the number of spectrally-distinct fluorescent hybridization sites could be expanded to 7 using a combination of fluorochromes and conjugated haptens. The complex hybridization of 7 probes to 7 loci was not realized until 2 years later (Ried et al., 1992a).

Ried and colleagues were also able to utilize 5 distinct whole chromosome paints in a single hybridization to assay ploidy of 5 chromosomes critical in prenatal diagnosis (Ried et al., 1992b). Also in 1992, Dauwerse et al. demonstrated that combining 2 fluorochromes in varying ratios (ratio-labeling) allowed for the distinction of up to 7 whole chromosome paints in a single exposure, significant because the maximum possible number of distinct loci was also 7 at that time (Dauwerse et al., 1992). They also suggested that inclusion of a third fluorochrome in their ratio-labeling scheme would allow distinction of half of the chromosome complement (Dauwerse et al., 1992). FISH was only limited by the ability to distinguish the spectra of multiple probes in a single preparation. In other words, the number of hybridization sites was only limited by the ability of the analysis software and the user to distinguish between different fluorochromes or combinations of fluorochromes (Joos et al., 1994; Garini et al., 1996). The evolution of multicolor FISH analyses would eventually lead to development of genome-labeling techniques, such as multiplex-FISH (M-FISH) and SKY, which allow distinction of all 22 autosomes and the sex chromosomes in a single hybridization.

Since its inception, FISH has evolved into a technique applicable to dividing or non-dividing cells in either fresh or archival tissue (Trask, 1991). FISH can be used as an exploratory or a confirmatory technique. Given an appropriate hybridization scheme, FISH can be employed to detect inversions, translocations, deletions, dicentrics and ring 
chromosomes (Trask, 1991). The technique itself is generally not very time consuming, as detection and/or visualization can often be performed a day or two after hybridization. Specificity of a probe depends on base-pair composition (G-C bonds are stronger than AT), temperature of denaturation and hybridization, as well as the salt and formamide concentrations in the hybridization mixture (McNicol and Farquharson, 1997).

There are essentially three types of probes currently widespread in use, each with a slightly different intended application. The first are locus-specific probes, which, hybridize to a specific chromosomal locus (Tonon et al., 2000). These probes are generated from plasmids, phages, cosmids, or YAC-clones (Cremer et al., 1986; Joos et al., 1994). The efficiency of the hybridization is proportional to the size of the probe, because the ability to detect a hybridization decreases as the probe size decreases (Trask, 1991). A gene-specific probe, one kind of locus-specific probe, is generated from an entire gene or a portion of a gene and hybridized to determine where it will localize (e.g. the location of the ge ne on a chromosome).

The second type of probe is a chromosome-specific repetitive sequence probe, and this type of probe can be targeted to centromeres (heterochromatin containing alphoid repeats or alpha-satellite DNA) or telomeres (highly repetitive sequences at the ends of each chromosome). These probes are typically used in chromosome enumeration studies, which are focused on determination of the number of copies of a given chromosome in a preparation (Pinkel et al., 1986b; Eastmond and Pinkel, 1989; Joos et al., 1994; McNicol and Farquharson, 1997; Demetrick, 1999). Hybridization of these probes is typically very intense, as the areas hybridized in conserved portions of heterochromatin are often very compact (Trask, 1991). 
The third type are whole chromosome probes, also known as whole chromosome paints, which are in actuality a group of probes all generated from a single chromosome that fluoresce along the length of the entire chromosome when hybridized (Pinkel et al., 1988). This type of FISH probe is used to identify chromosome-specific material and determine rearrangements associated with the probed chromosome in the genome (Tucker et al., 1994; Carter, 1994; Demetrick, 1999). These genomic rearrangements can be symmetrical (e.g. reciprocal translocations) or asymmetrical (e.g. insertions and nonreciprocal translocations) exchanges (Tucker et al., 1994). Initially, flow-sorted chromosomes were used to generate DNA libraries, which were later used to make whole chromosome paints (Carter, 1994; Joos et al., 1994; Luke and Shepelsky, 1998). Flowsorting remains a viable technique for isolating normal chromosomes for painting, and can also be used to isolate populations of aberrant chromosomes for production of painting probes provided the aberrant chromosomes are distinguishable from normal chromosomes using this technique. Alternatively, microdissection of specific chromosomes is possible and allows for aberrant chromosomes to be individually selected and then used to generate probes (Ried et al., 1997; Bayani and Squire, 2001). Combination of 24 chromosome-specific paints into a single hybridization cocktail is the essence of spectral karyotyping, while generation of single-fluorochrome whole genome probes for CGH applications is an expansion upon the use of whole chromosome paints. Even though combinatorial and ratio-labeling schemes increased the scope and application of FISH, it is cumbersome to screen tumor genomes for gains, losses, and rearrangements with individual FISH probes (Raap, 1995; Raap, 1998). 


\section{COMPARATIVE GENOMIC HYBRIDIZATION.}

In 1992, Kallioniemi et al. developed a molecular cytogenetic technique capable of detecting and mapping relative DNA sequence copy number differences between 2 genomes competitively hybridized to normal chromosomes (Kallioniemi et al., 1992). In this technique, a copy number karyotype is generated for a tumor by comparing the DNA from malignant and normal cells, thereby identifying regions of gain or loss in the DNA along the length of each chromosome. In actuality, the gains and losses are representative of global genetic variation in the test sample, and the data output is really a measure of genomic balance (Kallioniemi et al., 1992; Thompson and Gray, 1993; Houldsworth and Chaganti, 1994; Ried et al., 1997). The inception of CGH was directly linked to the need for a rapid and comprehensive assessment of genetic balance where DNA, not cells for culture and chromosome preparation, was the only available material (du Manoir et al., 1993; du Manoir et al., 1995b; Raap, 1995).

CGH is a quantitative two-color fluorescence in situ suppression hybridization technique useful in detecting copy number changes throughout an entire genome (Thompson and Gray, 1993; Ried et al., 1997). In other words, it is essentially doublelabeled double-target FISH, wherein total genomic tumor DNA and total genomic DNA from normal diploid tissue are differentially labeled and then simultaneously hybridized to normal metaphase chromosomes. The resulting copy number ratio reveals any genomic imbalance between the two. CGH is an improvement on single-color hybridizations, because the normal DNA is an "internal hybridization intensity control" for each location in the genome (Kallioniemi et al., 1994). 
CGH has numerous advantages, most notably it elucidates gains (e.g. amplifications) and losses (e.g. deletions) of DNA, often undetectable via classical cytogenetic techniques. Classical cytogenetic analyses are hindered by the inability to produce large numbers of high quality tumor metaphases due to low mitotic indices, poor growth in culture, and the increased likelihood of bacterial and/or necrotic tissue contamination (Joos et al., 1994; Houldsworth and Chaganti, 1994; Isola et al., 1994). In $\mathrm{CGH}$, extracted genomic DNA is the only material required from the test sample, increasing its application to include both fresh and archival tissue (Houldsworth and Chaganti, 1994; Ghazvini et al., 1996). Karyotypic analysis of solid tumors is difficult due to the instability of tumor cells in culture, but $\mathrm{CGH}$ alleviates the need for in vitro tumor cell culture, because, in contrast to traditional karyotyping, it only requires DNA and therefore reduces subpopulation selection (Joos et al., 1994). Imbalances in a subset of the cells in a given sample do not interfere with the global analysis of the tumor as long as tumor cells account for most of the DNA (du Manoir et al., 1993; Lundsteen et al., 1995). However, the effect of intratumor genetic heterogeneity on sensitivity of CGH is variable, as heterogeneous aberrations in a given sample are averaged while homogeneous aberrations are emphasized (Kallioniemi et al., 1992; Kallioniemi et al., 1993). Similarly, if normal tissue accounts for more than $50 \%$ of the total DNA from the test sample, ratio changes may not be reliably detected (Kallioniemi et al., 1994).

As mentioned earlier, one advantage of $\mathrm{CGH}$ is the construction of DNA probes from archival FFPE tissue; this is unique in that the vast majority of cytogenetic techniques require viable tissue (Isola et al., 1994; du Manoir et al., 1995b). Utilization of FFPE tissue allows for analysis of a larger number of cases and for correlation of 
experimental findings with comprehensive patient follow-up and survival data. FFPE tumor analysis allows for investigation of very old samples, however, fixation methods may negatively affect DNA quality and yield (Isola et al., 1994). In analysis of FFPE tumors, microdissection of tissue sections, using hematoxylin and eosin ( $\mathrm{H} \& \mathrm{E})$ stained slides for confirmation, has both advantages and disadvantages. An obvious advantage is the selection of only tumor material, because, as mentioned earlier, normal cells "dilute" the CGH results (du Manoir et al., 1993; Houldsworth and Chaganti, 1994; Joos et al., 1994). The disadvantages of tumor microdissection lie in the small yield of DNA, particularly from tissue of reduced cellular density such as the eye. This particular disadvantage can be overcome by polymerase chain reaction (PCR) amplification of relatively pure DNA. The use of degenerate oligonucleotide primed-PCR (DOP-PCR) for small quantities of DNA (as little as 100pg), as first described by Telenius and colleagues, ensures reliable amplification of the template DNA (Fig. 8) (Telenius et al., 1992a and b; Speicher et al., 1993; Kallioniemi et al., 1994; Joos et al., 1994). The DOP primer is actually a family of primers that have a degenerate middle sequence that allows the primers to bind to more sites within the template DNA, and thereby results in a probe that more accurately represents the original genomic DNA from the microdissected tumor.

The primary shortcoming of $\mathrm{CGH}$, is that it does not detect balanced rearrangements, such as translocations, because there are no copy number gains or losses associated with such events. For this same reason, point mutations and small intragenic rearrangements go undetected as well (Kallioniemi et al., 1994; Raap, 1995; Ried et al., 1997). CGH provides no insight regarding the arrangement of chromosome segments 


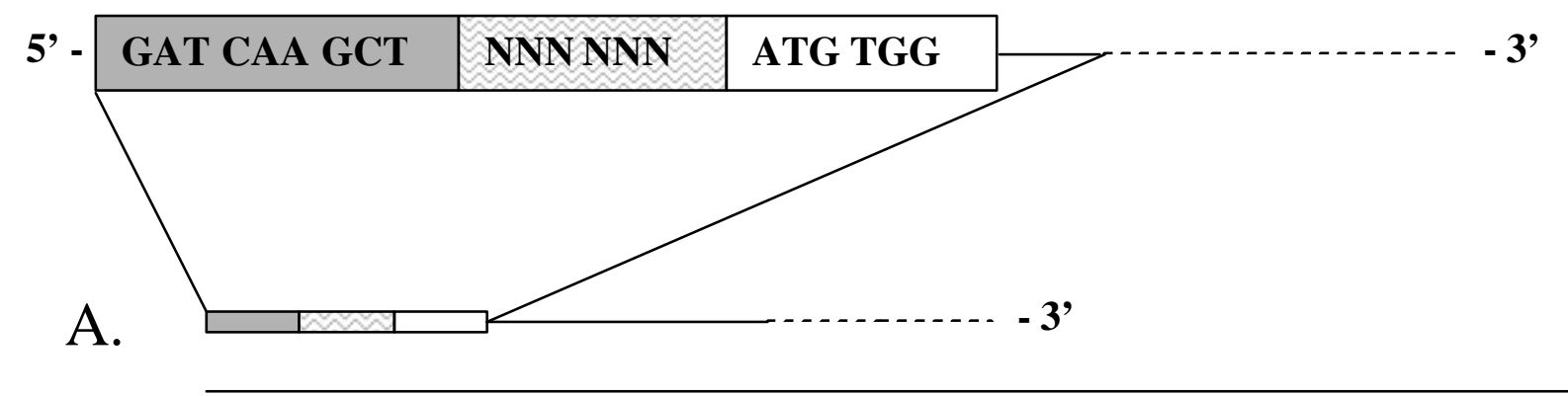

B.

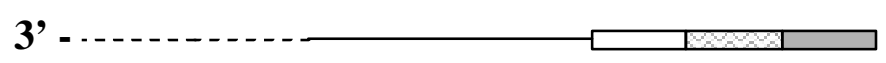

C.
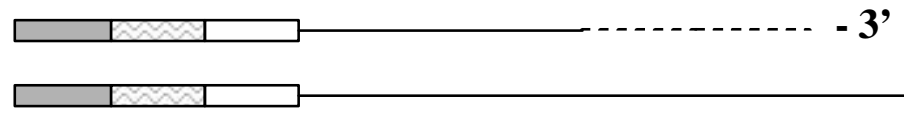

Figure 8. DOP-PCR Amplification of Genomic DNA. The 5' end sequence (gray) is more conserved than the 3' end of the primer (white). In between, is a variable six nucleotide sequence that introduces reduced stringency in to the PCR reaction. (A) In the first PCR cycle, the DOP primer binds to genomic DNA, and the inherent degeneracy of the primer combined with the reduced stringency of a relatively low annealing temperature allows it to bind with diminished specificity as compared to conventional PCR primers. The polymerase extends the sequence in the 3' direction from the sequence of primer attachment. (B) In the cycles that follow, the annealing temperature is still sufficiently low to allow relatively uniform amplification of all genomic sequences. (C) In the subsequent cycles, the annealing temperature is increased with each cycle, eventually requiring that the entire length of the primer binds to the template strand.

This figure was adapted from Telenius et al., 1992a. 
involved in reported gains and losses, nor does it help explain the origin of marker chromosomes. Further, the technique has no application for instances of clonal heterogeneity, since it only detects aberrations present in a majority of cells in a given sample (du Manoir et al., 1993; Raap, 1995; Ried et al., 1997; Chang and Mark, 1997). In such instances, abnormalities may cancel one another or be too infrequent to detect (Kallioniemi et al., 1994). Since CGH only detects copy number changes relative to the average copy number it can not distinguish genomic changes in ploidy, as there is no difference in the test:reference ratio between or within specific chromosomes in these cases (Kallioniemi et al., 1994). Sensitivity of detection for deletions is related to ploidy level as well; it is easier to detect a single copy loss in a diploid test sample than in a triploid or tetraploid test sample (Kallioniemi et al., 1994). Copy number changes are also difficult to quantify, even if they are not the result of aneuploidy, as the fluorescent signal tends to spread over a region greater than the length of the amplicon. This presumably results from the complex organization of the target DNA (Kallioniemi et al., 1992). An additional limitation is that some chromosomal aberrations will not be detected because they occur in regions smaller than can be resolved by CGH, which has a resolution of approximately 10-20Mb (Kallioniemi et al., 1992; Kallioniemi et al., 1993, Thompson and Gray, 1993). Therefore, such regions are only detected when the level of amplification is high (5-10 fold for $1 \mathrm{Mb}$ region) (Thompson and Gray, 1993; Houldsworth and Chaganti, 1994; Raap, 1995; du Manoir 1995b; Forozan et al., 1997). CGH is predicated upon appropriate chromosome classification of the normal target metaphase, whether by the software, the user, or an interaction between the two. Chromosome classification is an inherent source of error, as misclassification seriously 
affects the results, and it is also the greatest limiting factor in the processing of $\mathrm{CGH}$ data (Raap, 1995, Lundsteen et al., 1995).

Image preparation is of paramount importance in CGH. Homogeneity in an image is usually recognizable given a uniform reference paint. Homogeneity is highest when hybridizing a directly-labeled probe, reduced with indirectly-labeled probe, and reduced again with multiple layers associated with signal amplification of an indirectlylabeled probe (Lundsteen, 1995). Reduced homogeneity is akin to increased granularity or improperly sized probe, and indicative of a poor hybridization. The spread of the metaphase chromosomes, image realignment due to pixel shift between filter changes, and proper plane of focus all merit consideration in the processing of CGH images (du Manoir et al., 1995a). Selected metaphases should be of a similar degree of condensation, but some $\mathrm{CGH}$ analysis packages compensate for variations in condensation by normalization through linear stretching to fit the profile (Lundsteen, 1995; du Manoir et al., 1995b). Criteria for a good metaphase for analysis are as follows: (a) hybridization is smooth and high in intensity; (b) test (green) and reference (red) distributions are similar among sister chromatids; (c) background is low and uniform; (d) binding at centromeres and other regions of repeat is minimal; and (e) chromosomes are of acceptable morphology providing for adequate 4',6-diamidino-2-phenylindole (DAPI) banding and there are few or no overlaps (Kallioniemi et al., 1994). A minimum of 5 to 10 examples of each chromosome should be measured. In our lab, the preference was for 10 metaphases, which should yield 20 measurable examples of each chromosome. It should be noted that analysis of many chromosomes is not a substitute for quality hybridizations (du Manoir et al., 1995a and b). 
Fundamentally, CGH results are a comparison of green to red fluorescence intensities. These intensities are integrated along the length of each chromosome for both chromatids, and the result is the green:red ratio profile. Copy number gains and losses are determined by assigning absolute thresholds to the ratio profiles. Thresholds are determined by desired sensitivity, and may be adjusted to compensate for ploidy if a determination using independent methods is possible (du Manoir et al., 1993; du Manoir, 1995b). All of the green:red ratio profiles collected across all chromosomes represent the copy number karyotype (ratio comparisons) for test (green) and reference (red) samples. The copy number karyotypes from multiple metaphases are combined to reduce noise from spread variation and enhance "visibility" of true copy number aberrations in a given test sample (Kallioniemi et al., 1994; Piper et al., 1995).

Regions of repeat must be suppressed to accurately measure ratios in adjacent chromosomal regions. Typically, these centromeric, heterochromatic and telomeric regions are partly suppressed with human Cot-1 DNA and salmon testes DNA, but still vary widely in copy number (either extremely high or low) (Kallioniemi et al., 1994; du Manoir et al., 1995b; Ried et al., 1997). This variation in heterochromatin is elevated if the test and reference are of different donors (du Manoir et al., 1993; Kallioniemi et al., 1994). Similar difficulties are encountered with telomeric DNA.

Thompson and Gray suggested that CGH be enhanced by follow-up with FISH to detect or confirm changes in chromosome number, translocations, inversions, or derivative chromosomes (Thompson and Gray, 1993). While this remains true, a more sensitive and revealing analysis on a per-cell basis was made available with the introduction of SKY. In consideration of all of the benefits of CGH, the most notable 
downside to the technique is the inability to detect the chromosomal structure underlying the gains and losses (Houldsworth and Chaganti, 1994). The development of SKY fostered the detection of most structural rearrangements, and allowed for a more complete molecular cytogenetic analysis of tumors when used in conjunction with $\mathrm{CGH}$.

\section{SPECTRAL KARYOTYPING.}

FISH has become an integral technique in cancer research and other cytogenetic diagnoses, but the field of molecular cytogenetics benefits greatly from FISH-based techniques that screen the entire genome (Schröck et al., 1996; Macville et al., 1997; Schröck et al., 1997). SKY is one such technique wherein the structural and numerical analysis of all chromosomes is made possible by a combinatorial labeling scheme and advances in image acquisition and analysis (Schröck et al., 1997; Schröck and PadillaNash, 2000). Unlike CGH, SKY is only applicable to fresh material capable of cell division (Schröck et al., 1996; Ried et al., 1997). One of the most attractive features of this technique, is the ability to identify chromosomal aberrations rapidly and without any previous indication of the abnormalities, as the technique explores the entire genome in a single hybridization (Cohen et al., 1997; Rothmann et al., 1998). In addition, the chromosomal composition of previously unclassified marker chromosomes is revealed (Rothmann et al., 1998; Bayani and Squire, 2001). In essence, SKY is the ideal hybrid of the functionality of CGH and FISH; it provides a screen of an entire genome, like CGH, with the sensitivity of FISH for detection of structural and numerical chromosomal aberrations (Chang and Mark, 1997; Schröck et al., 1997).

In particular, this technique facilitates a differential display of all human chromosomes in a color karyotype using 24 distinct colors, with a resolution of $1-2 \mathrm{Mb}$ 
(Macville et al., 1997; Rothmann et al., 1998; Tonon et al., 2000; Bayani and Squire, 2001). Whole chromosome probes are generated via DOP-PCR and differentially labeled with various combinations of 5 fluorochromes, thus producing 24 distinctly colored paints (Garini et al., 1996; Schröck et al., 1997; Tonon et al., 2000; Schröck and PadillaNash, 2000). Typically, 3 of the fluorochromes are directly incorporated into the probes via fluorochrome-labeled dNTPs (rhodamine, Texas-Red or Spectrum Orange, FITC or Spectrum Green, and Cy3). The other 2 are incorporated via hapten-coinjugated dNTPs, typically detected with avidin Cy5 and mouse antidig Cy5.5, and sometimes amplified further by antibody layering (Garini et al., 1996; Macville et al., 1997; Tonon et al., 2000). Inclusion of unlabeled competitor DNA (i.e. Cot-1 and salmon testes DNA), as previously described for $\mathrm{CGH}$ and FISH, allows for suppression hybridization and contributes to accurate chromosome painting and resolution of chromosome abnormalities (Macville et al., 1997).

As stated earlier, a specific color is assigned to each chromosome using a combination of these direct and indirect labels. SKY is similar to M-FISH, in that each technique uses combinatorial labeling to identify chromosomes. The use of combinatorial labeling allows for the number of targets to exceed the number of fluorochromes, particularly given the breadth of hapten-labeling and detection schemes (Garini et al., 1996; Macville et al., 1997; Ried et al., 1997; Garini et al., 1999). Basic FISH applications are hampered by the fact that the spectra of the available fluorochromes overlap, and therefore it is not possible to define 24 separate probes (Ried et al., 1997). However, M-FISH overcomes this by detecting each fluorochrome separately using a set of applicable filters, and requires separate image captures and 
subsequent overlay prior to image analysis (Speicher et al., 1996). On the other hand, SKY simultaneously excites all fluorochromes and determines the emission spectra, or peak intensity and wavelength, at each pixel in a single image for the entire metaphase spread (Schröck et al., 1996; Ried et al., 1997; Rothmann et al., 1998; Lee et al., 2001).

Spectral imaging, the foundation for SKY, is based upon a combination of spectroscopy and imaging (Garini et al., 1996; Macville et al., 1997; Ried et al., 1997; Rothmann et al., 1998). Spectroscopic analysis is centered around a stained histological specimen that when illuminated, either absorbs or reflects the light, and emits photons revealing the properties of the dye (Rothmann et al., 1998). The hybridization itself is fairly similar to other FISH-based protocols, and only a single CCD image capture is required for SKY analysis. After exposure through a triple bandpass filter, which excites all dyes simultaneously and blocks excitation light from the emission path, a Sagnac interferometer creates an optical path difference in the light emitted from the painted chromosomes (Fig. 9). An interferogram is generated for every pixel during the CCD image capture; this is a measure of emitted spectra in terms of intensity at a given wavelength (Schröck et al., 1996; Garini et al., 1996; Macville et al., 1997; Ried et al., 1997; Raap, 1998; Rothmann et al., 1998; Garini et al., 1999). The differences in the captured spectra may be visualized in the display colors of the CCD image. A Fourier transformation ensures that subtle changes in intensity and wavelength between similar spectra are assigned drastically different classification colors, or pseudo-colors, to allow appropriate discrimination (Schröck et al., 1996; Garini et al., 1996; Macville et al., 1997; Ried et al., 1997; Raap, 1998; Garini et al., 1999; Bayani and Squire, 2001). 
Multiple FISH probes can be used to query several genes at once, but the ability to simultaneously paint all 24 human chromosomes is a powerful tool for performing a global analysis of the chromosome complement of a given cell. Independently, FISH can not accommodate enough spectrally distinct fluorochromes for a global analysis, and SKY will not distinguish submicroscopic deletions, para- or peri-centric inversions, or small duplications or deletions that do not alter the size or composition of the chromosome (Garini et al., 1996; Veldman et al., 1997; Chang and Mark, 1997). Therefore, the combination of SKY and FISH in a single preparation is a very useful tool for looking at the involvement of a specific gene in a rearrangement event, and is a unique refinement on employing each technique individually and attempting to synthesize the results (Tonon et al., 2000). Combination of these 2 techniques allows the researcher to assay the involvement of a specific gene and of specific chromosomes in a given rearrangement using a single preparation. SKY may also be employed as a screening technique for gene or chromosome involvement, perhaps best applied following an expression study such as serial analysis of gene expression (SAGE) or microarray analysis (Ried et al., 1997; Tonon et al., 2000).

Tumors, in particular solid tumors, often have complex karyotypes with multiple rearrangements in the genome (Ried et al., 1997; Tonon et al., 2000). While SKY remains very useful for such applications, it has the potential for misclassification, commonly due to fluorescence signal flaring that obscures a breakpoint or otherwise interferes with interpretation by the SKY software (Lee et al., 2001). Although the resolution of SKY is estimated at $1-2 \mathrm{Mb}$, actual resolution hinges upon the degree of chromatin condensation (Macville et al., 1997; Schröck et al., 1997; Bayani and Squire, 


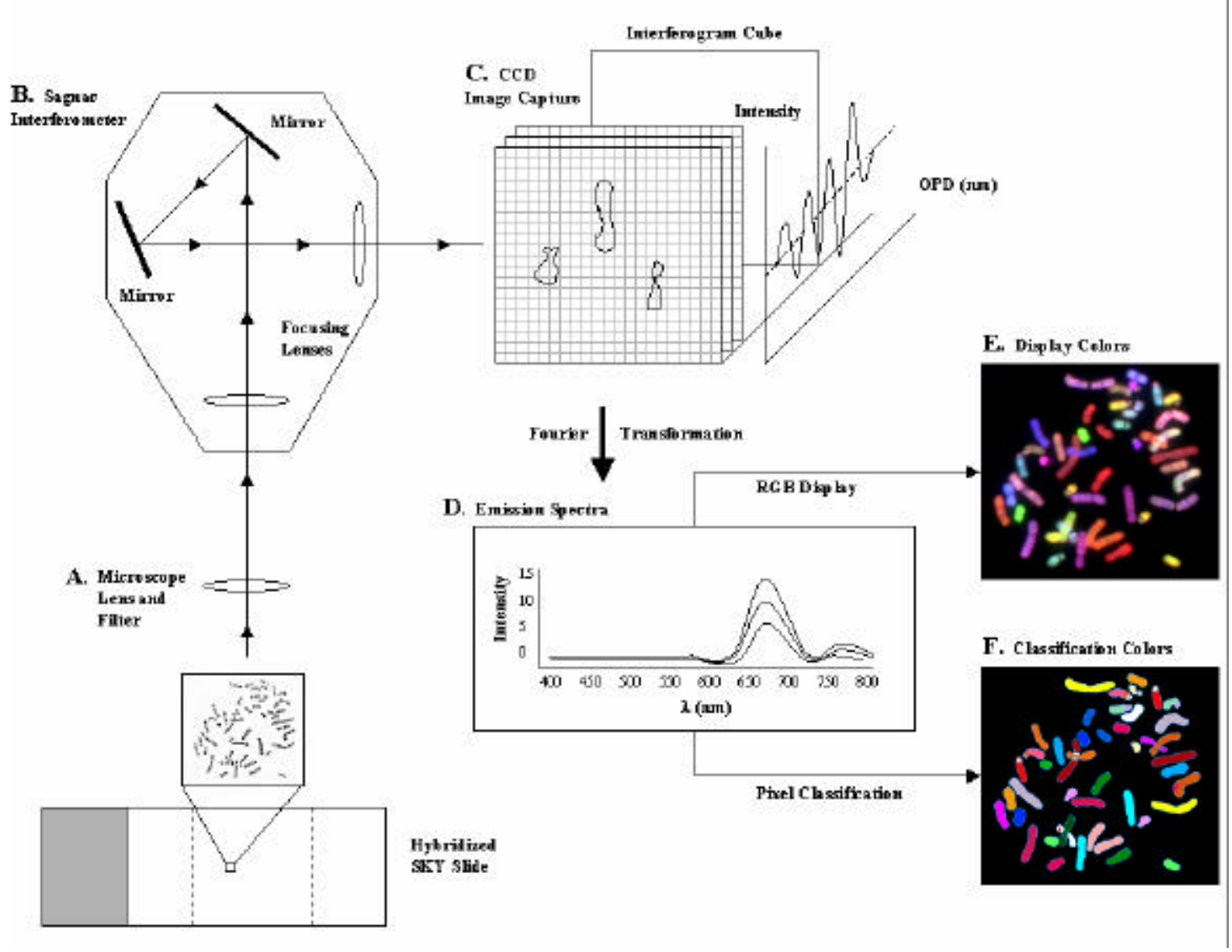

Figure 9. Schematic for SKY Image Capture and Analysis. The principle of spectral karyotyping is spectral imaging, which allows distinction of spectrally-overlapping fluorochromes in a SKY hybridization. Emitted light is visualized through a triple bandpass filter (A), the light is then passes through a Sagnac interferometer (B), and the image is captured in a single exposure using a CCD camera (C). The interferogram seen in $\mathbf{C}$ ) is the pixelby-pixel detail of wavelength and intensity across the entire captured image. (D) The interferogram is analyzed using Fourier transformation to determine the spectrum of the light at each pixel. The spectra for the entire image can be visualized directly as an RGB image (E) similar to the image seen through the microscope. Alternatively, each pixel is classified and the image is pseudocolored (F) to distinguish overlapping fluorochromes.

This image was adapted from Schrock et al., 1996. 
2001). An additional drawback is the cost associated with SKY hybridization, making it potentially most effective as an initial screen of a number of cells prior to a thorough analysis with a specific target for FISH or a comprehensive CGH analysis (Lee et al., 2001; Bayani and Squire, 2001). The combination of SKY with CGH allows for an amazingly powerful analysis to resolve structural and numerical chromosome aberrations, at which point the inclusion of FISH becomes useful as a confirmatory assay (Macville et al., 1999).

\section{UveAL MeLANOMA.}

Uveal melanoma is the primary intraocular tumor observed in adults, and often results in blindness and/or death. Epidemiological studies show an incidence of approximately 6 per million per year in the Caucasian population; within this population it is more common in lightly pigmented individuals (McLean and Gamel, 1996). Lightly pigmented eyes are also demonstrated to be more susceptible to the melanoma (Regan et al., 1999). The clinical course of the cancer is related to the size of the tumor, the cell type, and the variability of the nucleolar size (Gordon et al., 1994; Prescher et al., 1996). Additional considerations include gender, wherein males are slightly more susceptible than females, and predisposing factors (McLean and Gamel, 1996).

Previous studies have shown that oculo(dermal) melanocytosis, neurofibromatosis type 1, Li-Fraumeni syndrome, and familial atypical mole and melanoma (FAM-M) syndrome are predisposing factors (Singh et al., 1996a; Kincaid, 1998). Due to the strong link between sunlight and cutaneous melanoma, researchers have also considered sunlight as a potential cause for uveal melanoma; the results have been ambiguous (Tucker et al., 1985; Egan et al., 1988; Holly et al., 1990; Dolin et al., 1994). There have 
been rare cases of familial uveal melanoma, thought to be inherited in an autosomal dominant fashion and as yet unlinked to any genetic locus or cytogenetic anomaly (Lynch et al., 1968; Singh et al., 1996a; Singh et al., 1996b).

In relation of gross pathology to prognosis, the most significant feature of this solid tumor is the largest tumor dimension (LTD) (Hungerford, 1993; McLean and Gamel 1996). Lesions are considered small if the LTD is at or below 10mm, medium if the LTD is $11-15 \mathrm{~mm}$, and large if the LTD exceeds $15 \mathrm{~mm}$ (McLean and Gamel, 1996). The prognosis worsens as the tumor increases in size. Other features, including vascular supply, are far less significant (Kincaid, 1998).

Uveal melanomas may arise in the choroid, ciliary body, or the iris (Fig. 3). The origin of the tumor has prognostic significance, with melanomas originating in the iris having the most favorable prognosis (Hungerford, 1993; Kincaid, 1998). Iris melanomas are the least common, while choroidal melanomas are the most common (Hungerford, 1993; McLean and Gamel, 1996; Kincaid, 1998; Singh et al., 2001). Choroidal melanomas often rupture the Bruch's membrane and invade the subretinal space, giving them a characteristic mushroom shape (McLean and Gamel, 1996; Kincaid, 1998). Ciliary body tumors can later invade the iris. Clinical diagnosis may occur following routine examination of a asymptomatic eye, blurring or loss of vision, pain from inflammation, or retinal detachment and intraocular hemorrhage (McLean and Gamel, 1996).

Uveal melanoma tumors are generally classified based on cell type, using a modified version of the Callender classification system (Callender, 1931; McLean et al., 1983; McLean and Gamel, 1996). In this system, uveal melanomas containing 
epithelioid cells are designated "mixed-cell type," melanomas without epithelioid cells are termed "spindle-cell type," and tumors with too much necrosis to be classified by cytology are designated "necrotic type."

Approximately $50 \%$ of patients die from hematogenous metastasis (McLean and Gamel, 1996). Metastasis occurs hematogenously, due to the lack of a lymph system in the eye, and almost exclusively localizes to the liver. The preferential metastasis to the liver is not well understood, but can not be explained through lymphatic drainage pathways (McLean, 1995).

The process of hematogenous metastasis is sequential, where each step is fully completed before the following step can take place. Interruption of the metastatic process translates into a cure for the patient. The stepwise process of hematogenous metastasis is as follows: growth and local invasion of primary site, neovascularization, vasular invasion, embolization, transport to the metastatic site, arrest at the metastatic site, extravascularization at the metastatic site, growth and neovascularization, growth of tumor cells in newly established metastasis, and finally re-metastasis (Fidler, 1990; McLean, 1993). Survival time is primarily a function of the growth rate of tumor cells, because this determines the pace of the metastatic process. The significance of lead time and tumor burden (also known as LTD) are thereby decreased in uveal melanoma. Lead time is the length of time between metastasis and treatment, and its significance is decreased because metastases are often not discovered prior to removal of the eye. The relevance of tumor burden on survival time is decreased, because the exponential growth of a tumor has a larger effect on tumor size than pronosis since the critical number of doubling times before metastasis can ot be estimated (McLean, 1993). In addition, 
patient to patient variation in tumor size decreases the strength of this prognostic indicator. The mean of the diameters of the 10 largest nucleoli, measured from at least 50 fields of the tumor, has been found to be a good predictor of survival; "the larger the nucleoli the lower the cure-rate and the shorter the median survival time" (McLean, 1993; McLean, 1995; Periera et al., 2001). This measurement is similar, but slightly different, in cutaneous melanoma, where the mean of the 10 largest nuclei, rather than nucleoli, is of prognostic significance (Periera et al., 2001). Survival time following metastasis is also dependent on the immunologic defense system of the host, which is primarily tumorinfiltrating lymphocytes (TIL). In most solid tumors, presence of TIL are associated with a better prognosis; for indeterminate reasons, the opposite is true when infiltration occurs in uveal melanoma (McLean, 1993; McLean, 1995; McLean and Gamel, 1996).

The primary treatment for uveal melanoma is enucleation, or removal of the eye. Conservative therapies include radiation and eye-wall resection, with the intent of preserving the eye and maintaining vision (McLean and Gamel, 1996; Kincaid, 1998). Radiation therapy is done using a precisely focused particle beam or a radioactive plaque sutured to the sclera beneath the tumor. Eye-wall resection can be used on medium or small tumors $(\leq 15 \mathrm{~mm})$, but is not feasible on large tumors $(>15 \mathrm{~mm}$ ) (McLean and Gamel, 1996). Survival studies are in progress to compare prognosis and survival following enucleation or more conservative therapies, such as radiation and resection (Hungerford, 1993; McLean and Gamel, 1996). The conservative treatments are currently favored in smaller tumors, which researchers hope will one day result in tumor control and preservation of vision (Kincaid, 1998). 
Investigations at the sub-cellular level have begun to unveil the genetics underlying tumor development, progression, and metastasis. Cytogenetic evidence indicates that copy number abnormalities prevail within uveal melanoma (Gordon et al., 1994; Speicher et al., 1994; Ghazvini et al., 1996). Results from cytogenetic analyses suggest that the most frequent abnormalities occur in chromosomes 3, 6, and 8; monosomy 3 is associated with a poor prognosis (Speicher et al., 1994; Prescher et al., 1996; White et al., 1998a). Monosomy 3 is seen in uveal melanoma of the ciliary body, whereas disomy or polysomy of chromosome 3 are reported in choroidal melanoma (Sisley et al., 1993; McNamara et al., 1997). Abnormalities of chromosome 6 are also seen in cutaneous melanoma, which suggests the cancers may share some common genetic alterations (Griffin et al., 1988). Other recurrent abnormalities have been noted with varying consistency, including those on 7q, 9p, and 13q (Gordon et al., 1994).

Evidence suggests a bifurcated pathway for tumor progression, wherein an aberration of either chromosome 3 or 6 occurs, eventually followed by alteration of chromosome 8 and metastasis. Chromosome 8 abnormalites are not seen unless accompanied by an aberration of chromosome 3 or 6 (Parella et al., 1999).

Due to its strong link with prognosis, chromosome 3 copy number is frequently assayed in the uveal melanoma research community. Monosomy 3 was first identified using classical cytogenetic techniques, and was generally reported in 50\% of the tumors studied (Horsman et al., 1990; Sisley et al., 1990; Prescher et al., 1990; Horsthemke et al., 1992; Sisley et al., 1992; Horsman and White, 1993; Wiltsire et al., 1993; Singh et al., 1994; Prescher et al., 1994; Prescher et al., 1995; Prescher et al., 1996; White et al., 1998b; Parada et al., 1999; Sisley et al., 2000; Tschentscher et al., 2001). Similar results 
were found by hybridization-based techniques, including analyses of chromosome 3 loss using FISH (Wiltshire et al., 1993; Sisley et al., 1997; Naus et al., 2002). CGH studies report monosomy with similar frequency as well (Speicher et al., 1994; Gordon et al., 1994; Prescher et al., 1996; Ghazvini et al., 1996; Tschentscher et al., 2001; Naus et al., 2001). Studies employing a combination of these methods offer an internal validation of their findings (Wiltshire et al., 1993; Prescher et al., 1996; Tschentscher et al., 2001). However, the comprehensive cytogenetic characterization of uveal melanoma remains incomplete, largely because most studies involve small sample sizes almost exclusively comprised of fresh material.

The absence of chromosome 3, 6, and 8 abnormalities in some cases, suggests that there are additional pathways of malignant transformation (Singh et al., 1996a). Several papers seem to refute or question the bifurcated model of development (White et al., 1998b; Sisley et al., 2000; Naus et al., 2001). This indicates that while there may be exclusivity with regard to the sequence in which chromosome abnormalities appear in some tumors, it does not hold true in every case. Alternatives to monosomy 3 have also been reported recently, and are starting to receive some attention. Decreases in copy number of chromosome 3 have sometimes been reported in lieu of monosomy, where CGH showed a tendency toward loss of a portion of the chromosome rather than complete loss (Gordon et al. 1994; Ghazvini et al., 1996). LOH studies have demonstrated losses on either arm of the chromosome rather than along the length of the entire chromosome (Sisley et al., 1993; White et al., 1998a; Parella et al., 1999; Myatt et al., 2000; Tschentscher et al., 2001). Furthermore, isodisomy of chromosome 3, trisomy 
3, and tetrasomy 3 have been reported in the literature (McNamara et al., 1997; White et al., 1998a). 


\section{MATERIALS and METHODS}

\section{Human Peripheral BLOOD L YMPHOCYTe (HPBL) CULTURE.}

Venipuncture was performed on healthy and karyotypically normal male and female donors with informed consent, and $0.8 \mathrm{ml}$ blood was added to $10 \mathrm{ml}$ RPMI 1640 media (Invitrogen Life Technologies, Carlsbad, CA) supplemented with 20\% FBS (Sigma Co., St. Louis, MO), $100 \mu 1$ penicillin/streptomycin $(100 \mathrm{U} / \mathrm{ml}$ and $100 \mu \mathrm{g} / \mathrm{ml}$, respectively) and 1\% sodium heparin (Invitrogen Life Technologies, Carlsbad, CA). Then, $216 \mu 1$ phytohemagglutinin (PHA) (Invitrogen Life Technologies, Carlsbad, CA) was added to each T-25 culture. The cultures were incubated at $37^{\circ} \mathrm{C}$ in $5 \% \mathrm{CO}_{2}$ for 72 hours.

\section{UVEal Melanoma CeLl Lines.}

Ten uveal melanoma cell lines were obtained from the Department of Ophthalmology, University of Texas Southwestern Medical Center at Dallas (Dallas, TX), and maintained in culture using standard techniques (Table 1). The cell lines were derived from 8 primary uveal melanomas and 2 metastases.

\section{Metaphase Chromosome Preparations.}

We prepared metaphase chromosome spreads from normal, cultured human peripheral blood lymphocytes (HPBL) and cultured cell lines via conventional techniques (Tucker et al., 1994). After 72 hours of culture, $0.1 \mu \mathrm{g} / \mathrm{ml}$, or $100 \mu 1$, of colcemid (Karyomax Colcemid Solution, Invitrogen Life Technologies, Carlsbad, CA) was added to each culture flask. Cultures with colcemid were incubated an additional 15 minutes, after which the T-25 cultures were gently agitated and transferred to $15 \mathrm{ml}$ conical centrifuge tubes, and centrifuged at 1200rpm for 8 minutes. The supernatant was 
aspirated, the cell pellet resuspended in $5 \mathrm{ml}$ of a $75 \mathrm{mM} \mathrm{KCl}$ (see appendix) hypotonic solution pre-warmed to $37^{\circ} \mathrm{C}$, and incubated for 20 minutes at $37^{\circ} \mathrm{C}$. The hypotonic solution was used to swell the cells, eventually causing the cells to burst and release their chromosomes when dropped onto slides, forming metaphase spreads. Following incubation, the addition of $2 \mathrm{ml}$ fresh Carnoy's fixative (see appendix), and subsequent centrifugation for 8 minutes at 1200rpm, stopped the swelling of the cells. The supernatant was again aspirated, and the cells resuspended in 5-10ml fresh fixative. The cells were centrifuged, the supernatant aspirated, and the pellet resuspended. This process was repeated until the cell pellet appeared to be clean and completely white upon visual inspection.

At this point the cells were dropped onto slides; alternatively, the cells were resuspended in fixative, stored upright at $4^{\circ} \mathrm{C}$ and dropped onto slides within the next few days. The best results were observed when slides were dropped on the same day as the harvest. In order to make metaphase slide preparations, the supernatant was aspirated and the cells resuspended in approximately $0.5-1 \mathrm{ml}$ glacial acetic acid with a $200 \mu 1$ pipette after the final centrifugation. A clean slide, swabbed with ethanol until free of debris, was placed over an $80^{\circ} \mathrm{C}$ water bath and misted with $\mathrm{dH}_{2} \mathrm{O}$. The pipette tip was loaded, and 2 adjacent drops of the cell suspension were placed onto the slide from a height ranging from 6 inches to 3 feet above the slide. The slide was dried over the water bath, checked for quality, stored in a slide box to age for approximately 1 week. For long-term storage, aged slides were kept in a slide box with desiccant, flushed with gaseous nitrogen, sealed in a plastic bag and kept at $-20^{\circ} \mathrm{C}$ until needed. 


\section{NuClei PREPARATions.}

For FISH analysis, nuclei from the uveal melanoma cell lines and a normal male donor were harvested from $25 \mathrm{ml}$ culture flasks using the procedure for metaphase spread preparation, but in the absence of colcemid (Tucker et al., 1994). Nuclei preparations were aged and stored in the same manner as the metaphase preparations.

\section{UVEal Melanoma Tumor CASES.}

One hundred FFPE uveal melanoma tumors were selected at random from the AFIP tissue archives. Two $6 \mu \mathrm{m}$ tissue sections per case were available for microdissection, and were accompanied by photocopies of corresponding $\mathrm{H} \& \mathrm{E}$ stained slides for tumor identification (Fig. 4). Of these cases, 51 patients (23 male, 28 female) survived more than 9 years without evident metastasis, and the remaining 49 patients (29 male, 20 female) succumbed to metastatic disease. Determination of gender was required for appropriate hybridization, but no other patient characteristics were revealed until completion of the analysis.

\section{MICRODISSECTION.}

Initial approaches involved using a sterile razor blade to scrape the entire paraffin tissue section from the slide for DNA isolation. It was found that the quality of the DNA was improved if the tumor was exclusively excised from the slide. Microdissection is a useful technique for ensuring the highest possible proportion of tumor cells, with as few contaminating normal cells in the sample as possible (du Manoir et al., 1993; Kallioniemi et al., 1994). Microdissection was performed on two $6 \mu \mathrm{m}$ tissue sections for each of 100 uveal melanoma cases. The accompanying $\mathrm{H} \& \mathrm{E}$ slides were used to locate and isolate the uveal melanoma tumor in the FFPE tissue section. A sterile 20-gauge needle, a sterile 
razor blade, or a fresh microtome blade was used in manual microdissection of the tumor tissue from the slide. The tumor tissue from both tissue sections was combined in a single $1.7 \mathrm{ml}$ microcentrifuge tube for each case to ensure the availability of sufficient tumor DNA for DOP-PCR; this was a concern due to the variability in tumor size from case to case.

\section{DNA ISOLATION.}

\section{Normal (reference) DNA.}

Reference samples for the entire study were collected via venipuncture from a single normal male and a single normal female donor with informed consent, and the majority of blood was aliquoted into T-25 flasks for culture of HPBL as previously described. A small volume of blood was set aside for isolation of whole genomic DNA; the addition of $200 \mu 1$ whole blood to a microcentrifuge tube yielded approximately $35 \mu 1$ purified DNA upon completion of the isolation protocol.

The isolation protocol began with the addition of $20 \mu 1$ RNase A (see appendix) (Roche Molecular Biochemicals, Indianapolis, IN) to $200 \mu 1$ whole blood, mixed by vortexing. Then, $25 \mu 120 \mathrm{mg} / \mathrm{ml}$ Proteinase K (Roche Molecular Biochemicals, Indianapolis, IN) and $200 \mu 1$ cell lysis buffer (see appendix) were added to the tube, vortexed thoroughly, and incubated for 10 minutes at $70^{\circ} \mathrm{C}$. Proteinase $\mathrm{K}$ digestion increased the yield of high molecular weight DNA (Isola et al., 1994). Then, 500ml phenol/chloroform/isoamyl alcohol solution (PCI) (25:24:1, v/v) (Invitrogen Life Technologies, Carlsbad, CA) was added to the tube(s) after removal from the oven, and then the tube was vortexed again. This was followed by a full speed (14,000rpm) centrifugation for 5 minutes in a microcentrifuge. The upper aqueous layer was removed 
and put into a clean microcentrifuge tube, and another $500 \mu 1$ PCI was added, again followed by vigorous vortexing. The tube(s) was spun at full speed for another 5 minutes. The upper layer was again removed and placed into a clean tube.

The following reagents were then added to this solution: $25 \mu 13 \mathrm{M}$ sodium acetate (Quality Biological, Inc., Gaithersburg, MD), 1.5 $\mu 1$ 20mg/ml glycogen (Roche Molecular Biochemicals, Indianapolis, IN), and $100 \%$ ethanol at volume equal to $3 \mathrm{x}$ the volume of the tube. The tube was again vortexed to ensure an evenly distributed solution, and incubated at $-20^{\circ} \mathrm{C}$ for up to 2 hours. The DNA was precipitated via full speed centrifugation for 5 minutes. The supernatant was aspirated, $0.5 \mathrm{ml} 85 \%$ ethanol added to the pellet, and vortexed thoroughly. Following a second 5 minute centrifugation at full speed, the ethanol was decanted, and the $85 \%$ ethanol wash repeated. The resulting supernatant was aspirated, and the pellet of DNA in the bottom of the tube was dried in a lyophilizer set on high for 5 minutes. The pellet was finally resuspended in $35 \mu 1 \mathrm{TE}$ buffer ( $\mathrm{pH}$ 7) (see appendix) for long-term storage at $-20^{\circ} \mathrm{C}$. DNA quantification was performed using a GeneQuant II (Pharmacia Biotech, Cambridge, England); the 260/280 ratios and DNA concentration recorded.

\section{Cell Line DNA.}

DNA was extracted from uveal melanoma cell lines maintained in culture. The media was removed from the T-25 flask and set aside. Two milliliters of 1xTrypsinEDTA (Mediatech, Inc., Herndon, VA) were added to the flask, and when the cells were fully suspended in the Trypsin-EDTA solution, the media was replaced into the flask. The contents of the flask were pipetted into a $15 \mathrm{ml}$ conical centrifuge tube, and centrifuged at $1500 \mathrm{rpm}$ for 6 minutes. The supernatant was aspirated, and the cell pellet 
was washed twice in $10 \mathrm{ml} 1 \mathrm{xPBS}$ (w/o $\mathrm{MgCl}$ or $\mathrm{CaCh}_{2}$ ); each time the supernatant was aspirated. The cell pellet was resuspended in approximately $1 \mathrm{ml}$ of $1 \mathrm{xPBS}$ (w/o $\mathrm{MgCh}$ or $\mathrm{CaCl}_{2}$ ) following the second wash. The sample was pipetted from the $15 \mathrm{ml}$ conical tube into microcentrifuge fuge tubes in $200 \mu 1$ aliquots. From this point on, the DNA isolation procedure was identical to that described for normal HPBL DNA.

\section{Tumor (test) DNA.}

Following microdissection, as previously described, tumor DNA was extracted via a protocol similar to that described earlier for normal DNA. First, 800 $\mu 1$ Hemo-De (Fisher Scientific, Inc., Pittsburgh, PA) was added to each tube containing microdissected tumor tissue. Following addition of Hemo-De, the tubes were vortexed, and $400 \mu \mathrm{l}$ ethanol were added. The tube was vortexed again, and centrifuged at full speed for 5 minutes. The supernatant was decanted, and $800 \mu l$ ethanol was added. Once again, the tube was vortexed and centrifuged at full speed for 5 minutes. The supernatant was decanted, and the pellet dried in a $55^{\circ} \mathrm{C}$ oven for 5 minutes. Then, $250 \mu 1$ cell lysis buffer and $25 \mu 120 \mathrm{mg} / \mathrm{ml}$ Proteinase $\mathrm{K}$ were added to the tube, and vortexed thoroughly. The tube(s) was incubated overnight at $55^{\circ} \mathrm{C}$ in an Eppendorf Thermo Mixer (Brinkmann Instruments, Inc., Westbury, NY). The remainder of the DNA isolation protocol, from PCI extraction through DNA quantification, was identical to that described for normal DNA.

\section{NiCK TRANSLATION.}

For the cell line CGH experiments, all whole genomic probes were generated via nick translation of extracted DNA (Fig. 10). Each $50 \mu$ l nick translation reaction contained the following reagents: $1 \mu \mathrm{g}$ DNA (volume variable), $5 \mu 110 x N T$ buffer (see 


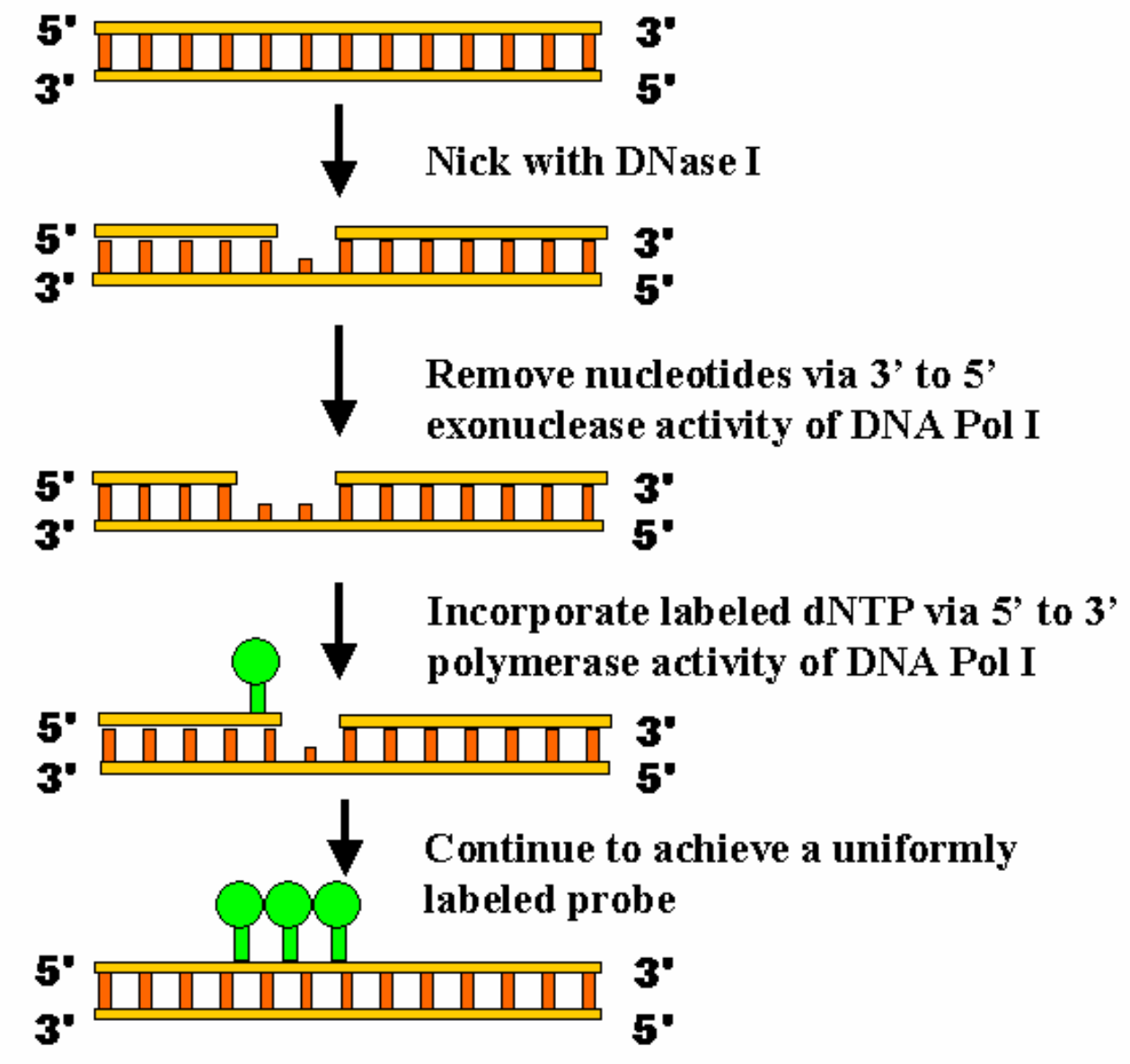

Figure 10. Probe Production via Nick Translation. Genomic DNA is randomly nicked with Dnase I, after which DNA Polymerase I excises nucleotides in the 3' to 5' direction. Then, the same polymerase will replace the patch of excised nucleotides using the available pool of dNTPs. Due to the decreased ratio of dTTP in the dNTP mixture, hapten- or fluorochroms -labeled dUTP will be preferentially incorporated. The dUTP is labeled, but the preferential incorporation is not skewed enough to cause steric hindrance of the resulting labeled probe DNA.

This figure courtesy of Dr. Alison E. Director-Myska, AFIP. 
appendix), $5 \mu 1 \mathrm{dNTP}$ mixture (see appendix), $5 \mu 1$ 0.1M B-Mercaptoethanol (see appendix), $2 \mu 1$ of either biotin-16-dUTP or digoxigenin-11-dUTP (Roche Molecular Biochemicals, Indianapolis, IN), with the remainder being sterile $\mathrm{H}_{2} \mathrm{O}$. The solution was vortexed, $5 \mu 1$ 1:1000 DNase I (see appendix) (Roche Molecular Biochemicals, Indianapolis, IN) and $1 \mu 1$ Kornberg polymerase (Roche Molecular Biochemicals, Indianapolis, IN) were added. The reaction tubes were incubated for 2 hours at $15^{\circ} \mathrm{C}$. Following the incubation, $5 \mu 1$ samples of each reaction were run on a $1.7 \%$ agarose gel (see appendix) to size the fragments. After the labeled DNA fragments were sized by agarose gel electrophoresis and ethidium bromide visualization by UV (ultraviolet) excitation, optimal probe size was seen as a smear of DNA between 200- and 1000-bp in length (Fig. 11) (Kallioniemi et al., 1994; Houldsworth and Chaganti, 1994). To load the gel, $5 \mu 1$ nick translation product were added to $0.5 \mu 1$ loading buffer (see appendix), and at least 1 well was loaded with a 100bp ladder (Roche Molecular Biochemicals, Indianapolis, IN). If the probe length exceeded 1000bp in length, additional DNase I was added, and the reaction continued at $15^{\circ} \mathrm{C}$ with size checks every 30 to 60 minutes. If the probe was smaller than $200 \mathrm{bp}$, it was discarded and a new reaction was set-up. Once the probe was in the appropriate size range, $1 \mu 10.5 \mathrm{M}$ EDTA (Digene, Gaithersburg, MD) was added and tubes were incubated at $65^{\circ} \mathrm{C}$ for 10 minutes to halt the reaction; the probes were available for immediate use, or could be stored at $-20^{\circ} \mathrm{C}$ until needed.

\section{Polymerase Chain ReaCtion (PCR).}

Whole genomic probes, for the tumor study, were generated through DOP-PCR, as first described by Telenius and colleagues (Fig. 8) (Telenius et al., 1992a and b). DOP-PCR, accepted as an accurate method of probe creation for CGH, results in larger 


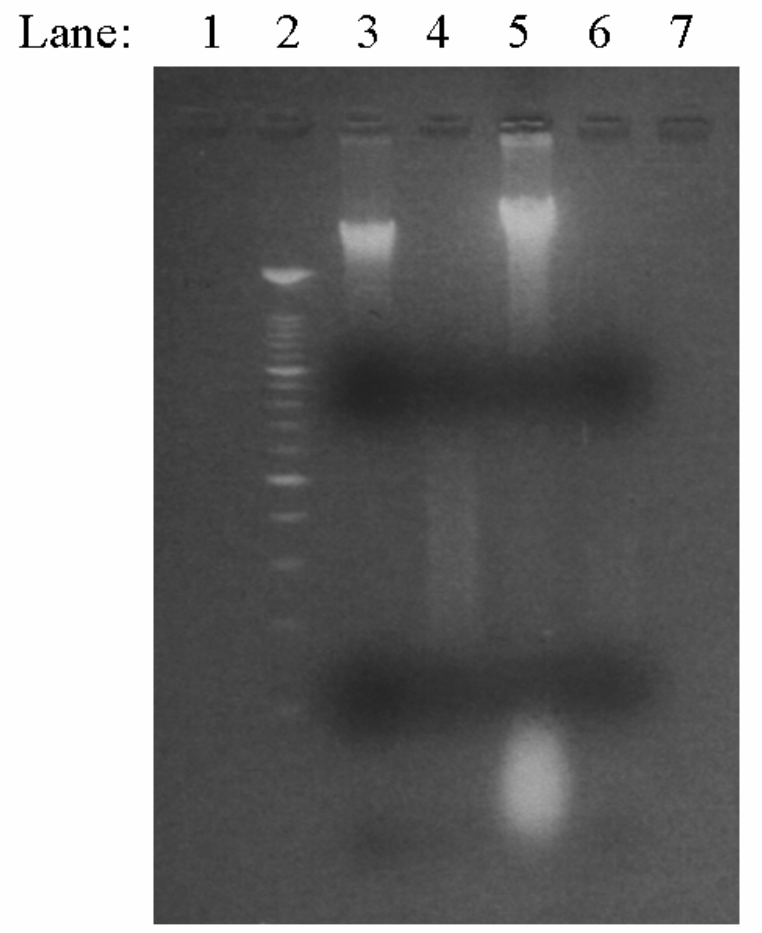

Figure 11. Agarose Gel Electrophoresis Comparing DNA Extracts with Nick Translation Products. Phenol/chloroform/isoamyl alcohol (PCI) extraction procedures were used to isolate normal DNA from normal male and female donors, as well as each of ten uveal melanoma cell lines. Whole genomic probes were generated through nick translation of extracted DNA, and normal DNA and tumor DNA genomic extracts were labeled with digoxigenin-11-dUTP and biotin-16 dUTP, respectively (Roche Molecular Biochemicals, Indianapolis, IN). The resultant labeled DNA fragments were measured via agarose gel electrophoresis, visualized with ethidium bromide staining coupled with visualization through UV excitation, and determined to be between 200 and 1000 base pairs in length. Lane 2 is the DNA molecular weight marker XIV 100 base pair ladder (Roche Molecular Biochemicals, Indianapolis, IN). Lanes 3 and 4 are normal male DNA extract and nick-translated male DNA, respectively. Lanes 5 and 6 are cell line extract from an uveal melanoma cell line and the nick-translated probe product, respectively. 
labeled fragments than nick translation (Kallioniemi et al., 1994). This technique, frequently applied when there was a small volume of the template DNA available, reliably amplifies quantities of DNA as small as 100pg (Speicher et al., 1993). Haptenlabeled dUTP was included in the DOP-PCR reaction mix to allow probes to be visualized indirectly (Fig. 12). Reference and tumor DNA were labeled with digoxigenin-11-dUTP and biotin-16-dUTP, respectively.

DOP-PCR primers were obtained with the sequence 5'- GAT CAA GCT NNN NNN ATG TGG - 3' (Integrated DNA Technologies, Inc., Coralville, IA). Amplification was performed using a GeneAmp ${ }^{\circledR}$ PCR System 9700 thermocycler (PE Applied Biosystems, Foster City, CA). Each $100 \mu$ DOP-PCR reaction mixture contained the following reagents: $10 \mu 1$ of a 1:25 dilution of test or reference DNA in TE Buffer, $10 \mu 1$ 10x PCR Buffer II (w/o MgCh) (Roche Applied Biosystems, Foster City, CA), $10 \mu 1$ 20mM DOP Primer (see appendix), $24 \mu \mathrm{l} 25 \mathrm{mM} \mathrm{MgCb}$ (Roche Applied Biosystems, Foster City, CA), $5 \mu 1$ biotin- or dig-dNTP mixture (see appendix), $39 \mu 1$ sterile $\mathrm{H}_{2} \mathrm{O}$, and $2 \mu 1$ AmpliTaq Gold (see appendix) (Roche Applied Biosystems, Foster City, CA). All reagents, except the DNA, can be combined into a DOP-PCR master mix (PCR MM) (see appendix) to simplify PCR set-up. Conditions were as follows: $95^{\circ} \mathrm{C}$ for 10 minutes initial soak, 15 cycles of $94^{\circ} \mathrm{C}$ for 1 minute, $30^{\circ} \mathrm{C}$ for 1 minute, $72^{\circ} \mathrm{C}$ for 1 minute, and then 60 cycles of $94^{\circ} \mathrm{C}$ for 30 seconds, $52^{\circ} \mathrm{C}$ for 30 seconds, $72^{\circ} \mathrm{C}$ for 1 minute with a 1 second per cycle addition to each step in the 60 cycles, and completed with a final extension of $72^{\circ} \mathrm{C}$ for 10 minutes before a hold at $4^{\circ} \mathrm{C}$. Labeled DNA fragments were sized by agarose gel electrophoresis and ethidium bromide visualization by UV 
excitation. Optimal probe size was seen as a smear of DNA between 200- and 1000-bp in length (Kallioniemi et al., 1994; Houldsworth and Chaganti, 1994).

\section{COMPARATIVE GENOMIC HYBRIDIZATION.}

In brief, slides were first pretreated in order to remove unwanted cytoplasm and protein from the metaphase preps. Indirectly-labeled reference and tumor probes were simultaneously hybridized to the normal metaphase chromosomes. Following a 2-night hybridization, the indirectly-labeled probes were detected using antibody layering detection procedures, and as a final step, the slides were counterstained and coverslipped prior to visualization via fluorescence microscopy (Fig. 13).

Slide pretreatment for hybridization involved several steps. Initially, slides were equilibrated in $2 x \operatorname{xSC}$ (see appendix) at room temperature. Following a short equilibration period, $200 \mu \mathrm{l}$ of a 1:200 dilution of $20 \mathrm{mg} / \mathrm{ml}$ RNase A stock (see appendix) in $2 \mathrm{xSSC}$ was applied to each slide, and the slides were incubated in a hybridization chamber at $37^{\circ} \mathrm{C}$ for 1 hour. Three washes in $2 \mathrm{xSSC}$ for 5 minutes each, complete the digestion of RNA from the slide. Then, slides were treated with a solution prepared from $15 \mu 1100 \mathrm{mg} / \mathrm{ml}$ pepsin stock solution (see append ix) added to $50 \mathrm{ml}$ of pre-warmed $37^{\circ} \mathrm{C}$ $0.01 \mathrm{M} \mathrm{HCl}$, and the slides incubated in a $37^{\circ} \mathrm{C}$ oven for 6 minutes. After the pepsin/ $\mathrm{HCl}$ treatment (see appendix), the slides were washed twice in 1xPBS (w/o $\mathrm{MgCh}$ or $\mathrm{CaCh}$ ) (see appendix) for 5 minutes, followed by a third 5 -minute wash in $1 \times \mathrm{PBS} / \mathrm{MgCh}$ (see appendix). Postfixation was performed in a $50 \mathrm{ml}$ solution of $1 \%$ formaldehyde in $1 \mathrm{xPBS} / \mathrm{MgCh}$ (see appendix) for 10 minutes, followed by a single 5-minute wash in 1xPBS (w/o $\mathrm{MgCl}_{2}$ or $\mathrm{CaCl}_{2}$ ) prior to slide dehydration in a series of 3 ethanol dilutions (70\%, 85\%, and $100 \%)$ for 3 minutes apiece, which completes the slides. 


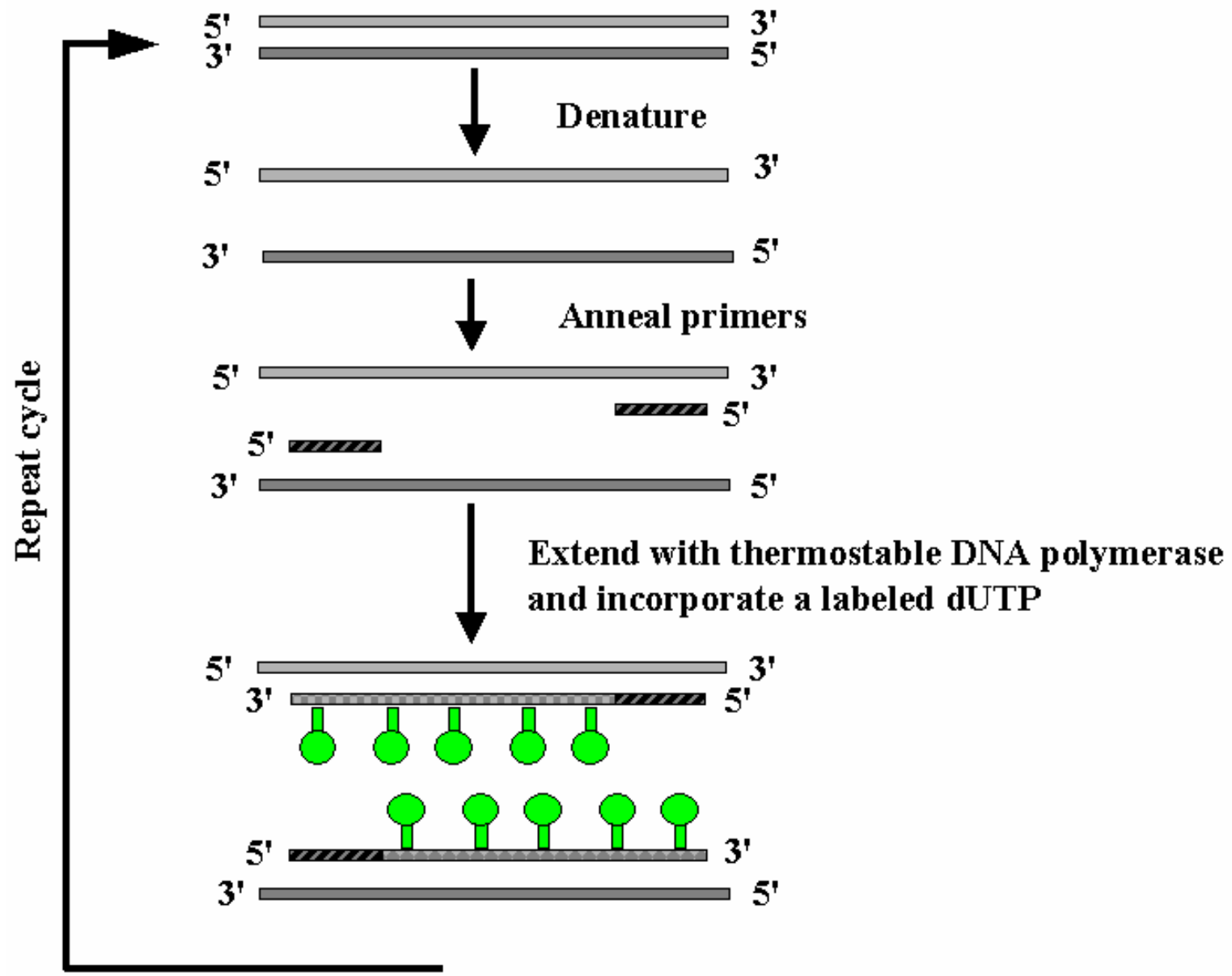

Figure 12. DNA Probe Production via PCR. The process of PCR, simplified, is a series of DNA denaturation, annealing of primers, and extension of the DNA. The primers are specific for sequences within the target DNA to be amplified. When two primers bind within an allowable proximity, the polymerase will create complementary lengths of DNA in the 5' to 3' direction. If a labeled dUTP is incorporated in the dNTP mixture, and the ratio of dTTP is reduced, the dUTP will be incorporated with increased frequency without resulting in steric hindrance. Repetition of the cycle many times amplifies the pool of labeled target DNA probe sequence.

This figure courtesy of Dr. Alison E. Director-Myska, AFIP. 


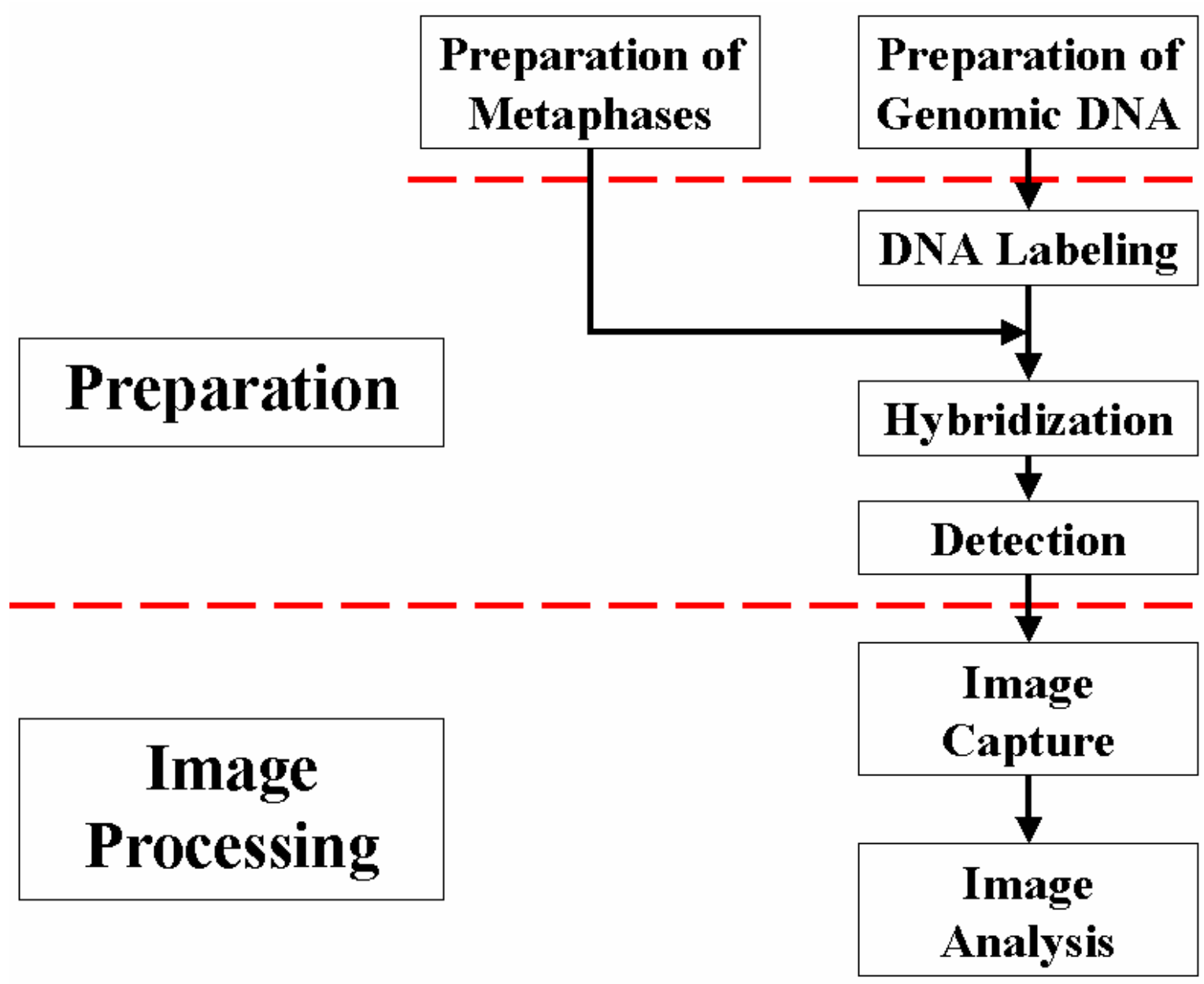

Figure 13. A Flowchart for CGH. This is intended as a general description of the steps for CGH, and is elaborated upon in Figures 14, 15, and 18. The preparation of normal target metaphases occurs in parallel with the preparation and labeling of normal and tumor genomic DNA. Both DNA probes are simultaneously hybridized to the pre-treated target slide, and following a 2night hybridization, the indirectly-labeled probes are detected. For image processing, the target metaphase is captured using DAPI, FITC, and TRITC filters. The combination of images captured using each filter creates a CGH overlay image suitable for analysis.

This figure was adapted from Institut für Pathologie, University Hospital Charité, http://amba.charite.de/cgh/protocol/02/prot02.html. 


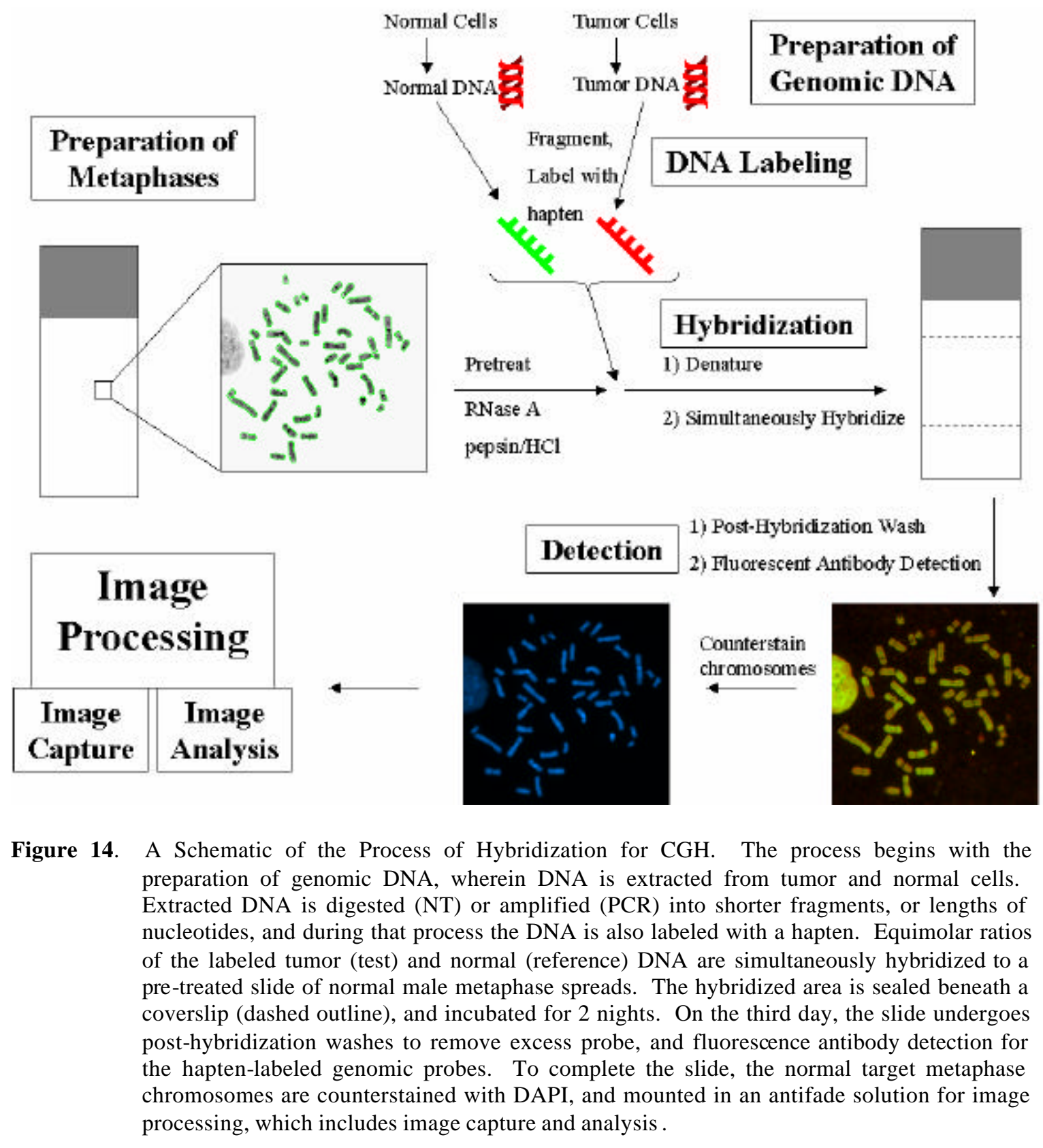


CGH was performed as described by Kallioniemi and colleagues (Fig. 14) (Kallioniemi et al., 1992). The normal and tumor probes, created via DOP-PCR, were combined into a single probe cocktail. To make and precipitate the probe cocktail, $30 \mu \mathrm{l}$ each (tumor and reference) DOP-PCR product, $60 \mu 1$ human Cot-1 DNA (Invitrogen Life Technologies, Carlsbad, CA), $1 \mu 1$ salmon testes DNA (Sigma Co., St. Louis, MO), $12.1 \mu \mathrm{l} 3 \mathrm{M}$ sodium acetate, and $400 \mu \mathrm{l}$ ice cold absolute ethanol were combined in a microcentrifuge tube for each case to be hybridized. The DNA was precipitated by incubation at $-20^{\circ} \mathrm{C}$ for 2 hours. Precipitate was collected by a 30 -minute centrifugation at 14,000rpm. The supernatant was decanted, and the probe pellet was lyophilized for 5 minutes. Then, $5 \mu 1$ molecular grade formamide (Fisher Scientific, Inc., Pittsburgh, PA) was added to each pellet, vortexed, and incubated at $37^{\circ} \mathrm{C}$ for 30 minutes. Afterwards, $10 \mu 1$ hybridization master mix (see appendix) was added, the mixture was vortexed and denatured at $80^{\circ} \mathrm{C}$ for 5 minutes, followed by a 2-hour pre-anneal at $37^{\circ} \mathrm{C}$. One hour and forty minutes into the preanneal, $100 \mu \mathrm{l}$ of $70 \%$ formamide/2xSSC (FA/SSC) (see appendix) was added to each pretreated slide along with a $24 \times 60 \mathrm{~mm}$ glass coverslip. The slides were immediately incubated in an $80^{\circ} \mathrm{C}$ oven for 1.5 minutes, and then dehydrated in a series of ethanol dilutions $(70 \%, 85 \%$, and 100\%) for 3 minutes each. The slides were dried, and the pre-annealed probe applied to the denatured slides. The hybridized slides were then covered with a $22 \mathrm{~mm}^{2}$ coverslip and sealed with Elmer's rubber cement (Elmer's Products, Inc., Columbus, $\mathrm{OH}$ ), and incubated at $37^{\circ} \mathrm{C}$ for 2 nights prior to detection.

On the third day, the solutions for detection were pre-warmed for 1 hour before the procedure was started. Then, the rubber cement and coverslips were removed. The 
slides were then washed at $45^{\circ} \mathrm{C}$ in $50 \% \mathrm{FA} / \mathrm{SSC}$ (see appendix) 3 times for 5 minutes per wash. This was followed by another three 5-minute washes in $0.1 \mathrm{xSSC}$ at $60^{\circ} \mathrm{C}$, and then the slides were dipped in $4 x \mathrm{SSC} / 0.1 \%$ Tween 20 (see appendix) pre-warmed to $37^{\circ} \mathrm{C}$. A blocking solution of $3 \%$ bovine serum albumin (BSA) (Sigma Co., St. Louis, $\mathrm{MO}$ ), pre-warmed to $37^{\circ} \mathrm{C}$, was applied, and the slides were placed in a hybridization chamber at $37^{\circ} \mathrm{C}$ for 1 hour. The slides were again dipped in $4 \mathrm{xSSC} / 0.1 \%$ Tween 20 prewarmed to $45^{\circ} \mathrm{C}$, before the first layer of antibodies was applied. For antibody layering, $100-200 \mu 1$ of a given antibody layer solution was applied to each slide, plastic coverslips were placed over the solution, and the slides were again placed in a hybridization chamber for 1 hour at $37^{\circ} \mathrm{C}$, followed by three 5 -minute washes in $4 \mathrm{xSSC} / 0.1 \%$ Tween 20 pre-warmed to $45^{\circ} \mathrm{C}$. This process of washing, layering, incubating was repeated for 4 layers. The layers were as follows: layer $1=1: 200$ avidin-conjugated FITC (Vector Labs, Burlingame, CA) and 1:100 mouse-anti-DIG (Sigma Co., St. Louis, MO), diluted in 1\% BSA (see appendix); layer $2=1: 200$ biotinylated anti-avidin (Vector Labs, Burlingame, CA) and 1:200 rabbit anti-mouse TRITC (Sigma Co., St. Louis, MO), diluted in 1\% BSA; layer $3=1: 200$ avidin-conjugated FITC in 1\% BSA; and layer $4=$ 1:200 goat anti-rabbit TRITC (Sigma Co., St. Louis, MO). Antibody layering was necessary to amplify the fluorescence signal prior to image capture and analysis. Following antibody detection procedures, slides were counterstained with DAPI (Sigma Co., St. Louis, MO) (see appendix). Finally, a coverslip was mounted over an antifade solution (see appendix) to complete each of the slides.

While some copy number alterations were obvious given the variation in green and red colorization in the hybridization, quantitative fluorescence analysis of digital 
images was required for accurate CGH analysis (Raap, 1998). Image acquisition and analysis were performed on a Leica Q550CW cytogenetics workstation, which included a LEICA DM-RXA fluorescent microscope (Fig. 15) (Leica Inc., Exton, PA), and a SenSys cooled CCD camera (Photometrics Ltd., Tuscon, AZ). A CCD camera was mounted on the microscope for image capture and import into the software. DAPI, FITC, and TRITC images were acquired separately and combined to create a CGH image of each metaphase spread suitable for analysis in the Leica QCGH software (Fig. 16). Each image was visualized using an appropriate single bandpass filter, thus there was limited overlap in excitation and emission spectra (Joos et al., 1994; du Manoir et al., 1995a). Inverting the DAPI image approximated G-banding for accurate karyotyping of each metaphase spread (Fig. 17) (Trask, 1991). Compilation of CGH ratio profiles from a minimum of 10 metaphase spreads per cell line, allowed the generation of mean ratio profiles in the form of green:red (test:reference DNA) fluorescence ratios (Fig. 18). Ninety-five percent confidence intervals were applied to each of the collective means. The copy number thresholds for gain and loss were 0.2 units above or below the midline (1.2 and 0.8). While significant gains and losses were observed on the sex chromosomes, information on the sex of the parent tumor was not available for all cell lines (Table 1). Therefore, our cell line analysis considered only the autosomes.

\section{SPECTRAL KARYOTYPING.}

SKY was performed using the SkyPaint ${ }^{\mathrm{TM}}$ hybridization kit (Applied Spectral Imaging, Inc., Carlsbad, CA) and results were visualized using the SkyVision ${ }^{\mathrm{TM}}$ spectral imaging system (Fig. 9) (Applied Spectral Imaging, Inc., Carlsbad, CA). Metaphase spreads were prepared from each of the uveal melanoma cell lines via conventional 


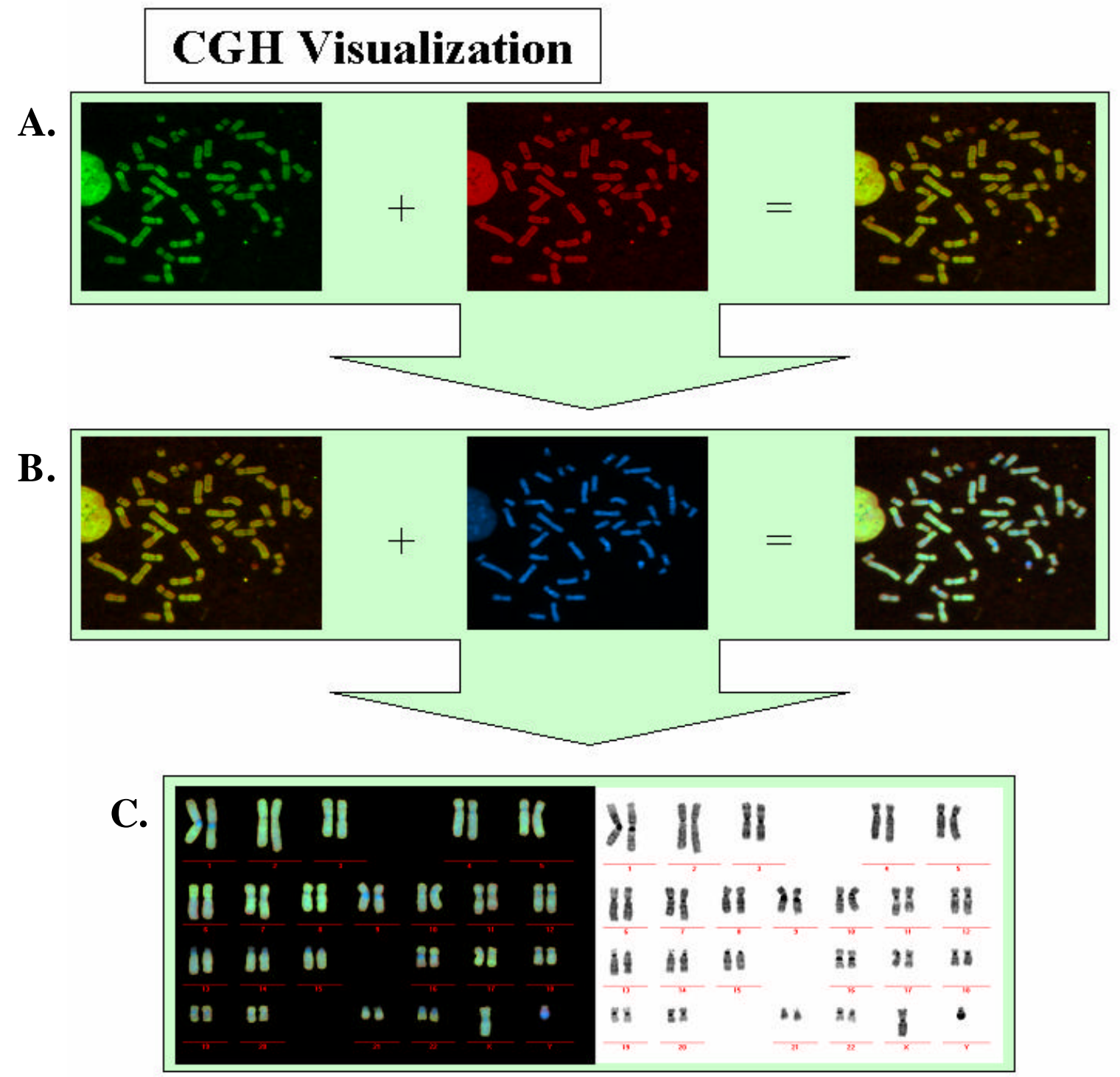

Figure 15. Step-wise Process of CGH Visualization. (A) The captured FITC and TRITC images are combined to form a single overlay image. (B) This image is combined with the DAPI counterstain to yield a completed CGH image. (C) The resultant image from (B) is karyotyped. In the completed color karyotype, similar variations in the amount of bound test probe are seen in homologous chromosome pairings. See Figure 16 for additional detail regarding image capture. 

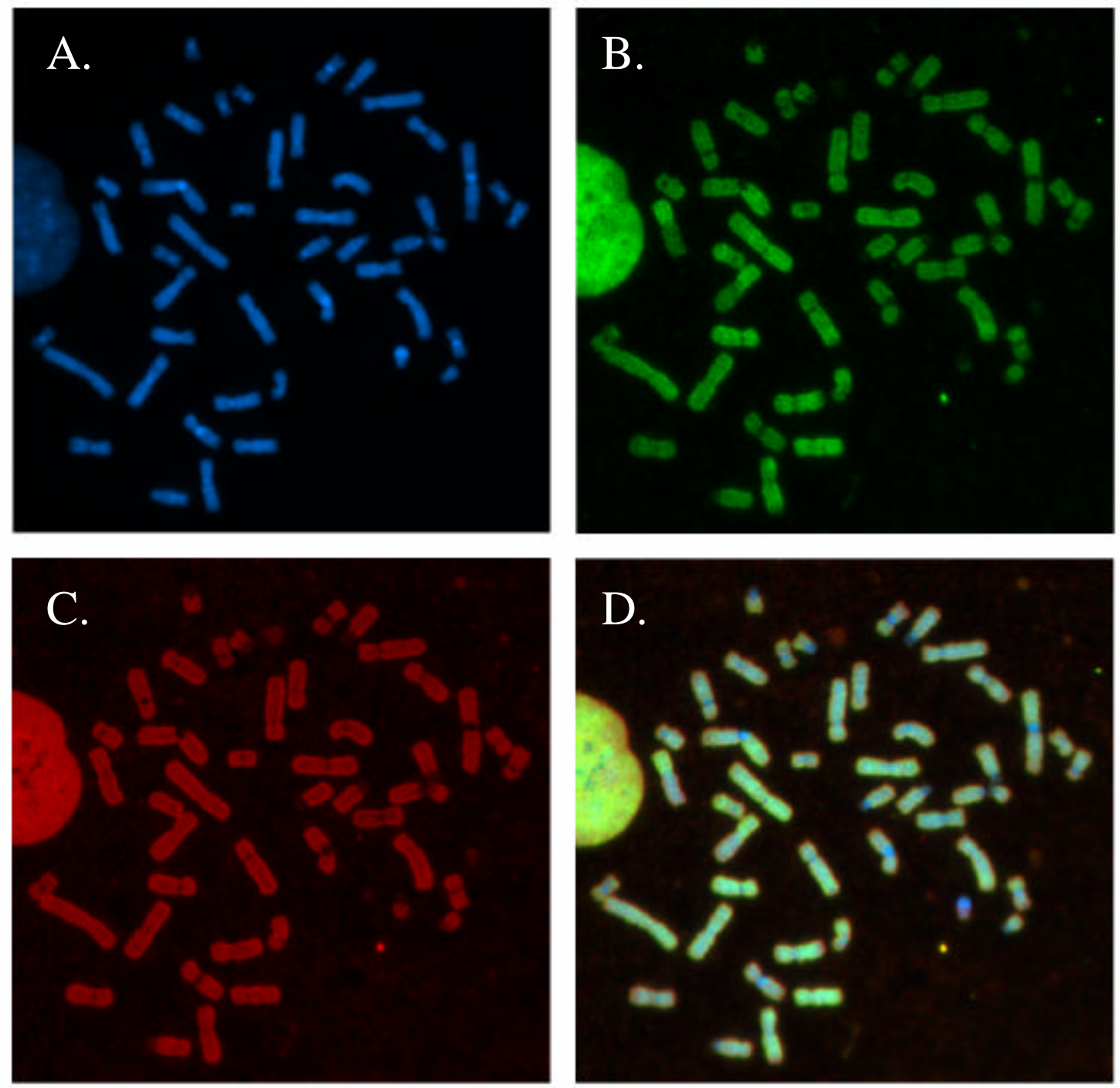

Figure 16. Image Capture for CGH Analysis. Each of these images is the identical normal male metaphase spread detected with three different fluorescent filters. (A) The metaphase chromosomes counterstained with 4',6-diamidino-2-phenylindole (DAPI) (Sigma Co., St. Louis, MO). Biotinylated tumor DNA signals were detected with avidin-conjugated fluorescein isothiocyanate (FITC) (Vector Labs, Burlingame, CA), and digoxigenin-11dUTP-labelled normal DNA was visualized using tetramethylrhodamine is othiocyanate (TRITC) conjugated antibodies (Sigma Co., St. Louis, MO). (B) The FITC image, and (C) the TRITC image. These three fluorescent images (A, B and $\mathbf{C})$ combine to form (D) the completed CGH image. This newly combined image is suitable for CGH analysis; the image is classified and karyotyped, followed by the generation of $\mathrm{CGH}$ profiles to illustrate the amplifications and deletions present within the tumor genome. All image acquisition was performed using a Leica Q550CW cytogenetics workstation which includes a Leica DMRXA fluorescent microscope (Leica Inc., Exton, PA) and a SenSys cooled CCD camera (Photometrics Ltd., Tuscon, AZ). 

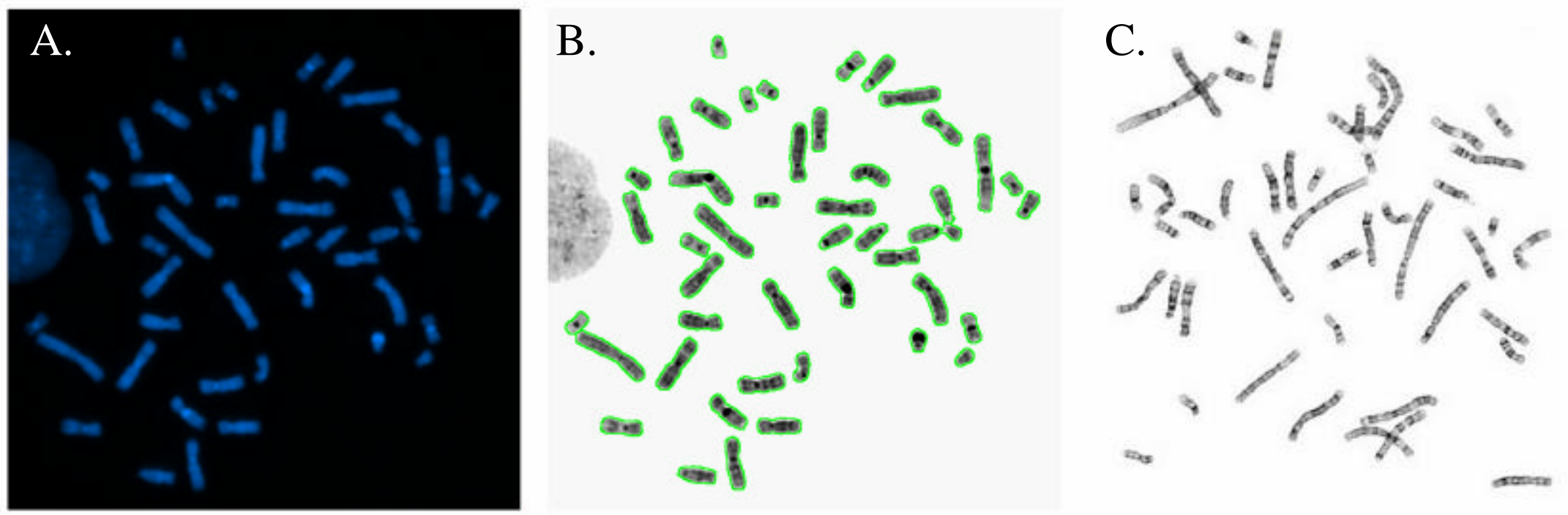

Figure 17. DNA Counterstain. (A) The counterstained metaphase spread stained with DAPI. (B) The inversion of the DAPI counterstain yields banding patterns comparable to G-banding, and allows for accurate karyotyping of the metaphase spread chromosomes. (C) For comparison purposes, a Gbanded image from a different metaphase spread.

Image (C) was adapted from Genetics of Cancer Resource Center, http://www.intouchlive.com/cancergenetics/chrm-anl.html. 


\section{Image Processing}

A.

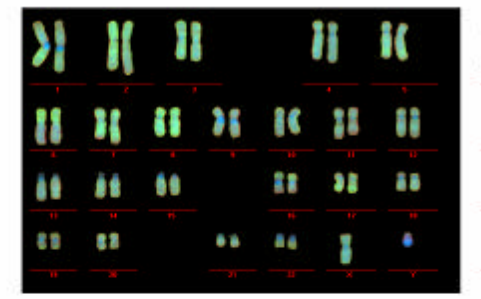

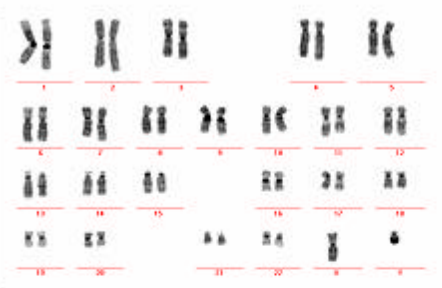

\section{B.}
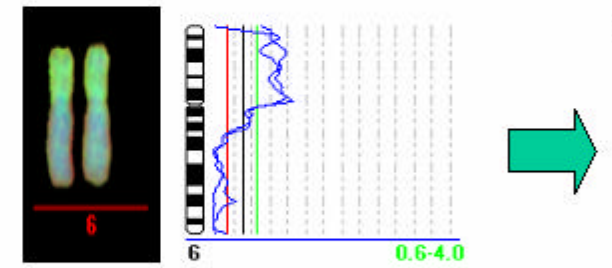

Red:Green Ratio Profile

C.

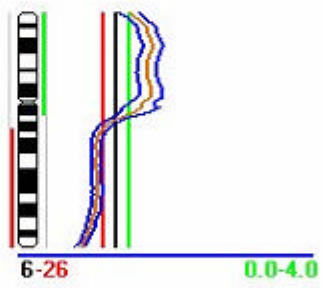

Mean Profile w/ 95\% Confidence Interval

\section{Image Analysis}

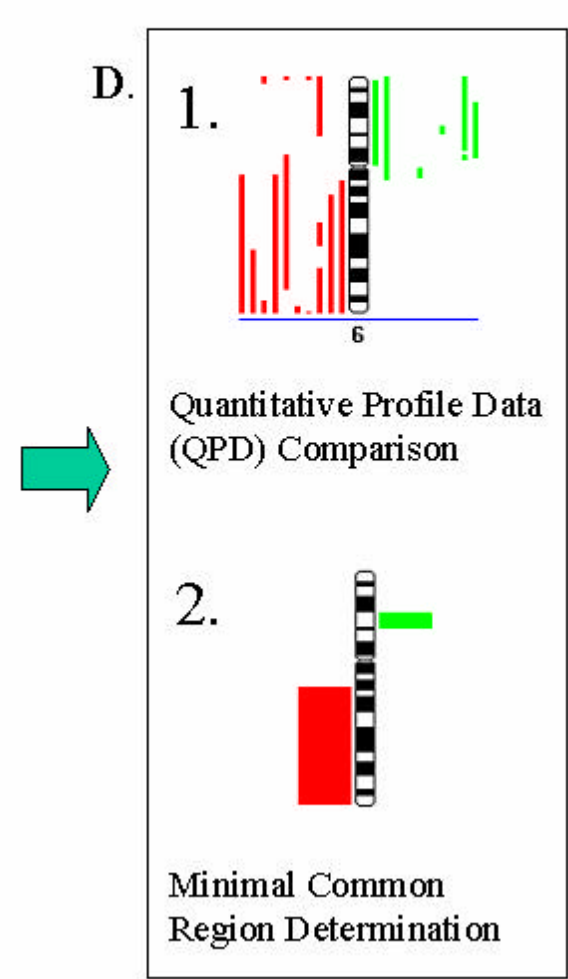

Figure 18. A Schematic for the Process of CGH Analysis. In (A), the completed karyoptype from Figure 12 results in a (B) fluorescence ratio profile (red:green for reference:test), where each homologue has its own profile tracing as seen in blue. In (C), compilation of a minimum of 10 ratio profiles comprises the mean ratio profile, in which $95 \%$ confidence intervals are assigned to the data. Also shown are the gain (green) and loss (red) bars along the length of the ideogram, which correspond to the mean gains and losses in the test sample. In (D), the side-by-side comparison of all sample mean profiles results in a QPD comparison (1) and the derivation of minimal common regions (2) from the comparison of means. 
cytogenetic techniques as described previously. Fresh slides were aged at room temperature for a week and then hybridized with SkyPaint ${ }^{\mathrm{TM}}$ probes as per the manufacturer's instructions. Selected metaphase slides were treated with $6 \mu 1$ pepsin stock solution in $50 \mathrm{ml}$ of $0.01 \mathrm{M} \mathrm{HCl}$ for 5 minutes, washed twice in $1 \times \mathrm{PBS}$ (w/o $\mathrm{MgCh}$ or $\mathrm{CaCl}_{2}$ ) for 5 minutes, once in $1 \mathrm{xPBS} / \mathrm{MgCb}$, and then dehydrated in a series of ethanol dilutions $(70 \%, 85 \%$, and $100 \%)$ for 2 minutes each. The SkyPaint ${ }^{\mathrm{TM}}$ probe mixture was denatured by incubation at $80^{\circ} \mathrm{C}$ for 7 minutes followed by a pre-anneal incubation at $37^{\circ} \mathrm{C}$ for 1 hour. Pretreated slides were denatured by incubation with $100 \mu 170 \%$ FA/SSC for at $80^{\circ} \mathrm{C}$ for 1.5 minutes. Ten microliters of denatured, pre-annealed probe was applied to the denatured, pretreated slides, a coverslip was placed atop the probe and sealed with rubber cement (Elmer's Products, Inc., Columbus, OH) prior to incubation at $37^{\circ} \mathrm{C}$ for 2 nights. On the third day the rubber cement seals and coverslips were removed. The slides were washed once in $50 \% \mathrm{FA} / \mathrm{SSC}$ at $45^{\circ} \mathrm{C}$ for 5 minutes, twice in $1 \mathrm{xSSC}$ (see appendix) at $45^{\circ} \mathrm{C}$ for 5 minutes each, and finally in $4 \mathrm{xSSC} / 0.1 \%$ Tween 20 at $45^{\circ} \mathrm{C}$ for 2 minutes. Eighty microliters of the manufacturer-supplied blocking reagent was applied, the slides were coverslipped, and incubated at $37^{\circ} \mathrm{C}$ for 30 minutes. Following the blocking step, the coverslips were removed and $80 \mu 1$ antibodies, supplied as buffer 1 (Applied Spectral Imaging, Inc., Carlsbad, CA), were applied. Slides were incubated at $37^{\circ} \mathrm{C}$ for 45 minutes followed by 3 washes in $4 \mathrm{xSSC} / 0.1 \%$ Tween 20 at $45^{\circ} \mathrm{C}$ for 3 minutes each. Eighty microliters of a second set of antibodies, supplied as buffer 2 (Applied Spectral Imaging, Inc., Carlsbad, CA), were applied and the slides were incubated at $37^{\circ} \mathrm{C}$ for 45 minutes. Following this incubation, the slides were washed 3 times in $4 \times \mathrm{xSC} / 0.1 \%$ Tween 20 at $45^{\circ} \mathrm{C}$ for 3 minutes each and once in $\mathrm{dH}_{2} \mathrm{O}$ for 2 
minutes. The slides were allowed to dry, $20 \mu 1$ of manufacturer-supplied DAPI/antifade solution was applied, and a coverslip was mounted over each slide. A minimum of 3 metaphase spreads per cell line were captured and analyzed using the SpectraCube ${ }^{\circledR}$ and a SkyVision $^{\mathrm{TM}}$ workstation. While CGH data is sored in the form of CCD images captured through various filters, data from a spectral image is captured in a single exposure and stored in three dimensions, the $\mathrm{x}$ and $\mathrm{y}$ planes and the integration of light intensity on a wavelength scale, hence the use of the term "cube" in SpectraCube ${ }^{\circledR}$ (Rothmann et al., 1998).

\section{INTERPHASE FLUORESCENCE IN SITU HYBRIDIZATION (FISH).}

Interphase FISH was performed on uveal melanoma cell lines, created via metaphase chromosome preparation techniques, using human chromosome 3-specific centromere (3cen) probe (Cambio Ltd., Cambridge, UK). Application of the slide pretreatment protocol for CGH prior to hybridization removed any RNA, cytoplasm, or proteins complexed with the DNA (Kallioniemi et al., 1992). The protocol consisted of treating the normal male and cell line slide preparations with RNase A, pepsin/ $\mathrm{HCl}$, and $1 \%$ formaldehyde in $1 \times \mathrm{PBS} / \mathrm{MgCh}$, and lastly dehydration in a series of ethanols $(70 \%$, $85 \%$, and $100 \%$ ), prior to denaturation and hybridization. The hybridization protocol for the 3cen probes was a slight modification of the manufacturer's instructions for optimization. Pretreated slides were denatured in $70 \% \mathrm{FA} / \mathrm{SSC}$ at $70^{\circ} \mathrm{C}$ for 5 minutes, dehydrated in a series of ethanols and allowed to air dry on a $37^{\circ} \mathrm{C}$ slide warmer. Denaturation of $20 \mu 1$ of indirectly-labeled (biotinylated) 3 cen probe at $75^{\circ} \mathrm{C}$ for 5 minutes occurred in parallel with slide denaturation and dehydration. The probe was 
applied to the slide, sealed under a $22 \mathrm{~mm}^{2}$ glass coverslip with Elmer's rubber cement, and allowed to hybridize for 16 hours at $37^{\circ} \mathrm{C}$.

Following hybridization, the coverslips were removed, and the slides washed twice in $45^{\circ} \mathrm{C} 50 \% \mathrm{FA} / \mathrm{SSC}$ for 5 minutes, and then twice in $45^{\circ} \mathrm{C} 0.4 \mathrm{xSSC}$ for 5 minutes. The slides were dipped in $45^{\circ} \mathrm{C} 4 \mathrm{xSSC} / 0.05 \%$ Tween 20 (see appendix), incubated for 1 hour at $37^{\circ} \mathrm{C}$ with $150 \mu \mathrm{l} 3 \%$ non-fat dried milk (NFDM) blocking solution (see appendix), and then dipped in 4xSSC/0.05\% Tween 20 a second time. Each slide had 100-200 $\mu$ l of a given antibody solution layered onto it, and slides were incubated for 1 hour with each of 3 separate antibody layers. The first layer of antibodies consisted of avidin-FITC and avidin-Rhodamine in 3\% NFDM, the second layer was biotinylated anti-avidin, and the third layer was identical to the first. Following each antibody layer, slides were washed 3 times for 5 minutes each in $45^{\circ} \mathrm{C}$ 4xSSC/0.05\%Tween 20. After the final wash, slides were counterstained with DAPI, rinsed with $\mathrm{dH}_{2} \mathrm{O}$ for 5 minutes, and dehydrated in a series of ethanols $(70 \%, 85 \%$, and $100 \%$ ) for 3 minutes each. Finally, a glass coverslip was mounted over an antifade solution to complete each of the slides. Visualization of the duatcolored probe was with the Leica Q550CW cytogenetics workstation, as described for CGH. FITC, TRITC, and DAPI images were acquired separately and combined to view signal localization and score signal counts within the nuclei. Two hundred nuclei per cell line were scored for 3cen signals. We closely adhered to the rules for analysis of signal distribution as set out by Herrington and colleagues, and the criteria for interphase FISH as described by Hopman et al. (Hopman et al., 1988; Herrington et al., 1995). 
Interphase FISH was performed on uveal melanoma cell lines using directlylabeled telomere probes for 3p (FITC) and 3q (Texas Red) (Cytocell Ltd., Banbury, Oxfordshire, UK). Slide pretreatment was identical to that described for 3cen FISH. Tandem hybridization of the telomere- 3 probes followed the manufacturer's instructions, except for the substitution of a $22 \mathrm{~mm}^{2}$ glass coverslip. Codenaturation of the probe and slide on a Vysis Hybrite block (Vysis Inc., Downer's Grove, IL), sealed together under a $22 \mathrm{~mm}^{2}$ glass coverslip, preceeded overnight hybridization in a humidified chamber at $37^{\circ} \mathrm{C}$. Slides were treated using the manufacturer's post-hybridization protocol, stained with DAPI and mounted with antifade as described above. Again, 200 nuclei per cell line were scored for $3 p$ and $3 q$ signals, and published criteria for interphase FISH and guidelines for analysis of signal distribution were adopted (Hopman et al., 1988; Herrington et al., 1995). Image capture was identical to that described for the 3cen hybridization.

Hardware requirements for FISH analysis are similar to those described for other molecular cytogenetic techniques. For our purposes, the hardware requirements described for $\mathrm{CGH}$ digital imaging microscopy were sufficient. Hybridized slides were scored using a Leica Q550CW cytogenetics workstation, which includes a LEICA DMRXA fluorescent mic roscope (Leica Inc., Exton, PA), and a SenSys cooled CCD camera (Photometrics Ltd., Tuscon, AZ). Use of a CCD camera is the most sensitive method for obtaining high quality digital fluorescence images (Joos et al., 1994).

\section{Statistical ANALYSES.}

Extensive quantitative manipulations of the data from the cell line study were necessary in order to compare the results from CGH, SKY and FISH analyses. In the 
tumor study, statistical analyses were used in order to isolate regions of prognostic significance.

\section{Cell Line CGH Analysis.}

To effectively malipulate the $\mathrm{CGH}$ data from the cell line study, a pixel quantification of net area under each ratio profile curve was applied using a Visual Basic v. 6.0 (Microsoft Corp., Redmond, WA) program created for Image Pro Plus v. 4.5 (Media Cybernetics, Silver Spring, MD) for each of the chromosomes in a given cell line. The intent was to extrapolate the extent of genomic imbalance represented by each of the profiles contributing to the mean. In other words, analyses were made to determine the quantity of shift from the midline of the profile represented in the $\mathrm{CGH}$ data given the addition or subtraction of the amount of chromosomal material shown to be gained or lost for a given chromosome. Analysis of individual profiles rather than the mean profiles was determined to be the most accurate approach, similar to an approach previously applied to pseudodiploid and pseudotriploid test samples (Rosenberg et al., 1997). The following formula was applied to calculate relative balance (RB) represented in each individual chromosome profile in all metaphase spreads analyzed for each cell line:

\section{CGH RB = (NA/PL x 1/len1 $)+1$}

For a given chromosome, NA was the number of pixels under ratio profile to the right of the midline minus the number of pixels under the ratio profile to the left of the midline, PL was the vertical profile pixel length, in pixels, and len1 was the distance, in pixels, representing a unit change in copy number on the x-axis of the ratio profile. CGH RB, the extrapolated ratio value used as a measure of balance, was determined for a specific 
chromosome in any given cell for each of the cell lines. This allowed a quantification of the expected amount of genomic imbalance relative to the copy number ratio.

\section{Cell Line SKY Analysis.}

A pixel quantification of each of the 24 chromosome classification colors in SKY was applied using a Visual Basic v. 6.0 (Microsoft Corp., Redmond, WA) program written for Image Pro Plus v. 4.5 (Media Cybernetics, Silver Spring, MD). This quantification was performed for each of the available spreads from the 10 cell lines, and normalized against representative whole chromosomes from within the same spread. The relative chromosome equivalent (RCE) feature was calculated for each of the 22 autosomes from the SKY classification images according to the formula:

\section{SKY RCE $=$ PT/PN}

For a metaphase spread, PT was the number of pixels designated for a given chromosome throughout the SKY classification image, and PN was the number of pixels assigned to all cytogenetically normal chromosomes of the same classification divided by the number of normal chromosomes counted. This internal normalization resulted in determination of relative chromosome equivalents for each cell line. Using this method, the relative amount of genomic imbalance could be compared between chromosomes from a given cell line, but SKY RCE remained an absolute measure of chromosomal material and therefore was directly comparable with the FISH results.

A relative genomic balance for the SKY data (SKY RB) was calculated for each of the 22 autosomes by dividing the individual chromosome SKY RCE values by the mean SKY RCE (comparable to CGH midline) for all chromosome types. The SKY data, an absolute measure in its original form, becomes transformed into a ratio and was 
thereby compensated for ploidy but not aneusomy. SKY RCE and SKY RB results for OCM-3 were not reported due to hardware failure while archiving SKY data.

\section{Cell Line FISH Analysis.}

A FISH relative chromosome equivalent (FISH RCE) feature was calculated for chromosome 3 in each cell line, as the sum of all 3cen, $3 p$ and $3 q$ hybridization signals (200 nuclei scored for each) divided by the total number of signals (divided by 600). This was essentially a combined average for the individual signal means. FISH RCE remained an absolute measure of chromosome 3 copy number, and was most readily compared with the SKY RCE. Since there was no available compensation for genomic ploidy, because FISH revealed only chromosome 3 copy number, determination of FISH $\mathrm{RB}$ was not reasonable.

\section{Tumor CGH Analysis.}

Minimal common regions were generated from mean ratio profiles of 82 archival FFPE tumor specimens analyzed by $\mathrm{CGH}$, for those chromosomal regions evidencing gains or losses in greater than $30 \%$ and less than $70 \%$ of the cases. Minimal common regions were further subdivided by gender, for accurate analysis of copy number imabalnces on the sex chromosomes, and survival, to determine which chromosomal regions were potentially of value in prognosis.

CGH data for individual cases was recorded as normal (1), gain (2), loss (0), or both ( 0 and 2$)$ at each minor band for all 24 chromosomes in the mean ratio profiles. Minor band data was collated for each major band, and major bands were initially scanned for significant gains or losses with a two-tailed t-test. Only those chromosomal regions shown to be significant in the analysis of major bands were then analyzed at the 
level of the minor band, also with a two-tailed ttest. Individual minor bands with univariate significance following that analysis were compared with cell type, tumor size, mean of the ten largest nucleoli, and survival in a Cox Regression analysis, to determine which chromosomal regions correlate most strongly with prognosis. Univariate KaplanMeier cause specific survival curves were generated for those regions determined to have prognostic significance in the Cox Regression analysis. Multivariate predictors of prognosis were also explored, as first described by McLean and colleagues, and multivariate Kaplan-Meier cause specific survival curves were also generated (McLean et al., 1977). 


\section{RESULTS}

\section{UVEal Melanoma Cell Lines.}

\section{Comparative Genomic Hybridization.}

Mean profiles for all 10 cell lines were compared (Fig. 19). Copy number abnormalities identified in greater than $50 \%$ of the mean ratio profiles comprise the minimal common regions, which were pinpointed based upon the most recent chromosome ideogram and genome mapping data available (Fig. 20).

Many of these abnormalities aligned with previously published findings, including $\mathrm{CGH}$ and classical cytogenetic data (Tables 2 and 3). Minimal common regions were used to identify genes likely to be involved in uveal melanoma (Table 4). Many of these genes have either been previously linked to uveal melanoma or its predisposing factors and/or are involved in cell-cycle control.

Gains and losses often consisted of large portions of chromosomes, and in some cases involved whole chromosomes. Whole chromosome gains were observed in individual cell lines: chromosome 7 (OMM-1), chromosome 8 (MEL270) and chromosome 17 (OCM-3). Whole chromosome gains were observed in 3 cell lines for chromosomes 20 (OCM-3, OCM-8, and OMM-1) and 22 (OCM-3, OCM-8, and OMM2.3). The greatest gains of chromosome 3 were evidenced in OCM-1, OM431, and OMM-2.3. Only OCM-1 and OM431 had gains that included the centromere. Other significant p- and q-arm gains were seen in OCM-3 and OCM-8. A whole chromosome loss was observed in one instance on chromosome 4 (OCM-3).

The frequent involvement of chromosomes 3, 6 and 8 in copy number imbalances was apparent (Figs. 19 and 20). There were multiple consistent gains along chromosome 

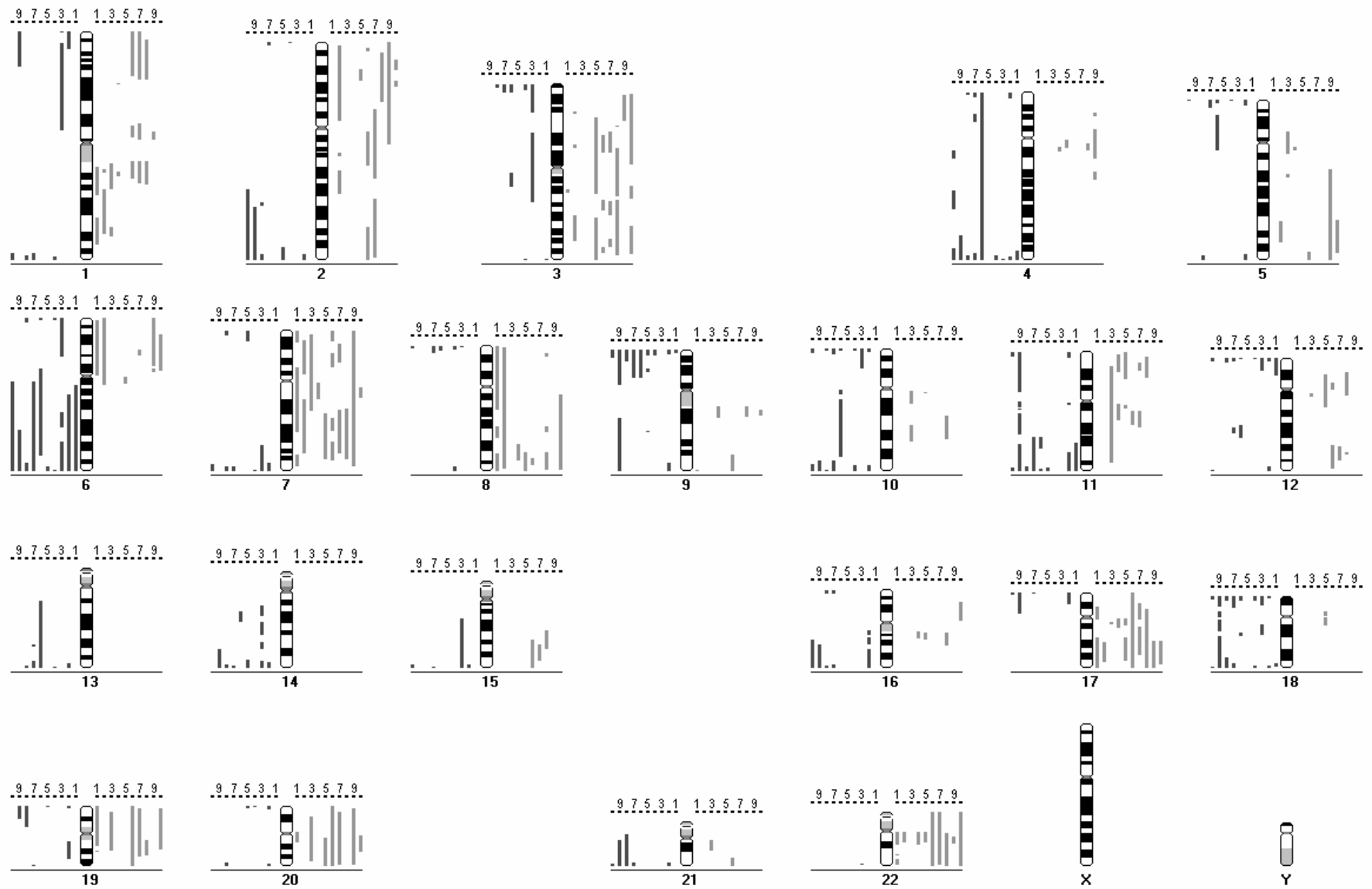

Figure 19. Quantitative Comparison Data for Genomic Imbalances in Ten Cell Lines. The gain threshold was set at a 1.2 copy number ratio, and the loss threshold was set at a 0.8 copy number ratio. Light gray bars to the right of the ideogram represent gains in a specific chromosomal region for each cell line, and dark gray bars to the left of each ideogram represent regions of loss in the cell line genome. The gain and loss bars are paired by cell line, starting with the pair closest to the ideogram, and then flanking outward. A scale with the numeric code for each cell line, as designated in Table 1, is provided above each ideogram: 1 = 92-1, 2 = MEL270, $3=$ MEL285, $4=$ MEL290, $5=$ OCM-1, $6=$ OCM-3, $7=$ OCM-8, $8=$ OM431, $9=$ OMM1, and $10=$ OMM2.3 Only autosomes are considered in our analysis. 

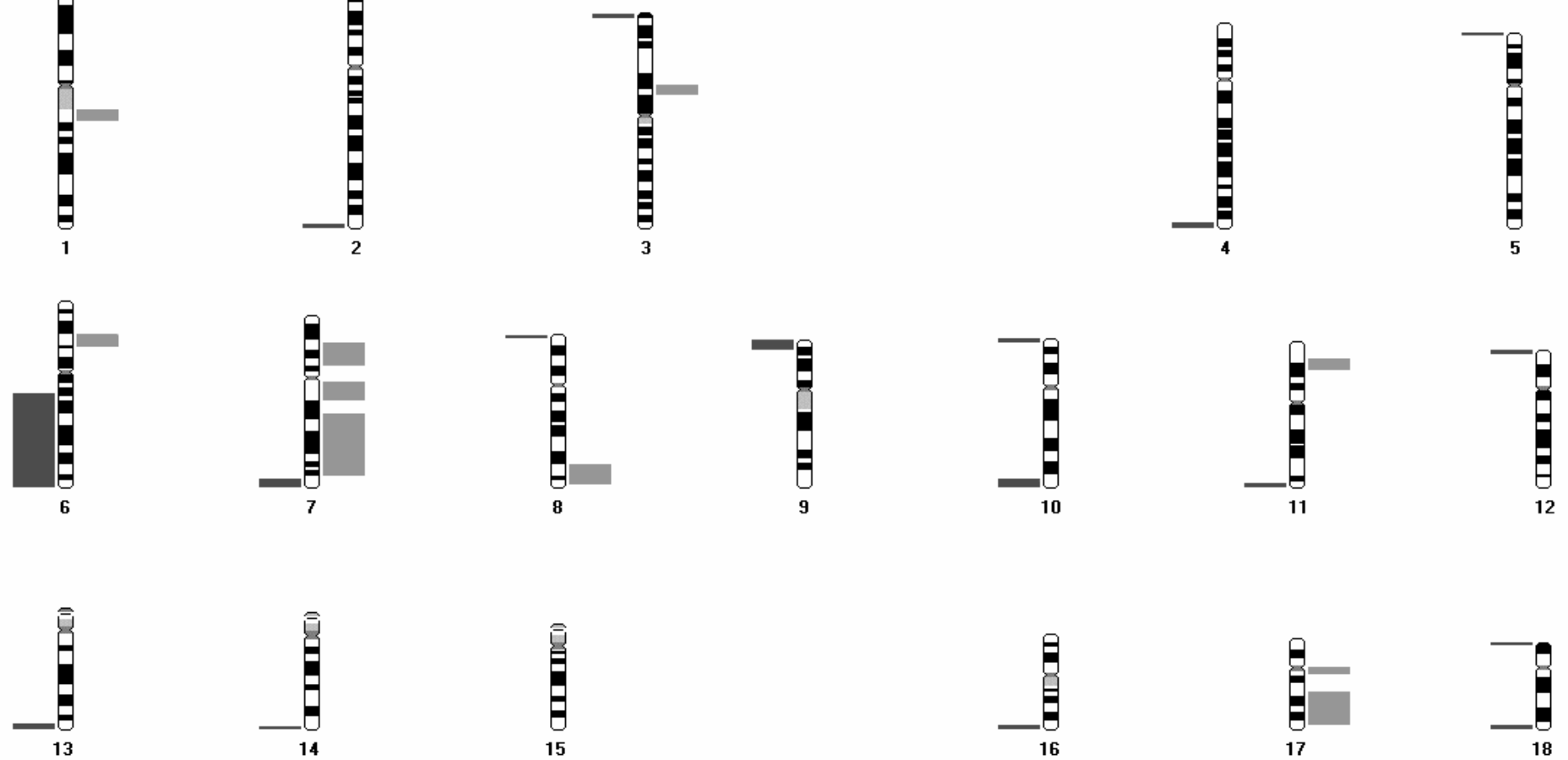

夏
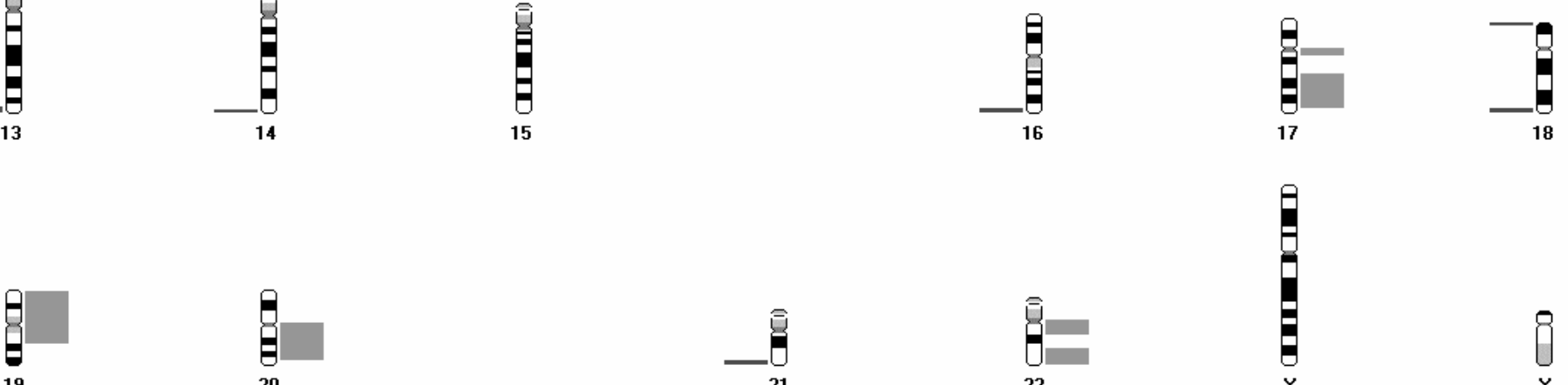

16
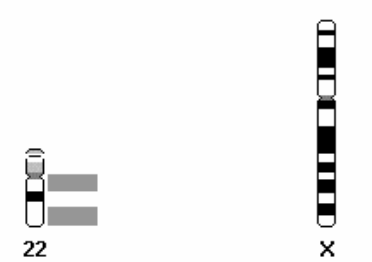

$\bigoplus_{Y}^{8}$

Figure 20. Minimal Common Regions of Gain and Loss Distilled from CGH Mean Profile Data of Ten Uveal Melanoma Cell Lines. The light gray bars to the right of the ideogram represent gain, and dark gray bars to the left of the ideogram represent regions of loss, common among at least $50 \%$ of the cell lines. These minimal common regions may denote chromosomal regions related to the development and progression of uveal melanoma. Only autosomes are considered in our analysis. 
Table 2. A Comparison of Gains Seen in Ten Uveal Melanoma Cell Lines Compared with Previously Reported Chromosomal Abnormalities in Uveal Melanoma.

\begin{tabular}{|c|c|c|c|c|c|c|c|c|}
\hline \multicolumn{2}{|c|}{$\begin{array}{l}\text { Gains Observed in the Current } \\
\text { Study }\end{array}$} & \multirow{2}{*}{$\begin{array}{c}\text { Cell Lines }^{\mathbf{b}} \\
1,2,3,6,7,8 \\
1,3,4,6,7,8\end{array}$} & \multicolumn{2}{|c|}{ Gains Reported by CGH $^{\mathrm{a}}$} & \multirow[t]{2}{*}{ Reference } & \multicolumn{2}{|c|}{$\begin{array}{l}\text { Gains Reported by } \\
\text { Classical Cytogenetics }\end{array}$} & \multirow[t]{2}{*}{ Reference } \\
\hline $\begin{array}{c}1 \mathrm{q} 21.1-\mathrm{q} 21.2 \\
1 \mathrm{q} 21.2\end{array}$ & $\begin{array}{l}(60 \%) \\
(60 \%)\end{array}$ & & $1 \mathrm{q}$ & $(9.1 \%)$ & & & & \\
\hline $3 \mathrm{p} 13-\mathrm{p} 14.1$ & $(50 \%)$ & $2,5,6,7,10$ & & & & & & \\
\hline $6 \mathrm{p} 21.2-\mathrm{p} 21.3$ & $(50 \%)$ & $1,2,7,9,10$ & $\begin{array}{c}6 p \\
6 p \\
6 p \\
6 p \\
6 p 21-\text { pter }\end{array}$ & $\begin{array}{l}(45.5 \%) \\
(57 \%) \\
(42 \%) \\
(40 \%) \\
(54.5 \%)\end{array}$ & $\begin{array}{c}\text { Speicher et al., } 1994 \\
\text { Ghazvini et al., } 1996 \\
\text { " } \\
\text { Gordon et al., } 1994 \\
\text { Speicher } \text { et al., } 1994\end{array}$ & $\begin{array}{c}6 p \\
6 p \\
6 p 12-6 p t e r\end{array}$ & $\begin{array}{c}(46.3 \%) \\
(25 \%) \\
(25 \%)\end{array}$ & $\begin{array}{c}\text { White } \text { et al., } 1998 \mathrm{~b} \\
\text { Wiltshire } \text { et al., } 1993 \\
\text { " }\end{array}$ \\
\hline $7 \mathrm{p} 13-\mathrm{p} 15.2$ & $(50 \%)$ & $2,3,6,7,9$ & & & & & & \\
\hline $\begin{array}{c}7 \mathrm{q} 11.21 \text { - q11.23 } \\
7 \mathrm{q} 21.2 \text { - q21.3 } \\
7 \mathrm{q} 21.3 \text { - q22.1 } \\
7 \mathrm{q} 22.1 \text { - q31.1 } \\
7 \mathrm{q} 31.1 \text { - q31.3 } \\
7 \mathrm{q} 31.3 \text { - q33 } \\
7 \mathrm{q} 34-\mathrm{q} 35\end{array}$ & $\begin{array}{l}(50 \%) \\
(60 \%) \\
(60 \%) \\
(70 \%) \\
(50 \%) \\
(60 \%) \\
(50 \%)\end{array}$ & $\begin{array}{c}1,3,4,9,10 \\
1,2,3,7,8,9 \\
1,2,5,7,8,9 \\
1,2,5,6,7,8,9 \\
2,5,7,8,9 \\
2,5,6,7,8,9 \\
1,5,6,8,9\end{array}$ & $7 \mathrm{q}$ & $(70 \%)$ & Gordon et al., 1994 & & & \\
\hline $\begin{array}{c}8 \mathrm{q} 24.1-\mathrm{q} 24.2 \\
8 \mathrm{q} 24.2-\mathrm{q} 24.3 \\
8 \mathrm{q} 24.3\end{array}$ & $\begin{array}{l}(60 \%) \\
(60 \%) \\
(60 \%)\end{array}$ & $\begin{array}{c}1,2,4,5,8,10 \\
1,2,5,6,8,10 \\
1,2,5,8,10\end{array}$ & $\begin{array}{c}8 q \\
8 q \\
8 q \\
8 q \\
8 q 24-\text { qter }\end{array}$ & $\begin{array}{c}(54.5 \%) \\
(100 \%, \text { fresh }) \\
(57 \%, \text { fixed }) \\
(100 \%) \\
(63.6 \%)\end{array}$ & $\begin{array}{c}\text { Speicher et al., } 1994 \\
\text { Ghazvini et al., } 1996 \\
\text { " } \\
\text { Gordon et al., } 1994 \\
\text { Speicher et al., } 1994\end{array}$ & $\begin{array}{c}8 q \\
8 \\
8 q \\
8 q 13.1-8 q \text { ter }\end{array}$ & $\begin{array}{l}(48.1 \%) \\
(25 \%) \\
(50 \%) \\
(25 \%)\end{array}$ & $\begin{array}{c}\text { White } \text { et al., } 1998 \mathrm{~b} \\
\text { Wiltshire } \text { et al., } 1993 \\
" \\
"\end{array}$ \\
\hline $11 \mathrm{p} 14-\mathrm{p} 15.1$ & $(50 \%)$ & $3,4,5,7,8$ & $11 \mathrm{p}$ & $(9.1 \%)$ & $"$ & & & \\
\hline
\end{tabular}


Table 2 (cont'd). A Comparison of Gains Seen in Ten Uveal Melanoma Cell Lines Compared with Previously Reported Chromosomal Abnormalities in Uveal Melanoma.

\begin{tabular}{|c|c|c|c|c|c|c|c|}
\hline \multicolumn{2}{|c|}{$\begin{array}{l}\text { Gains Observed in the Current } \\
\text { Study }^{\mathrm{a}}\end{array}$} & \multirow{2}{*}{$\begin{array}{c}\text { Cell Lines }^{\mathbf{b}} \\
3,4,5,6,8\end{array}$} & \multicolumn{2}{|c|}{ Gains Reported by $\mathbf{C G H}^{\mathrm{a}}$} & \multirow{2}{*}{$\begin{array}{c}\text { Reference } \\
\text { Speicher } \text { et al., } 1994\end{array}$} & \multirow[t]{2}{*}{$\begin{array}{c}\text { Gains Reported by } \\
\text { Classical Cytogenetics }\end{array}$} & \multirow[t]{2}{*}{ Reference } \\
\hline $17 q 11.1-q 11.2$ & $(50 \%)$ & & 17 & $(18.2 \%)$ & & & \\
\hline $17 q 21.3-q 22$ & $(50 \%)$ & $1,5,6,7,8$ & & & & & \\
\hline $17 q 22-q 24$ & $(80 \%)$ & $1,2,5,6,7,8,9,10$ & & & & & \\
\hline $17 q 24$ & $(70 \%)$ & $1,2,5,6,8,9,10$ & & & & & \\
\hline $17 q 24-q 25$ & $(60 \%)$ & $1,2,6,8,9,10$ & & & & & \\
\hline $19 \mathrm{p} 13.3-\mathrm{q} 12$ & $(50 \%)$ & $1,3,6,7,10$ & & & & & \\
\hline $19 \mathrm{q} 12-\mathrm{q} 13.1$ & $(60 \%)$ & $1,3,6,7,8,10$ & & & & & \\
\hline $19 q 13.1-q 13.2$ & $(50 \%)$ & $3,6,7,8,10$ & & & & & \\
\hline 20p11.1 - q11.1 & $(50 \%)$ & $1,3,6,7,9$ & 20 & $(14 \%)$ & Ghazvini et al., 1996 & & \\
\hline $20 \mathrm{q} 11.1-\mathrm{q} 11.2$ & $(70 \%)$ & $1,3,5,6,7,9,10$ & & & & & \\
\hline 20q11.2 - q13.1 & $(60 \%)$ & $3,5,6,7,9,10$ & & & & & \\
\hline $20 q 13.1$ - q13.3 & $(50 \%)$ & $3,5,6,7,9$ & & & & & \\
\hline $22 \mathrm{q} 11.1-\mathrm{q} 11.2$ & $(80 \%)$ & $1,2,4,5,6,7,8,10$ & 22 & $(9.1 \%)$ & Speicher et al., 1994 & & \\
\hline $22 \mathrm{q} 11.2-\mathrm{q} 12.1$ & $(90 \%)$ & $1,2,4,5,6,7,8,9,10$ & $22 q$ & $(9.1 \%$, fresh $)$ & Ghazvini et al., 1996 & & \\
\hline $22 q 13.2$ & $(50 \%)$ & $1,6,7,8,10$ & $22 \mathrm{q}$ & $(42 \%$, fixed $)$ & & & \\
\hline $22 q 13.3$ - qter & $(50 \%)$ & $1,6,7,8,10$ & & & & & \\
\hline
\end{tabular}

${ }^{\mathrm{a}}$ The percentage in parentheses is the frequency of occurrence for a given gain.

${ }^{\mathrm{b}}$ See cell line key. 
Table 3. A Comparison of Losses Seen in Ten Uveal Melanoma Cell Lines Compared with Previously Reported Chromosomal Abnormalities in Uveal Melanoma.

\begin{tabular}{|c|c|c|c|c|c|c|c|c|}
\hline \multicolumn{2}{|c|}{$\begin{array}{l}\text { Losses Observed in the } \\
\text { Current Study }^{\mathrm{a}}\end{array}$} & \multirow{2}{*}{$\begin{array}{c}\text { Cell Lines }^{\mathbf{b}} \\
2,5,8,9,10\end{array}$} & \multicolumn{2}{|c|}{ Losses Reported by CGH } & \multirow[t]{2}{*}{ Reference } & \multicolumn{2}{|c|}{$\begin{array}{c}\text { Losses Reported by } \\
\text { Classical Cytogenetics }^{\text {a }}\end{array}$} & \multirow[t]{2}{*}{ Reference } \\
\hline 2q37.3 - qter & $(50 \%)$ & & & & & & & \\
\hline $3 \mathrm{p} 26-$ pter & $(50 \%)$ & $3,4,6,7,8$ & $\begin{array}{l}3 \\
3 \\
3 \\
3 \\
3\end{array}$ & $\begin{array}{c}(45.5 \%) \\
(57 \%, \text { fresh }) \\
(85 \%, \text { fixed }) \\
(70 \%) \\
(57 \%)\end{array}$ & $\begin{array}{l}\text { Speicher et al., } 1994 \\
\text { Ghazvini et al., } 1996 \\
\text { " } \\
\text { Gordon et al., } 1994 \\
\text { Prescher et al., } 1996\end{array}$ & $\begin{array}{l}3 \\
3 \\
3\end{array}$ & $\begin{array}{l}(80 \%) \\
(50 \%) \\
(100 \%)\end{array}$ & $\begin{array}{l}\text { White } \text { et al., 1998a } \\
\text { White } \text { et al., 1998b } \\
\text { Wiltshire } \text { et al., } 1993\end{array}$ \\
\hline $4 q 35$ - 4qter & $(90 \%)$ & $1,2,3,4,6,7,8,9,10$ & $4 q$ & $(14 \%)$ & Ghazvini et al., 1996 & & & \\
\hline 5 p15.3 - pter & $(50 \%)$ & $2,4,6,7,10$ & & & & & & \\
\hline $\begin{array}{c}6 \mathrm{q} 14-\mathrm{q} 21 \\
6 \mathrm{q} 21-\mathrm{q} 22.2 \\
6 \mathrm{q} 22.2-\mathrm{q} 23.3 \\
6 \mathrm{q} 23.3-\mathrm{q} 25.2 \\
6 \mathrm{q} 25.2-\mathrm{q} 26 \\
6 \mathrm{q} 26-\mathrm{q} 27 \\
6 \mathrm{q} 27-\mathrm{qter}\end{array}$ & $\begin{array}{l}(50 \%) \\
(60 \%) \\
(60 \%) \\
(70 \%) \\
(60 \%) \\
(70 \%) \\
(90 \%)\end{array}$ & $\begin{array}{c}1,2,6,7,10 \\
1,2,3,6,7,10 \\
1,2,6,7,9,10 \\
1,2,3,6,7,9,10 \\
1,2,3,7,9,10 \\
1,2,3,7,8,9,10 \\
1,2,3,4,5,7,8,9,10\end{array}$ & $\begin{array}{l}6 q \\
6 q \\
6 q\end{array}$ & $\begin{array}{c}(45.5 \%) \\
(42 \%, \text { fresh }) \\
(28 \%, \text { fixed })\end{array}$ & $\begin{array}{c}\text { Speicher et al., } 1994 \\
\text { Ghazvini et al., } 1996 \\
\text { " }\end{array}$ & $\begin{array}{c}6 q \\
6 q \\
6 q 12-6 q t e r\end{array}$ & $\begin{array}{l}(46.3 \%) \\
(25 \%) \\
(25 \%)\end{array}$ & $\begin{array}{c}\text { White } \text { et al., 1998b } \\
\text { Wiltshire } \text { et al., } 1993 \\
\text { " }\end{array}$ \\
\hline $7 q 36$ - qter & $(60 \%)$ & $2,3,4,7,8,10$ & & & & & & \\
\hline 8p23.3 - pter & $(50 \%)$ & $3,4,6,7,10$ & $8 p$ & $(28 \%)$ & $"$ & $8 \mathrm{p}$ & $(25 \%)$ & Wiltshire et al., 1993 \\
\hline $\begin{array}{l}9 \text { p23 - p24 } \\
9 \text { p24 - pter }\end{array}$ & $\begin{array}{l}(50 \%) \\
(90 \%)\end{array}$ & $\begin{array}{c}6,7,8,9,10 \\
1,2,4,5,6,7,8,9,10\end{array}$ & 9p21 - pter & $\begin{array}{c}(27.3 \%) \\
،\end{array}$ & 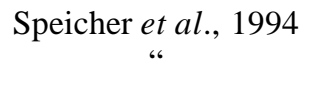 & $\begin{array}{c}9 p \\
9 p 23-9 \text { pter }\end{array}$ & $\begin{array}{l}(25 \%) \\
(25 \%)\end{array}$ & " \\
\hline $\begin{array}{l}10 \mathrm{p} 15.1 \text { - pter } \\
10 \mathrm{q} 26.2 \text { - qter }\end{array}$ & $\begin{array}{l}(70 \%) \\
(60 \%)\end{array}$ & $\begin{array}{c}2,3,4,6,7,8,10 \\
2,4,7,8,9,10\end{array}$ & & & & & & \\
\hline $11 \mathrm{q} 25$ - qter & $(80 \%)$ & $1,2,5,6,7,8,9,10$ & $\begin{array}{c}11 \mathrm{q} 23 \text { - qter } \\
11 \mathrm{q}\end{array}$ & $\begin{array}{c}(18.2 \%) \\
(42 \%)\end{array}$ & Ghazvini et al., 1996 & & & \\
\hline
\end{tabular}

${ }^{\mathrm{a}}$ The percentage in parentheses is the frequency of occurrence for a given loss.

${ }^{\mathrm{b}}$ See cell line key. 
Table 3 (cont'd). A Comparison of Losses Seen in Ten Uveal Melanoma Cell Lines Compared with Previously Reported Chromosomal Abnormalities in Uveal Melanoma.

\begin{tabular}{|c|c|c|c|c|c|c|c|}
\hline \multicolumn{2}{|c|}{$\begin{array}{l}\text { Losses Observed in the } \\
\text { Current Study }\end{array}$} & \multirow{2}{*}{$\begin{array}{c}\text { Cell Lines }^{\mathbf{b}} \\
1,2,3,4,6,7,8,10\end{array}$} & \multicolumn{2}{|c|}{ Losses Reported by CGH } & Reference & \multirow[t]{2}{*}{$\begin{array}{c}\text { Losses Reported by } \\
\text { Classical Cytogenetics }\end{array}$} & \multirow[t]{2}{*}{ Reference } \\
\hline 12 p13.3 - pter & $(80 \%)$ & & $12 p$ & $(9.1 \%)$ & Speicher et al., 1994 & & \\
\hline $13 q 34$ - qter & $(50 \%)$ & $2,4,6,7,8$ & & & & & \\
\hline $14 q 32.3$ - qter & $(60 \%)$ & $2,3,5,7,8,9$ & & & & & \\
\hline $16 q 24-$ qter & $(50 \%)$ & $2,7,8,9,10$ & $16 \mathrm{q}$ & $(18.2 \%)$ & $"$ & & \\
\hline $\begin{array}{c}18 p 11.31 \text { - pter } \\
18 q 23 \text { - qter }\end{array}$ & $\begin{array}{l}(80 \%) \\
(70 \%)\end{array}$ & $\begin{array}{c}2,3,4,6,7,8,9,10 \\
1,2,4,7,8,9,10\end{array}$ & & & & & \\
\hline $21 \mathrm{q} 22.3$ - qter & $(50 \%)$ & $2,7,8,9,10$ & & & & & \\
\hline
\end{tabular}

${ }^{a}$ The percentage in parentheses is the frequency of occurrence for a given loss.

${ }^{\mathrm{b}}$ See cell line key. 
Table 4. A Correlation of Copy Number Abnormalities in Ten Uveal Melanoma Cell Lines and Genes Associated with Uveal Melanoma or its Predisposing Factors.

\begin{tabular}{|c|c|c|c|c|c|c|}
\hline \multicolumn{2}{|c|}{$\begin{array}{l}\text { Gain/Loss Observed in } \\
\text { Current Study } \\
\end{array}$} & \multirow{2}{*}{$\begin{array}{c}\text { Cell Lines }^{\mathbf{b}} \\
1,2,3,6,7,8 \\
1,3,4,6,7,8\end{array}$} & \multirow{2}{*}{$\begin{array}{c}\text { Gene } \\
\text { TNFSF6 }\end{array}$} & \multirow{2}{*}{$\frac{\text { Locus }^{\mathbf{c}}}{1 \mathrm{q} 23}$} & \multirow{2}{*}{$\begin{array}{r}\text { Function } \\
\text { tumor necrosis factor ligand }\end{array}$} & \multirow[t]{2}{*}{ Source $^{c, d}$} \\
\hline $\begin{array}{l}+1 \mathrm{q} 21.1-1 \mathrm{q} 21.2 \\
+1 \mathrm{q} 21.2\end{array}$ & $\begin{array}{l}(60 \%) \\
(60 \%)\end{array}$ & & & & & \\
\hline$-2 q 31-q 32$ & $(20 \%)$ & 9,10 & $\begin{array}{l}\text { alpha4 integrin } \\
\text { subunit gene, } \\
\text { CAT gene }\end{array}$ & $2 q 31-q 32$ & $\begin{array}{l}\text { transcriptional regulator of alpha4 integrin } \\
\text { subunit gene in metastasis, alpha4 promoter } \\
\text { fused to CAT gene }\end{array}$ & Larouche et al., 1998 \\
\hline$+6 \mathrm{p} 21.2-\mathrm{p} 21.3$ & $(50 \%)$ & $1,2,7,9,10$ & HLA-G & $6 \mathrm{p} 21.3$ & histocompatability antigen & OMIM \\
\hline$-6 q 14-q 21$ & $(50 \%)$ & $1,2,6,7,10$ & $?$ & $6 q 12-q 21$ & putative tumor suppressor gene & Copeman, 1992 \\
\hline$-6 q 21-q 22.2$ & $(60 \%)$ & $1,2,3,6,7,10$ & AIM1 & $6 q 21$ & absent in melanoma & OMIM \\
\hline$+7 q 21.2-q 21.3$ & $(60 \%)$ & $1,2,3,7,8,9$ & MDR1 & $7 q 21.1-q 21.2$ & $\begin{array}{l}\text { produces P-gp pump protein, causes drug } \\
\text { resistance in cancer cells }\end{array}$ & Dunne et al., 1998 \\
\hline $\begin{array}{l}+8 \mathrm{q} 24.1-\mathrm{q} 24.2 \\
+8 \mathrm{q} 24.2-\mathrm{q} 24.3 \\
+8 \mathrm{q} 24.3\end{array}$ & $\begin{array}{l}(60 \%) \\
(60 \%) \\
(50 \%)\end{array}$ & $\begin{array}{c}1,2,4,5,8,10 \\
1,2,5,6,8,10 \\
1,2,5,8,10\end{array}$ & c-myc & $\begin{array}{l}8 \mathrm{q} 24 \\
"\end{array}$ & oncogene & $\begin{array}{l}\text { Blom et al., } 1997 \\
\text { Chana et al.,1999 }\end{array}$ \\
\hline-9 p23 - p24 & $(50 \%)$ & $6,7,8,9,10$ & CDKN2 (p16) & $9 \mathrm{p} 21$ & $\begin{array}{l}\text { tumor suppressor, involved in FAM-M and } \\
\text { Li-Fraumeni }\end{array}$ & Singh et al., 1996a \\
\hline \multirow[t]{4}{*}{-9 p24 - pter } & $(90 \%)$ & $1,2,4,5,6,7,8,9,10$ & $\begin{array}{l}\text { FCM critical } \\
\text { region }\end{array}$ & $9 \mathrm{p} 13-\mathrm{p} 22$ & familial cutaneous melanoma critical region & Houlston and Damato, 1999 \\
\hline & & & & & & $\begin{array}{l}\text { Merbs and Sidransky, } 1999 \\
\text { Singh et al., 1996b }\end{array}$ \\
\hline & & & CMM2 & $9 \mathrm{p} 21$ & $\begin{array}{l}\text { cutaneous malignant melanoma, also } \\
\text { involved in FAM-M }\end{array}$ & OMIM \\
\hline & & & TYRP1 & $9 \mathrm{p} 23$ & tyrosinase-related protein 1 & OMIM \\
\hline
\end{tabular}

${ }^{a}$ The percentage in parentheses is the frequency of occurrence for a given abnormality.

${ }^{b}$ See cell line key.

'Loci without an OMIM reference were pinpointed using the International RH Mapping Consortium - GeneMap '99 at http://www.ncbi.nlm.nih.gov/genemap99. A number of these loci are not identical to those identified in the current study. The International RH Mapping Consortium recognizes the presence of positional discrepancies in the published map. Therefore, we have chosen to list these loci due to their linkage with uveal melanoma and their general proximity to the copy number abnormalities identified in this study.

${ }^{\mathrm{d}}$ All references to OMIM refer to Online Mendelian Inheritance in Man, http://www.ncbi.nlm.nih.gov/Omim/ and were collected by doing a keyword search of

"uveal melanoma" and "melanoma." 
Table 4 (cont'd). A Correlation of Copy Number Abnormalities in Ten Uveal Melanoma Cell Lines and Genes Associated with Uveal Melanoma or its Predisposing Factors.

\begin{tabular}{|c|c|c|c|c|c|c|}
\hline \multicolumn{2}{|c|}{$\begin{array}{c}\text { Gain/Loss Observed in } \\
\text { Current Study } \\
\end{array}$} & \multirow{2}{*}{$\begin{array}{l}\text { Cell Lines }^{\mathbf{b}} \\
2,4,7,8,9,10\end{array}$} & \multirow{2}{*}{$\begin{array}{c}\text { Gene } \\
\text { DMBT1 }\end{array}$} & \multirow{2}{*}{$\begin{array}{c}\text { Locus }^{\mathbf{c}} \\
10 \mathrm{q} 25.3-\mathrm{q} 26.1\end{array}$} & \multirow{2}{*}{$\begin{array}{l}\text { Function } \\
\text { deleted in malignant brain tumors }\end{array}$} & \multirow{2}{*}{$\begin{array}{ll} & \text { Source }^{\text {c,d }} \\
\text { OMIM }\end{array}$} \\
\hline$-10 \mathrm{q} 26.2$ qter & $(60 \%)$ & & & & & \\
\hline$+11 \mathrm{p} 14-\mathrm{p} 15.1$ & $(50 \%)$ & $3,4,5,7,8$ & $\begin{array}{c}\text { HRAS } \\
"\end{array}$ & $11 \mathrm{p} 15.5$ & $\begin{array}{l}\text { v-Ha-Ras oncogene } \\
\text { " }\end{array}$ & $\begin{array}{l}\text { Singh et al., 1996a } \\
\text { OMIM }\end{array}$ \\
\hline$-11 \mathrm{q} 25$ - qter & $(80 \%)$ & $1,2,5,6,7,8,9,10$ & tyrosinase & $11 q 13.5-q 14.1$ & melanoma associated antigen & Luyten et al., 1998 \\
\hline-12 p13.3 - pter & $(80 \%)$ & $1,2,3,4,6,7,8,10$ & $\begin{array}{l}\text { KRAS } \\
"\end{array}$ & $12 \mathrm{p} 12.1$ & $\begin{array}{l}\mathrm{v}-\mathrm{Ki} \text {-Ras } 2 \text { oncogene } \\
\text { " }\end{array}$ & $\begin{array}{l}\text { Singh et al., 1996a } \\
\text { OMIM }\end{array}$ \\
\hline$-13 q 34-$ qter & $(50 \%)$ & $2,4,6,7,8$ & BRCA2 & $13 q 12.2-q 12.3$ & breast cancer susceptibility locus & Houlston and Damato, 1999 \\
\hline$-16 \mathrm{q} 24-$ qter & $(50 \%)$ & $2,7,8,9,10$ & MC1R & $16 \mathrm{q} 24.3$ & melanocortin 1 receptor & OMIM \\
\hline-17 p13 - pter & $(30 \%)$ & $2,9,10$ & p53 & $17 \mathrm{p} 13.1$ & $\begin{array}{l}\text { tumor suppressor, involved in } \\
\text { Li-Fraumeni }\end{array}$ & Singh et al., 1996a \\
\hline$+17 q 11.1-q 11.2$ & $(50 \%)$ & $3,4,5,6,8$ & NF1 & $17 \mathrm{q} 11.2$ & neurofibro matosis & OMIM \\
\hline$+17 \mathrm{q} 21.3-\mathrm{q} 22$ & $(50 \%)$ & $1,5,6,7,8$ & BRCA1 & $17 q 21$ & breast cancer & OMIM \\
\hline$+17 \mathrm{q} 22-\mathrm{q} 24$ & $(80 \%)$ & $1,2,5,6,7,8,9,10$ & PRKCA & $17 \mathrm{q} 22-\mathrm{q} 23.2$ & protein kinase $\mathrm{C}$ & OMIM \\
\hline$+19 \mathrm{p} 13.3-\mathrm{q} 12$ & $(50 \%)$ & $1,3,6,7,10$ & STK11 & $19 \mathrm{p} 13.3$ & serine/threonine protein kinase II & OMIM \\
\hline$+19 \mathrm{q} 12-\mathrm{q} 13.1$ & $(60 \%)$ & $1,3,6,7,8,10$ & MEL & $19 \mathrm{p} 13.2$ - cen & oncogene MEL, Ras-associated protein & OMIM \\
\hline$+19 q 13.1-q 13.2$ & $(50 \%)$ & $3,6,7,8,10$ & MIA & $\begin{array}{l}19 q 13.32- \\
q 13.33\end{array}$ & melanoma inhibitory activity & OMIM \\
\hline$+22 q 11.2-q 12.1$ & $(90 \%)$ & $1,2,4,5,6,7,8,9,10$ & $\begin{array}{l}\text { EWSR 1 } \\
\text { LFS } \\
\text { NF2 }\end{array}$ & $\begin{array}{l}22 q 12 \\
22 q 12.1 \\
22 q 12.2\end{array}$ & $\begin{array}{l}\text { Ewing sarcoma breakpoint } \\
\text { Li-Fraumeni } \\
\text { neurofibromatosis }\end{array}$ & $\begin{array}{l}\text { OMIM } \\
\text { OMIM } \\
\text { OMIM }\end{array}$ \\
\hline
\end{tabular}

${ }^{\mathrm{a}}$ The percentage in parentheses is the frequency of occurrence for a given abnormality.

${ }^{\mathrm{b}}$ See cell line key.

'Loci without an OMIM reference were pinpointed using the International RH Mapping Consortium - GeneMap '99 at

http://www.ncbi.nlm.nih.gov/genemap99. A number of these loci are not identical to those identified in the current study. The International RH Mapping

Consortium recognizes the presence of positional discrepancies in the published map. Therefore, we have chosen to list these loci due to their linkage with uveal melanoma and their general proximity to the copy number abnormalities identified in this study.

${ }^{\mathrm{d}}$ All references to OMIM refer to Online Mendelian Inheritance in Man, http://www.ncbi.nlm.nih.gov/Omim/ and were collected by doing a keyword search of "uveal melanoma" and "melanoma." 
3 as a whole. Gains were also seen on $6 \mathrm{p}$ and distal 8q, while consistent losses were found on 6q (Fig. 16). A thorough evaluation of chromosomes 3, 6, and 8 was critical because they are frequently reported as aberrant in uveal melanoma. The cell line MEL285 approached monosomy 3, as a prominent loss extended from the p-terminus to $3 q 21$, but the remainder of the chromosome traced the normal midline of the CGH profile. Loss of distal 3p was seen in the quantitative comparison data (QCD) (Fig. 19) for MEL290, OCM3, OCM8, and OM431. Several cell lines exhibited tendencies toward loss of portions of chromosome 3, including 92-1, MEL290, OCM-3, OCM-8, and OM431. These tendencies did not exceed the threshold for loss. The trend seen in 92-1 was toward a loss of $3 q$, whilst the others were for loss of $3 p$. The $p$-arm of chromosome 6 was gained in 92-1, MEL270, OMM-1, and OMM-2.3, while 6q was lost in 92-1, MEL270, MEL285, OCM-3, OCM-8, OMM-1, and OMM-2.3. Three cell lines showed loss on both 6q and distal 3p (MEL285, OCM-3, and OM431). A focal gain at 8q24 was also seen in 5 of the 10 cell lines (92-1, MEL290, OCM-1, OCM-3, and OM431). The entire chromosome 8 was gained in MEL270, while only 8q was gained in OMM-2.3.

\section{Spectral Karyotyping.}

SKY identified numerical and structural aberrations on a per-cell basis in each of the cell lines. For our SKY analysis, a total of 2,334 chromosome structures were analyzed, with the percent of aberrations ranging from $0 \%$ for the $\mathrm{Y}$ chromosome to 62.96\% for chromosome 1 (Table 5). Chromosomes 1, 3, 8 and $\mathrm{X}$ contained aberrations in all of the cell lines. Chromosomes 1, 3, 7, 12, 17 and 20 were aberrant more than 50\% of the time collectively across all cell lines. Frequent structural and numerical aberrations were tabulated as those that occurred across 3 or more cell lines (Table 5). 
The high frequency of these aberrations suggests that chromosomes 1, 3, 7, 12, 17 and 20 may contain genes of significance in the establishment and/or progression of uveal malignant melanoma. Chromosomes 1, 3, 8, 17, 19, 20, and 22 were often involved in numerical aberrations (Table 5). Complex aberrations involving more than 2 chromosomes were seen frequently in MEL290, OCM-1, OCM-3, and OCM-8 (Fig. 21).

Partially deleted, or truncated, copies of chromosome 3 were seen in OCM-3, OCM-8, and OM431. There were multiple copies of marker chromosomes in OCM-1 and OM431 that SKY classified as chromosome 3 material. Additional copies of $6 \mathrm{p}$ were found in MEL270 and OCM-8, and additional copies of 8q were found in OCM-3 and OCM- 8 . There were no $(3 ; 6)$ or $(3 ; 8)$ rearrangements in any of the cell lines.

Five chromosomal aberrations were observed across multiple cell lines (Fig. 22). The most prevalent abnormalities were $+\operatorname{der}(1) \mathrm{t}(1 ; 10)(\mathrm{q} 10 ; \mathrm{q} 10)(92-1$, OCM-8, OM431, OCM-3, and MEL285) and $+\operatorname{der}(2) t(2 ; 12)(\mathrm{p} 10 ; \mathrm{q} 10)$ 92-1, OCM-8, OM431, OCM-3, and OCM-1), each evidenced in 5 cell lines. Other interactions, including $+\operatorname{der}(20) \mathrm{t}(\mathrm{X} ; 20)(? ; \mathrm{q}$ ?) (OCM-8, OCM-3, OCM-1, and OMM-2.3) and the persistent reciprocal t(3;10)(p25;q25) (OCM-8, OM431, OMM-2.3, and MEL270), were each seen in four cell lines. Three distinct rearrangements were seen between chromosomes 3 and 7, but poor banding quality prevented clarification beyond $+\operatorname{der}(3) t(3 ; 7)(? ; ?)$ or $+\operatorname{der}(7) t(3 ; 7)(? ; ?)$ for these non-reciprocal translocations. These rearrangements between chromosomes 3 and 7 were seen in OCM-8, OM431, OCM-1, and OMM-1. Note that the cell line OCM-8 was the only line evidencing some form of all five of these interactions. 
Table 5. Summary of Aberrations Identified by SKY in Ten Uveal Melanoma Cell

\section{Lines}

\begin{tabular}{|c|c|c|c|c|c|}
\hline $\begin{array}{c}\text { Chromoso } \\
\text { me }\end{array}$ & $\begin{array}{c}\text { Range Percent } \\
\text { Aberrations }^{\mathrm{a}}\end{array}$ & $\begin{array}{c}\text { Mean Percent } \\
\text { Aberrations }\end{array}$ & $\begin{array}{c}\text { Lines with } \\
\text { Aberrations }\end{array}$ & Structural Aberrations ${ }^{\mathbf{b}}$ & Frequent Aberrations ${ }^{b, c, d}$ \\
\hline 1 & $25-100$ & 62.96 & 10 & $\mathrm{t}$, dic, del, ins, mr & $\begin{array}{c}\operatorname{der}(1 ; 10)[5] \\
\mathrm{t}(1 ; 2)[3] \\
\operatorname{der}(1 ; 7)[3] \\
\operatorname{der}(1 ; 16)[3]\end{array}$ \\
\hline 2 & $0-100$ & 49.29 & 7 & $\mathrm{t}$, dic, del, ins, $\mathrm{mr}$ & $\operatorname{der}(2 ; 12)[5]$ \\
\hline 3 & $16.67-66.67$ & 51.43 & 10 & $\mathrm{t}$, dic, del, ins, mr & $\begin{array}{c}\operatorname{der}(3 ; 7) / t(3 ; 7)[5] \\
t(3 ; 10)[4]\end{array}$ \\
\hline 4 & $0-52.63$ & 22.73 & 7 & $\mathrm{t}, \mathrm{del}$ & \\
\hline 5 & $0-88.89$ & 42.54 & 7 & $\mathrm{t}, \mathrm{del}, \mathrm{i}$ & $\operatorname{dup}(5 p)[3]$ \\
\hline 6 & $0-66.67$ & 37.84 & 8 & $\mathrm{t}, \mathrm{del}, \mathrm{i}$ & \\
\hline 7 & $0-90.63$ & 59.90 & 9 & $\mathrm{t}$, dic, del, mr & $\mathrm{t}(7 ; 17)[3]$ \\
\hline 8 & $8.33-75$ & 47.17 & 10 & $\mathrm{t}, \mathrm{del}, \mathrm{i}, \mathrm{mr}$ & $\operatorname{der}(8 ; 11)[3]$ \\
\hline 9 & $0-66.67$ & 25.24 & 6 & $\mathrm{t}$ & \\
\hline 10 & $0-80$ & 44.74 & 8 & $\mathrm{t}$, ins, del & \\
\hline 11 & $0-75$ & 43.65 & 7 & $\mathrm{t}$, ins, del & \\
\hline 12 & $0-85.71$ & 61.81 & 8 & $\mathrm{t}$, ins, del & $\begin{array}{l}\operatorname{dup}(12 p)[3] \\
\operatorname{dup}(12 q)[3]\end{array}$ \\
\hline 13 & $0-50$ & 22.99 & 7 & $\mathrm{t}$ & \\
\hline 14 & $0-50$ & 12.64 & 4 & $\mathrm{t}, \mathrm{mr}, \mathrm{R}$ & \\
\hline 15 & $0-66.67$ & 27.45 & 4 & $\mathrm{t}, \mathrm{mr}$ & \\
\hline 16 & $0-72.73$ & 49.59 & 8 & $\mathrm{t}, \mathrm{mr}$ & $\operatorname{der}(16 ; 17)[3]$ \\
\hline 17 & $0-100$ & 57.81 & 8 & $\mathrm{t}, \mathrm{mr}$ & \\
\hline 18 & $0-46.67$ & 14.94 & 3 & $\mathrm{t}, \mathrm{mr}$ & \\
\hline 19 & $0-75$ & 30.36 & 7 & $\mathrm{t}, \mathrm{mr}$ & \\
\hline 20 & $0-100$ & 57.45 & 6 & $\mathrm{t}, \mathrm{mr}$ & $\operatorname{der}(20 ; X)[4]$ \\
\hline 21 & $0-100$ & 15.15 & 8 & $\mathrm{t}$ & \\
\hline 22 & $0-57.89$ & 18.10 & 5 & $\mathrm{t}, \mathrm{i}$ & \\
\hline $\mathrm{X}$ & $11.11-100$ & 46.51 & 10 & $\mathrm{t}, \mathrm{del}$ & \\
\hline $\mathrm{Y}$ & $0-\mathrm{ND}^{\mathrm{e}}$ & 0 & 0 & & \\
\hline \multicolumn{6}{|c|}{$\begin{array}{l}\text { a The range of percent aberrations is determined by the number of aberrant copies of a given chromosome in all SKY spreads for a } \\
\text { cell line compared to the number of normal copies of that same chromosome in that same cell line } \\
\text { b del = deletion, der = derivative, dic = dicentric, dup = duplication, } \mathrm{i}=\text { isochromosome, ins = insertion, mr = multiple rearrangement, } \\
\mathrm{R}=\text { Robertsonian translocation, } \mathrm{t}=\text { translocation. All nomenclature, except "mr," according to ISCN } 1995 \text {. } \\
\text { c The number in brackets is the number of cell lines in which the particular aberration was identified. } \\
\text { d Redundancies in the data set have been eliminated. } \\
\text { e } \\
\text { The notation "ND" indicates that there were no observations, normal or aberrant, for that chromosome. There was no data in 92-1, } \\
\text { MEL270, MEL285, MEL290, OCM-1, OCM-3, OM431, and OMM1 for chromosome Y. }\end{array}$} \\
\hline
\end{tabular}


A.

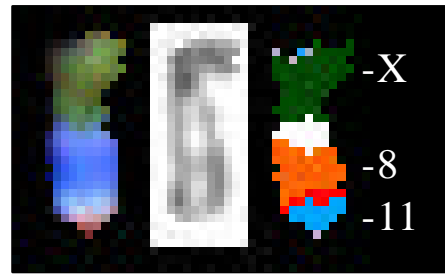

B.

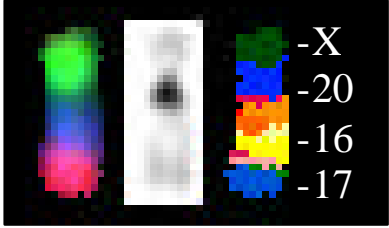

C.
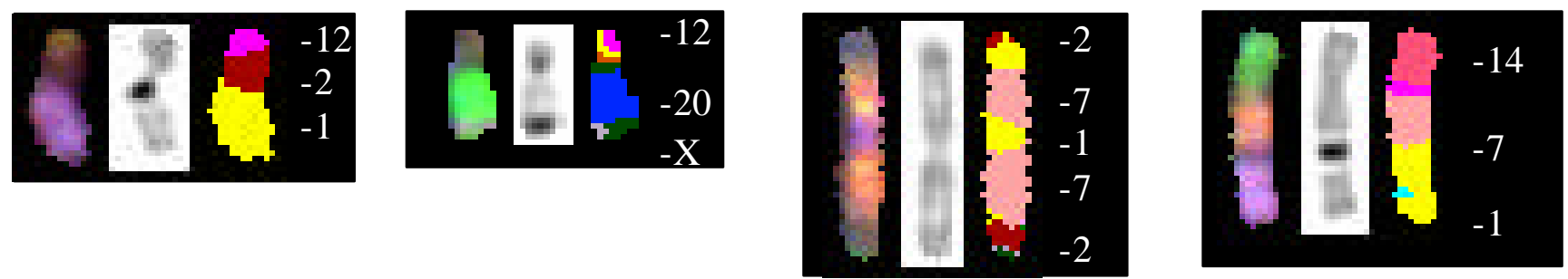

D.
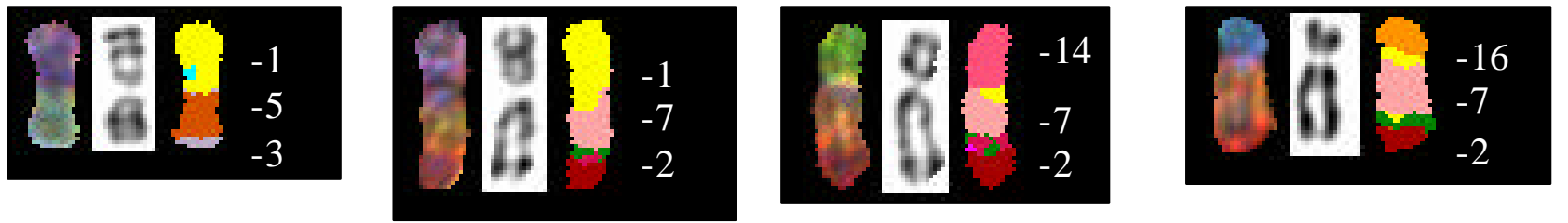

Figure 21. Complex Aberrations Detected by Spectral Karyotyping. SKY employs 24 distinctly labeled chromosome-specific probes, thus allowing for the identification of all the chromosomes involved in complex rearrangements. SKY was performed on 10 uveal melanoma cell lines and revealed complex chromosomal rearrangements in 4 of the lines: (A) MEL290, (B) OCM-1, (C) OCM-3 and (D) OCM-8. 
A.

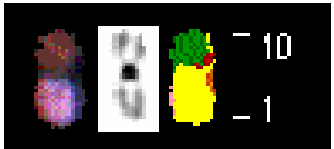

$+\operatorname{der}(1) \mathrm{t}(1 ; 10)(\mathrm{q} 10 ; \mathrm{q} 10)$

B.

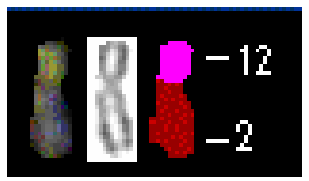

\author{
$+\operatorname{der}(2) \mathrm{t}(2 ; 12)(\mathrm{p} 10 ; \mathrm{q} 10)$
}

C.

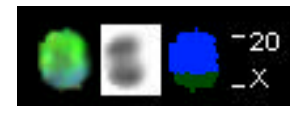

$+\operatorname{der}(20) t(X ; 20)(? ; q ?)$

D.
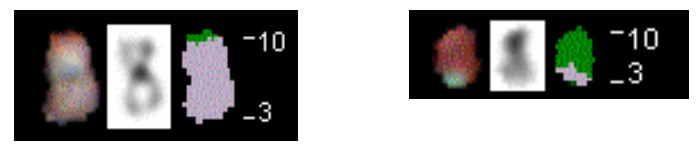

$\mathrm{t}(3 ; 10)(\mathrm{p} 25 ; \mathrm{q} 25)$

E.
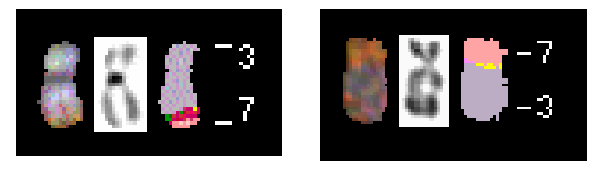

$+\operatorname{der}(3) \mathrm{t}(3 ; 7)(? ; ?)$

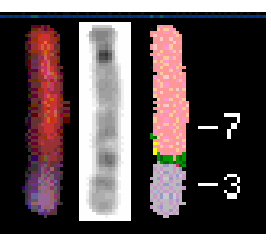

$+\operatorname{der}(7) \mathrm{t}(3 ; 7)(? ; ?)$

Figure 22. Chromosome Rearrangements Seen Across Ten Uveal Melanoma Cell Lines. (A) A $+\operatorname{der}(1) \mathrm{t}(1 ; 10)(\mathrm{q} 10 ; \mathrm{q} 10)$ was seen in $92-1$, OCM-3, OCM-8, OM431 and MEL285. (B) A $+\operatorname{der}(2) \mathrm{t}(2 ; 12)(\mathrm{p} 10 ; \mathrm{q} 10)$ was seen in $92-1$, OCM-1, OCM-3, OCM-8 and OM431. Note that these interactions were observed in 5 cell lines each. (C) The cell lines OCM-1, OCM-3, OCM-8 and OMM2.3 all evidenced a $+\operatorname{der}(20) t(X ; 20)(?, q$ ?). (D) Four cell lines (MEL270, OCM-8, OM431 and OMM2.3) exhibited the reciprocal t(3;10)(p25;q25). (E) Additional interactions involving chromosome 3 , all between dromosomes 3 and 7 , were seen as 2 distinct $+\operatorname{der}(3) \mathrm{t}(3 ; 7)(?, ?)$ and $\mathrm{a}+\operatorname{der}(7) \mathrm{t}(3 ; 7)(? ; ?)$ that were not elucidated by banding analyses. These interactions all occurred in OCM-1, OCM-8, OM431 and OMM1. Note that the only cell line containing some form of all five of these interactions was OCM-8. Interactions observed in multiple cell lines, such as these, are likely linked to the development of their associated cell line, and warrant further study. These interactions are also likely associated with copy number abnormalities in the genome, which lead to enhanced or diminished gene products during development. 


\section{Interphase Fluorescence in situ Hybridization.}

Locus-specific FISH probes for 3p, 3q, and 3cen allowed further study of chromosome 3 in the cell lines (Table 6). Two hundred nuclei were scored at random for each probe and each cell line. In the diploid control, $86 \%$ of the normal male nuclei analyzed had 2 signals for 3 cen. Our cell lines were heterogeneous in chromosome 3 complement according to centromeric-FISH, showing 0 to 6 copies of 3 cen (Table 6), and averaging 0.99 to 1.53 copies per cell. The percentage of 3 cen loss ( 0 or 1 copies present) was highest in MEL285 (72.5\%) and lowest in OCM-1 (49.5\%). The dominant population in each cell line had one 3cen hybridization signal per nucleus (ranging from $34 \%$ of cells for $92-1$ to $45.5 \%$ of cells for MEL285). Meanwhile, gain of signal $\gtrless 2$ copies) ranged from $2.5 \%$ (OMM-1) to $19 \%$ (OCM-3) by centromeric-FISH.

Results for the telomere probes of the diploid control showed that $93 \%$ of cells had 2 signals for both $3 p$ and $3 q$. However, we saw a broad range of signals for $3 p$ (0 to 10 copies) and 3q (0 to 12) in individual cells across all cell lines (Table 6). In contrast to the FISH findings for $3 c e n$, no more than $16 \%$ of nuclei had one $3 p$ signal and no more than 4\% had one 3q signal (both seen in MEL290). Four cell lines had at least two 3p and/or two 3q signals in every cell scored (Table 6).

\section{Comparison of Techniques.}

A quantitative analysis was used to determine the relationship between $\mathrm{CGH}$, SKY and FISH. Measurements of relative balance were generated from pixel quantification in CGH and SKY to show the association between the 2 techniques for all chromosomes (Fig. 23). Regression analysis of this data revealed significant association 
Table 6. Chromosome 3 FISH Signals for Ten Uveal Melanoma Cell Lines

\begin{tabular}{|c|c|c|c|c|c|c|c|}
\hline Cell Line & 3 cen \pm SE & 3 cen Range ${ }^{\mathrm{a}}$ & $3 p \pm S E$ & $3 p$ Range $^{a}$ & $3 q \pm S E$ & $3 q$ Range $^{a}$ & FISH RCE $^{\mathrm{b}} \pm \mathrm{SE}$ \\
\hline Control & $2.04 \pm 0.03$ & $0-4$ & $1.99 \pm 0.02$ & $1-3$ & $1.96 \pm 0.02$ & $0-3$ & $1.99 \pm 0.01$ \\
\hline $92-1$ & $1.23 \pm 0.07$ & $0-4$ & $4.14 \pm 0.05$ & $2-6$ & $3.76 \pm 0.04$ & $2-5$ & $3.05 \pm 0.06$ \\
\hline MEL270 & $1.12 \pm 0.07$ & $0-3$ & $3.10 \pm 0.07$ & $0-6$ & $3.21 \pm 0.06$ & $0-7$ & $2.48 \pm 0.05$ \\
\hline MEL285 & $1.06 \pm 0.06$ & $0-3$ & $2.41 \pm 0.06$ & $0-6$ & $3.08 \pm 0.05$ & $0-8$ & $2.18 \pm 0.05$ \\
\hline MEL290 & $1.53 \pm 0.07$ & $0-4$ & $2.03 \pm 0.06$ & $0-6$ & $2.26 \pm 0.04$ & $1-5$ & $1.94 \pm 0.04$ \\
\hline OCM-1 & $1.50 \pm 0.06$ & $0-4$ & $4.90 \pm 0.07$ & $1-10$ & $5.45 \pm 0.07$ & $3-11$ & $3.95 \pm 0.08$ \\
\hline OCM-3 & $1.50 \pm 0.08$ & $0-5$ & $2.96 \pm 0.06$ & $0-7$ & $4.37 \pm 0.08$ & $0-9$ & $2.94 \pm 0.06$ \\
\hline OCM-8 & $1.21 \pm 0.07$ & $0-6$ & $3.29 \pm 0.06$ & $2-7$ & $4.64 \pm 0.09$ & $2-12$ & $3.05 \pm 0.07$ \\
\hline OM431 & $1.31 \pm 0.07$ & $0-6$ & $3.98 \pm 0.04$ & $2-6$ & $5.51 \pm 0.07$ & $0-11$ & $3.60 \pm 0.08$ \\
\hline OMM1 & $0.99 \pm 0.06$ & $0-3$ & $3.03 \pm 0.04$ & $1-6$ & $3.04 \pm 0.05$ & $0-7$ & $2.35 \pm 0.05$ \\
\hline OMM2.3 & $1.13 \pm 0.06$ & $0-5$ & $4.34 \pm 0.07$ & $2-9$ & $4.22 \pm 0.07$ & $2-9$ & $3.23 \pm 0.07$ \\
\hline
\end{tabular}


between CGH RB and SKY RB for all 22 autosomes across the 9 analyzable cell lines (R $\left.=0.6146, \mathrm{p}=6.513 \times 10^{-21}\right)$. Correlation of CGH and SKY was also seen visually, wherein gain and loss in the CGH profile mirrored specific numerical and structural aberrations in SKY (Fig. 24, note chromosomes 3, 6 and 8).

Of particular interest, were the CGH and SKY correlations in chromosomes 3, 6 and 8, as well as SKY and FISH correlations for chromosome 3 (Fig. 25 and Table 7). Correlation of SKY RCE and FISH RCE for chromosome 3 demonstrated good agreement between the two measurements of copy number $(\mathrm{R}=0.7370, \mathrm{p}=0.0235)($ Fig. 25A), and further correlation of FISH and SKY was also evident visually in the cell line

OCM-1 (Fig. 26). The CGH RB:SKY RB comparison for chromosome 3 also showed significant correlation $(\mathrm{R}=0.6775, \mathrm{p}=0.0449)($ Fig. 25B). Correlations for chromosome $6(\mathrm{R}=0.6825, \mathrm{p}=0.0428)$ and chromosome $8(\mathrm{R}=0.8242, \mathrm{p}=0.0063)$ were both significant (Figs. 25C and D).

\section{TUMOR STUDY.}

\section{Comparative Genomic Hybridization.}

Eighty-two of the 100 cases (46 male, 36 female) were successfully extracted, labeled via DOP-PCR, hybridized, and analyzed. Probe constructs from the remaining 18 DNA isolates were unsuccessful in yielding results, due to a failure to either amplify or hybridize appropriately.

Results for all 82 tumors were evaluated by comparison of the mean ratio profiles; numerous consistencies were found throughout the data and used to derive the minimal common regions as a means of simplifying the data set (Figs. 27, 28 and 29). These 


\section{Uveal Melanoma Cell Line Relative Balance Comparison}

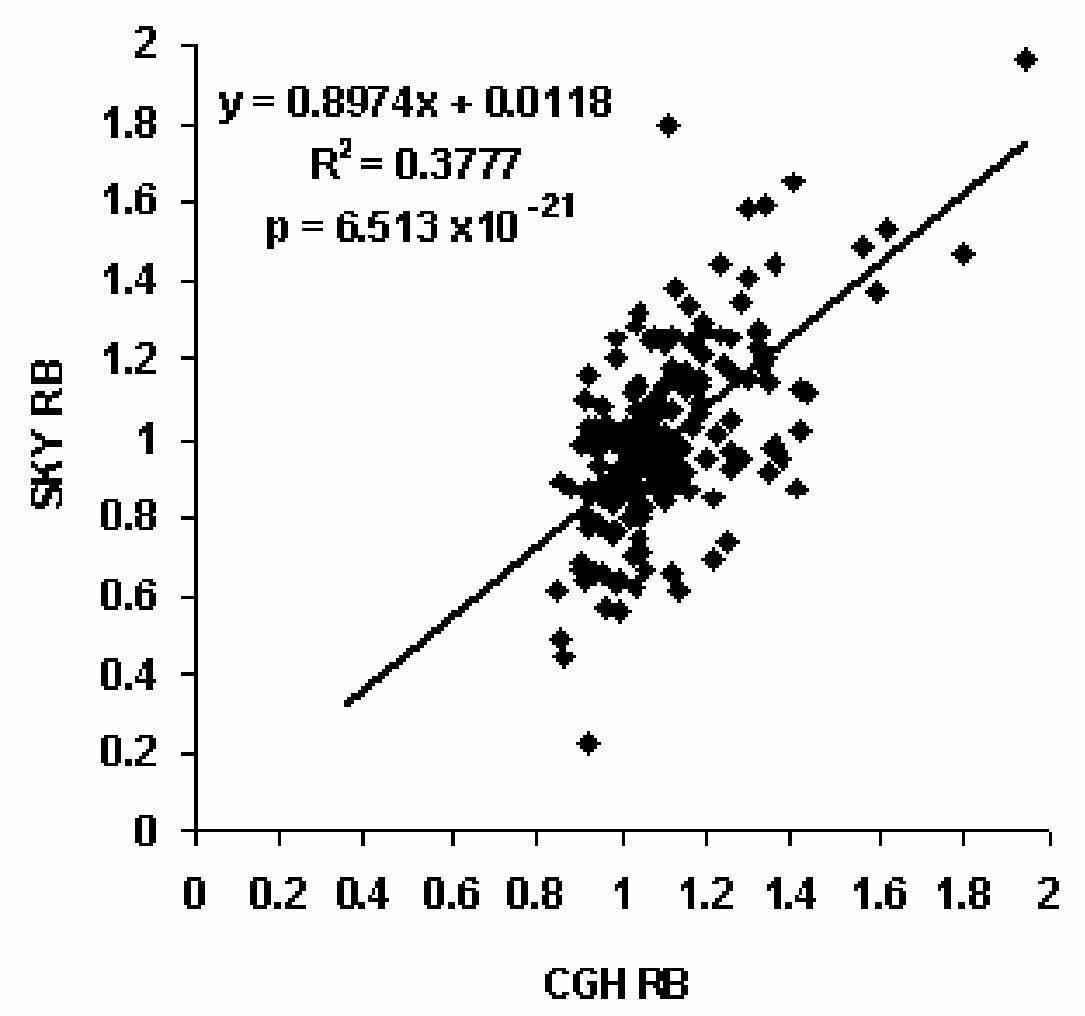

Figure 23. Overall Comparison of Calculated CGH RB and SKY RB Values for the 22 Autosomes in Nine Cell Lines. OCM-3 was excluded fro $m$ this analysis due to inadequate SKY data. The equation for the regression line is provided in the upper left hand corner with the $\mathrm{R}^{2}$-value and $\mathrm{p}$-value immediately below. The y-axis reflects the transformation of internally normalized SKY data into SKY RB values, which converts them from an absolute measure of copy number to a more relative measure comparable to CGH. Transformed CGH data (CGH RB) is plotted on the $\mathrm{x}$ axis. Deviation from the regression line is partially explainable by the inherent difference between the two techniques, as CGH is a genomic average from a pooling of cells and SKY analysis is performed on per-cell basis with results taken from a few metaphase spreads. 

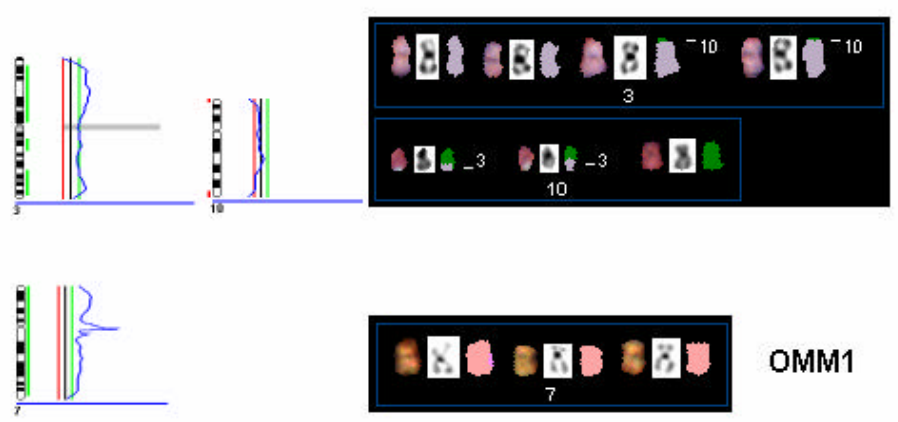

OMM1

B. Structural Chromosome Aberrations

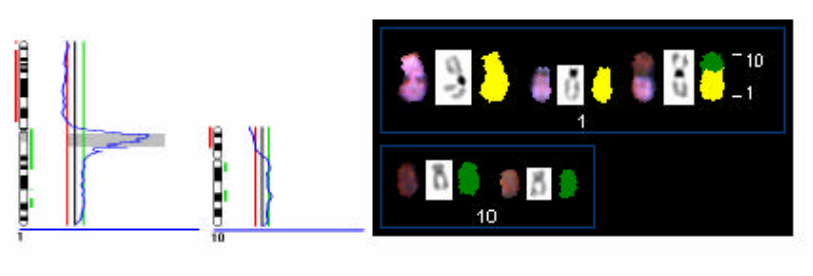

MEL285
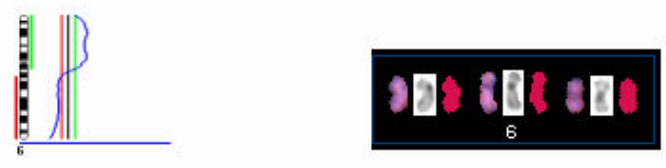

MEL270
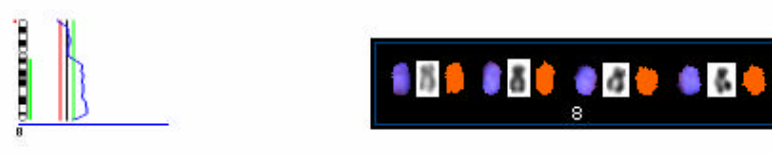

OMM2.3

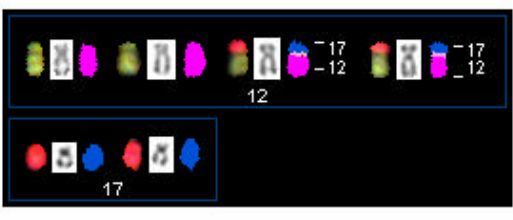

92-1

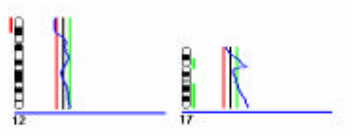

Figure 24. Visual Comparison of CGH and SKY. (A) These examples show how the numerical aberrations in SKY (right) correspond to whole chromosome amplification in CGH (left). Balanced structural aberrations such as the $\mathrm{t}(3 ; 10)$ in OMM2.3 essentially result in a numerical aberration leading to additional complete copies of chromosome 3 . Addition of an entire chromosome, as seen in chromosome 7 from OMM1 (a pseudodiploid cell line), also result in amplification along the length of the $\mathrm{CGH}$ ratio profile. (B) These examples demonstrate correlation between $\mathrm{CGH}$ mean ratio profiles and unbalanced structural aberrations in SKY. The presence of a normal chromosome 1, a 1q, and a der(1;10q) result in gain of $1 \mathrm{q}$ and loss of $1 \mathrm{p}$ in CGH of MEL285. The der(1;10q) adds additional 10q material mirrored by the relative loss of $10 \mathrm{p}$ and gain of $10 \mathrm{q}$ in CGH. In the next example the gain of $6 \mathrm{p}$ and loss of $6 \mathrm{q}$ in $\mathrm{CGH}$ are mirrored by the 2 normal chromosomes 6 (left) and an iso(6p) (right) in SKY. Two normal chromosomes 8 (first 2) accompanied by 2 copies of $8 \mathrm{q}$ (second 2) as seen in SKY, result in a relative deficit of $8 \mathrm{p}$ and a gain of $8 \mathrm{q}$ by CGH in OMM2.3. In the final example, SKY reveals 2 normal copies each of chromosomes 12 and 17 , and 2 copies of a der(12q;17q) that explain the amplification of $17 q$ and loss of $12 p$ seen in the CGH profile of cell line $92-1$. 
A.

Chromosome 3 FISH and SKY Relative Equivalent Comparison

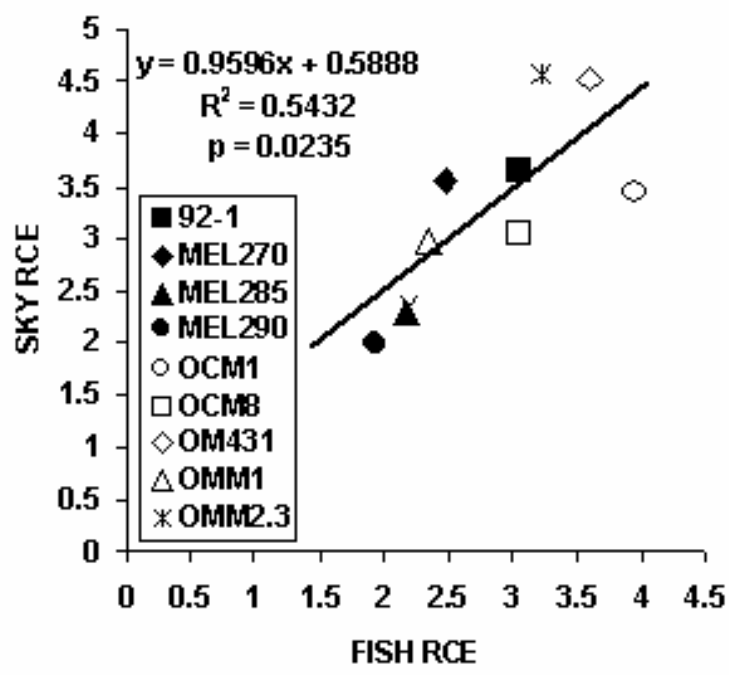

C.

Chromosome 6 CGH and SKY

Relative Balance Comparison

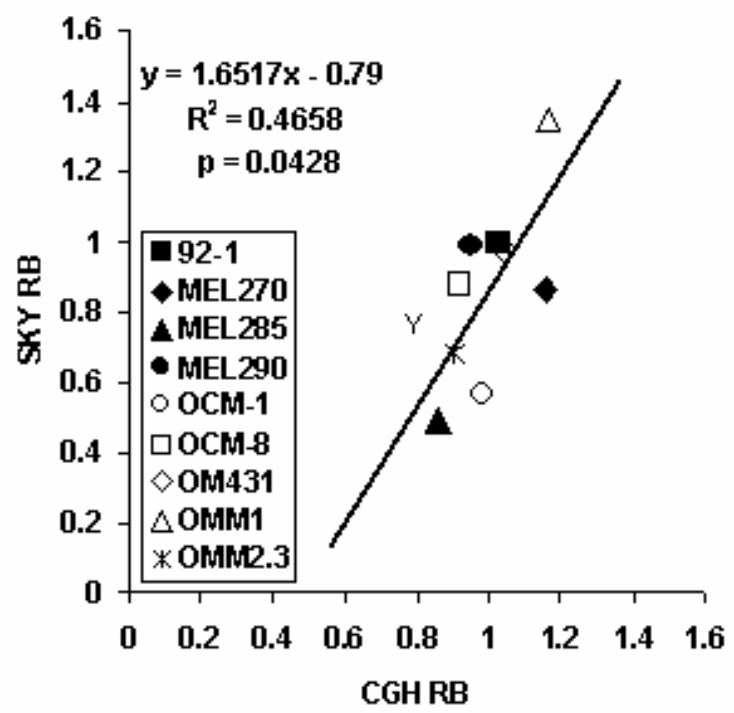

B. Chromosome 3 CGH and SKY

Relative Balance Comparison

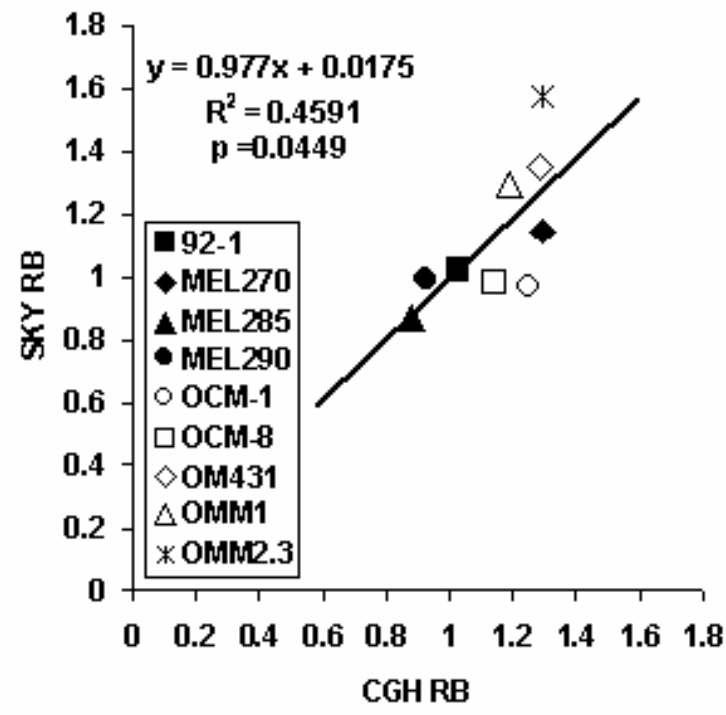

D. Chromosome 8 CGH and SKY Relative Balance Comparison

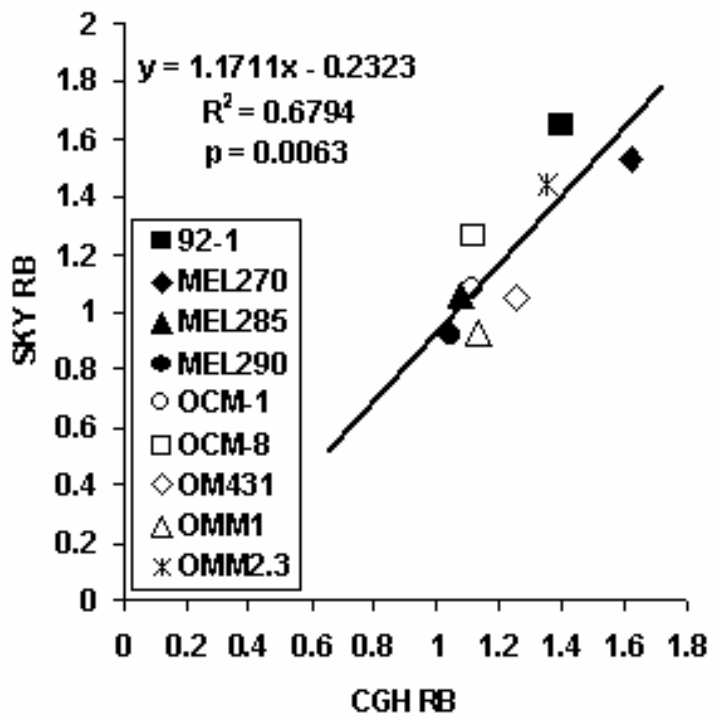

Figure 25. Comparisons of FISH, SKY and CGH Results for Chromosomes 3, 6, and 8, the Three Chromosomes Most Frequently Implicated in Uveal Melanoma. These plots, with trendlines, resulted from the analysis of 9 cell lines, as the cell line OCM-3 was not available for analysis due to a hardware failure during archiving of the data. The regression equations, $\mathrm{R}^{2}$-values, and $\mathrm{p}$-values for each correlation are provided in the upper left-hand corer of each plot. (A) This plot depicts the correlation of SKY RCE and FISH RCE for chromosome 3. Copy number values are read directly from the axes for each technique. (B) This plot represents the correlation calculated between SKY RB and CGH RB values for chromosome 3. Similar correlations between SKY RB and CGH RB are shown for chromosomes 6 (C) and 8 (D). 
Table 7. Comparison of CGH, SKY and FISH in Relative Balance (RB) and Relative Chromosome Equivalents (RCE) for Chromosomes 3, 6 and 8

\begin{tabular}{|c|c|c|c|c|c|c|c|c|}
\hline \multirow[b]{2}{*}{ Cell Line } & \multicolumn{4}{|c|}{ Chromosome 3} & \multicolumn{2}{|c|}{ Chromosome 6} & \multicolumn{2}{|c|}{ Chromosome 8} \\
\hline & CGH RB & SKY RB & SKY RCE & FISH RCE & CGH RB & SKY RB & CGH RB & SKY RB \\
\hline 92-1 & $1.03 \pm 0.06$ & $1.02 \pm 0.11$ & $3.66 \pm 0.58$ & $3.05 \pm 0.06$ & $1.03 \pm 0.03$ & $1.00 \pm 0.05$ & $1.40 \pm 0.06$ & $1.65 \pm 0.08$ \\
\hline MEL 270 & $1.29 \pm 0.05$ & $1.15 \pm 0.03$ & $3.57 \pm 0.14$ & $2.48 \pm 0.05$ & $1.16 \pm 0.03$ & $0.86 \pm 0.09$ & $1.62 \pm 0.05$ & $1.53 \pm 0.06$ \\
\hline MEL285 & $0.88 \pm 0.04$ & $0.87 \pm 0.10$ & $2.30 \pm 0.25$ & $2.18 \pm 0.05$ & $0.86 \pm 0.03$ & $0.49 \pm 0.08$ & $1.08 \pm 0.03$ & $1.06 \pm 0.10$ \\
\hline MEL290 & $0.93 \pm 0.03$ & $0.99 \pm 0.06$ & $1.99 \pm 0.12$ & $1.94 \pm 0.04$ & $0.95 \pm 0.02$ & $0.99 \pm 0.05$ & $1.05 \pm 0.02$ & $0.92 \pm 0.03$ \\
\hline OCM-1 & $1.25 \pm 0.03$ & $0.97 \pm 0.04$ & $3.45 \pm 0.13$ & $3.95 \pm 0.08$ & $0.99 \pm 0.02$ & $0.56 \pm 0.01$ & $1.12 \pm 0.02$ & $1.08 \pm 0.02$ \\
\hline OCM-3 & $1.20 \pm 0.04$ & $\mathrm{ND}^{\mathrm{a}}$ & $\mathrm{ND}^{\mathrm{a}}$ & $2.94 \pm 0.06$ & $0.96 \pm 0.02$ & $\mathrm{ND}^{\mathrm{a}}$ & $1.17 \pm 0.06$ & $\mathrm{ND}^{\mathrm{a}}$ \\
\hline OCM-8 & $1.14 \pm 0.04$ & $0.98 \pm 0.06$ & $3.05 \pm 0.15$ & $3.05 \pm 0.07$ & $0.92 \pm 0.02$ & $0.87 \pm 0.05$ & $1.12 \pm 0.05$ & $1.27 \pm 0.03$ \\
\hline OM431 & $1.28 \pm 0.05$ & $1.35 \pm 0.07$ & $4.52 \pm 0.26$ & $3.60 \pm 0.08$ & $1.04 \pm 0.02$ & $0.98 \pm 0.09$ & $1.25 \pm 0.05$ & $1.05 \pm 0.11$ \\
\hline OMM1 & $1.19 \pm 0.08$ & $1.30 \pm 0.03$ & $2.97 \pm 0.06$ & $2.35 \pm 0.05$ & $1.16 \pm 0.07$ & $0.67 \pm \mathrm{ND}^{\mathrm{a}}$ & $1.13 \pm 0.04$ & $0.93 \pm 0.15$ \\
\hline OMM2.3 & $1.29 \pm 0.05$ & $1.58 \pm 0.06$ & $4.56 \pm 0.17$ & $3.23 \pm 0.07$ & $0.91 \pm 0.02$ & $0.68 \pm 0.08$ & $1.35 \pm 0.06$ & $1.44 \pm 0.15$ \\
\hline
\end{tabular}

"ND indicates "No Data." OCM-3 was the cell line eliminated from the SKY analysis. This value is also given as the standard error for chromosome 6 in OMM1, because there was only one spread bearing a chromosome 6 for that cell line. 

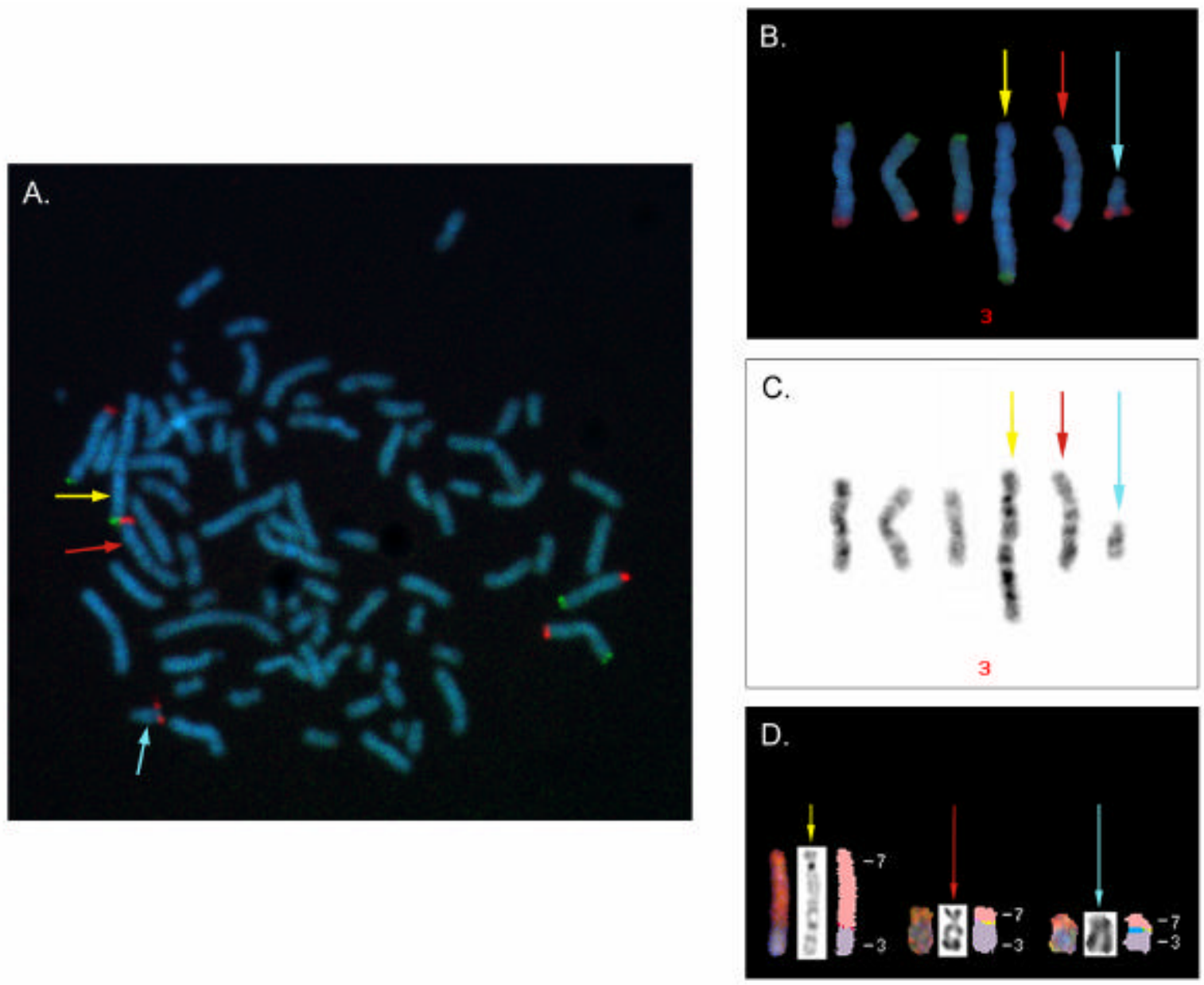

Figure 26. Visual Comparison of FISH and SKY. During the scoring of telomere probes $(3 p=$ green, $3 q=$ red) in interphase FISH of OCM-1, we happened upon a metaphase spread that was particularly illustrative regarding the amount and the arrangement of chromosome 3 telomere material. (A) A digital image of the metaphase spread, where the yellow, red, and blue arrows indicate the 3 aberrant chromosomes. (B) All of the chromosome 3 material in the metaphase spread is grouped following image processing. (C) Inversion of the DAPI images for the chromosomes in (B) revealed a banding pattern that allowed accurate determination that these examples from FISH and SKY (D) were equivalent. Analysis reveals there are 2 normal copies of chromosome 3, a third potentially abnormal copy, a der(3p;7) (yellow arrow), a der(3q;7) (red arrow), and a truncated copy of $3 \mathrm{q}$ (blue arrow). 
regions were those copy number abnormalities identified in greater than $30 \%$ but fewer than $70 \%$ of all tumor cases. Any copy number abnormality seen in less than $30 \%$ of the cases is likely not a strong indicator of prognosis. Conversely, those abnormalities present in greater than $70 \%$ of the cases are not of prognostic significance, since they are likely commonalities among all cases of uveal melanoma rather than predictive of outcome.

Within the minimal common regions, differential gain and loss was observed between male and female cases on the sex chromosomes (Figs. 28 and 29). The data was further subdivided into minimal common regions for those who survived 9 years or more post-diagnosis, and those that succumbed to the disease in that time frame (Figs. 30 and 31). Additionally, the sex chromosomes were evaluated independently for male and female subsets within the live and dead cohorts (Fig. 32). The 25 male patients who succumbed to the disease evidenced a $+\mathrm{Xq} 21$ not seen in the 21 male patients who survived beyond 9 years post-diagnosis, but no difference was seen on the $\mathrm{X}$ chromosome in the female cases (Fig. 32).

Statistical comparison of the live and dead data sets revealed six chromosomal regions that correlated strongly with a poor prognosis; five evidenced gain (18q11.2, 6q16, 21q11.2, 9q12 and 3q12), and one showed a loss (1p33). Overall, the strongest correlation with prognosis was associated with gain of $18 \mathrm{q} 11.2(\mathrm{p}=0.0037)$ (Fig. 33). Gain of 18q11.2 and concomitant loss of $1 \mathrm{p} 33$ was the strongest multivariate predictor of prognosis $(\mathrm{p}=0.0028)($ Fig. 34) 

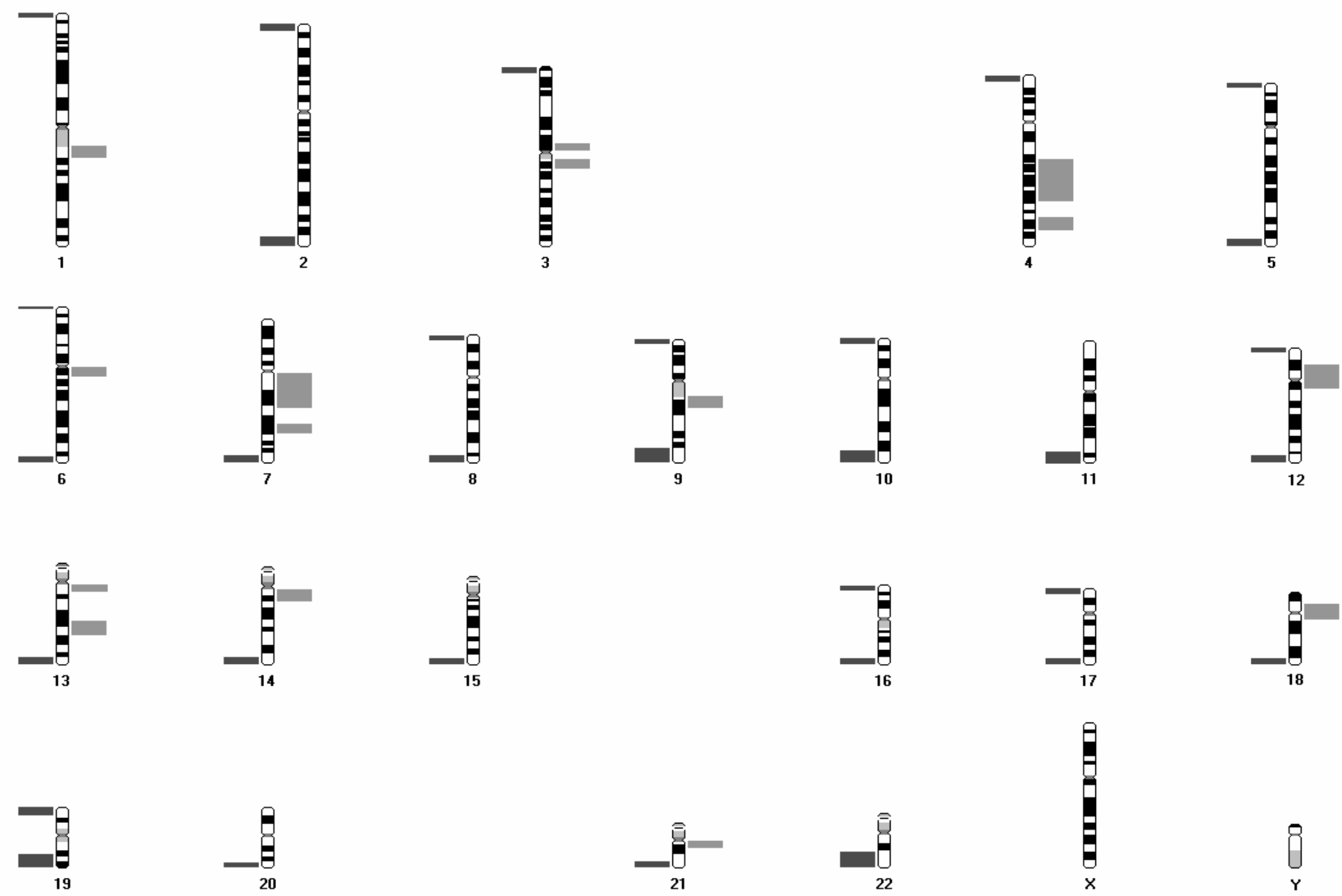

18

Figure 27. Minimal Common Regions of Gain and Loss Distilled from CGH Mean Profile Data of 82 FFPE Uveal Melanoma Cases. The light gray bars to the right of the ideogram represent gain, and dark gray bars to the left of the ideogram represent regions of loss, common between more than $30 \%$ and less than $70 \%$ of the archival cases. These minimal common regions may denote chromosomal regions related to the development and progression of uveal melanoma. Minimal common regions for the sex chromosomes are provided in Figs. 28, 29 and 32. 
A. Chromosome X Data for 46 cases

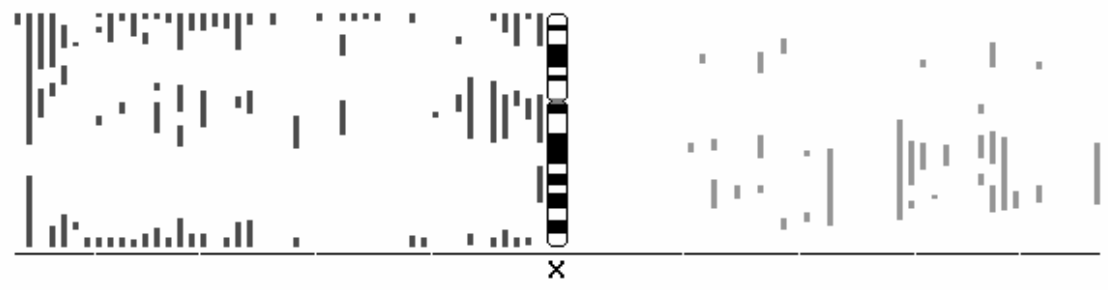

Chromosome X MCR

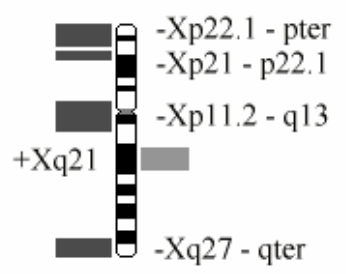

B. Chromosome Y Data for 46 Cases

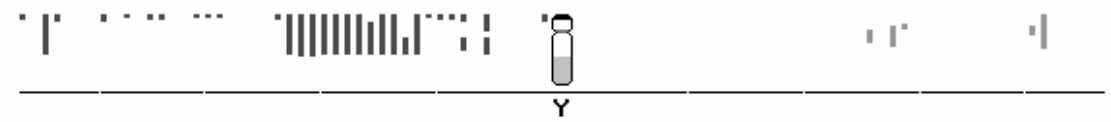

\section{Chromosome Y MCR}

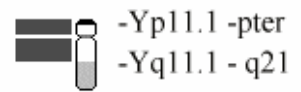

Figure 28. Quantitative Comparison Data and Minimal Common Regions for the Sex Chromosomes of the Male Archival Cases. (A) The QPD for the X chromosome is shown above the minimal common regions for hat chromosome. Regions of gain and loss, possibly those having prognostic significance, are shown in the minimal common regions. (B) The QPD and the minimal common regions are shown for the $\mathrm{Y}$ chromosome. 
FEMALE SEX CHROMOSOME DATA

A. Chromosome X Data for 36 Cases

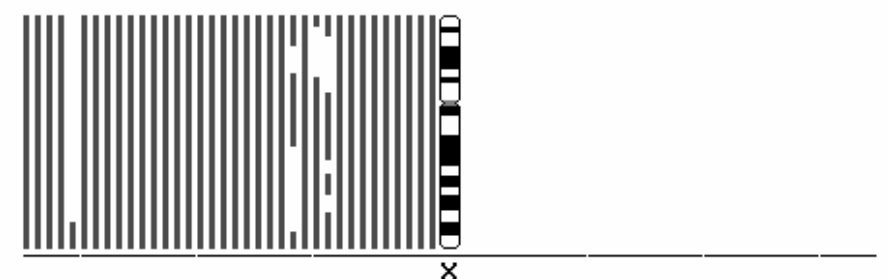

Chromosome X MCR

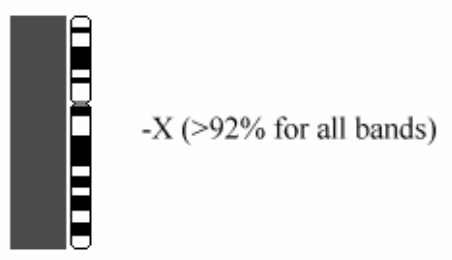

B. Chromosome Y Data for 36 Cases

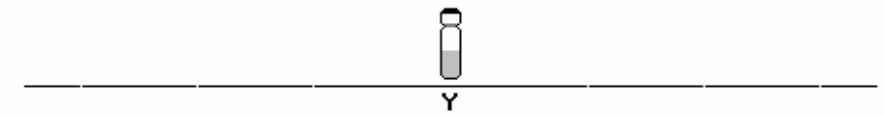

\section{Chromosome Y MCR}

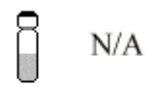

Figure 29. Quantitative Comparison Data and Minimal Common Regions for the Sex Chromosomes of the Female Archival Cases. (A) The QPD for the X chromosome is shown above the minimal common regions for that chromosome. In greater than $92 \%$ of the cases, the entire $\mathrm{X}$ chromosome was lost, eliminating it from having any prognostic significance. (B) The QPD and the minimal common regions reveal no gains or losses, as expected, in the female cases. 

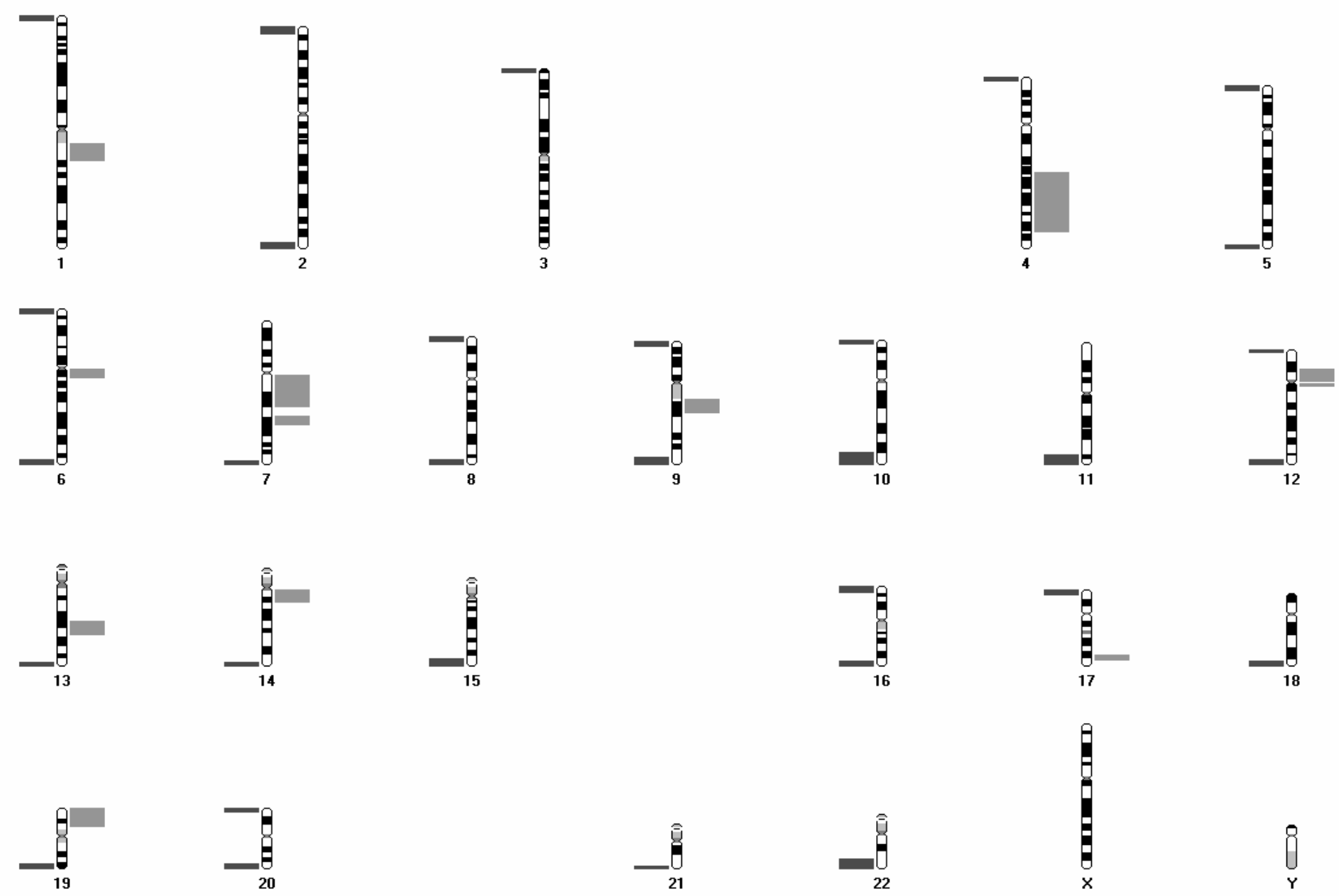

Figure 30. Minimal Common Regions of Gain and Loss Distilled from CGH Mean Profile Data of 51 FFPE Uveal Melanoma Cases, Representing Those Patients that Survived Beyond 9 Years Post-diagnosis. The light gray bars to the right of the ideogram represent gain, and dark gray bars to the left of the ideogram represent regions of loss, common among more than $30 \%$ and less than $70 \%$ of the archival cases. These minimal common regions may denote chromosomal regions related to a more favorable prognosis in uveal melanoma. Minimal common regions for the sex chromosomes are provided in Figs. 28, 29 and 32. 

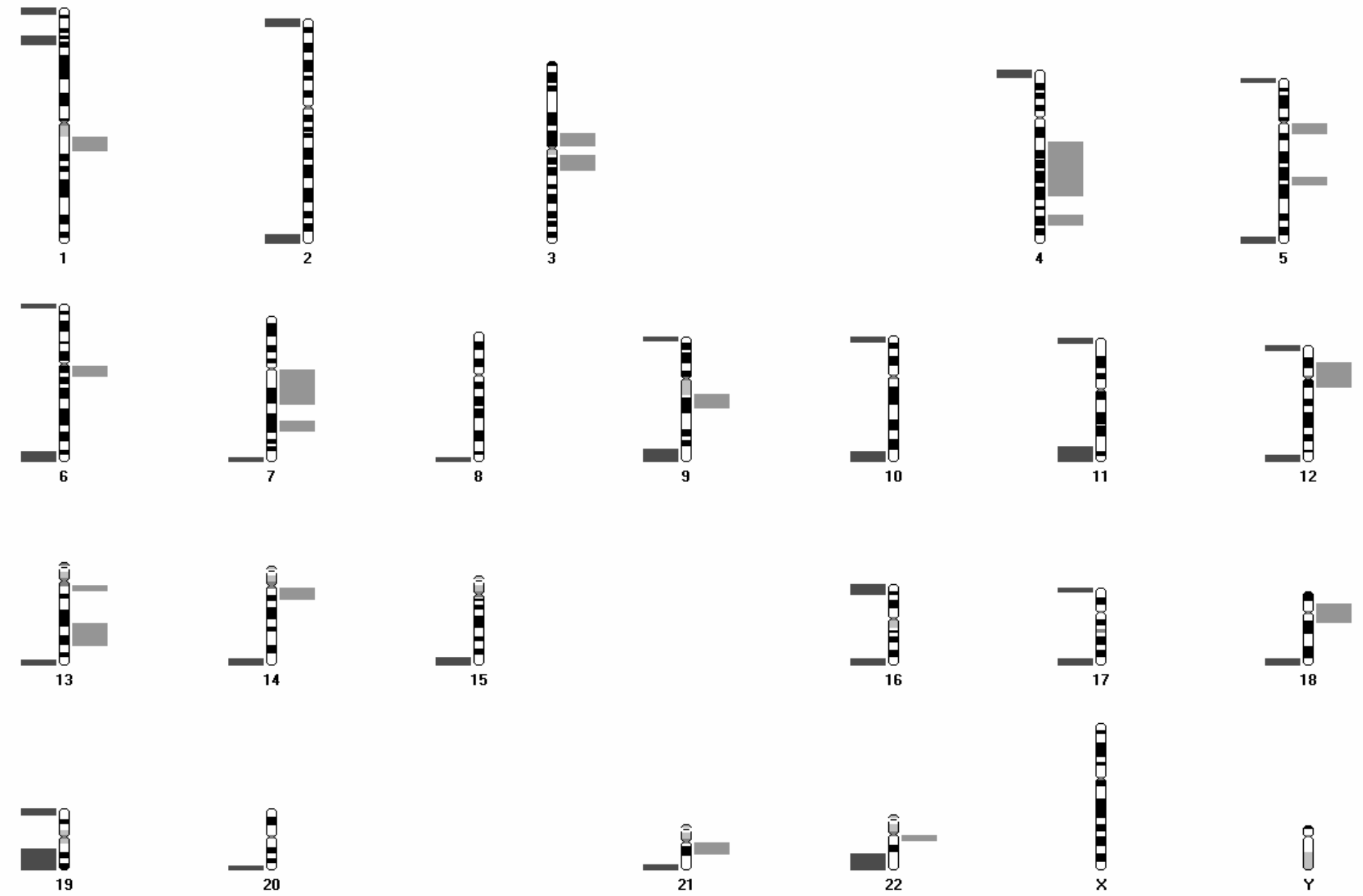

Figure 31. Minimal Common Regions of Gain and Loss Distilled from CGH Mean Profile Data of 49 FFPE Uveal Melanoma Cases, Representing Those Patients that Succumbed to Hematogenous Metastasis. The light gray bars to the right of the ideogram represent gain, and dark gray bars to the left of the ideogram represent regions of loss, common between more than $30 \%$ and less than $70 \%$ of the archival cases. These minimal common regions may denote chromosomal regions related to a poor prognosis in uveal melanoma. Minimal common regions for the sex chromosomes are provided in Figs. 28, 29 and 32. 


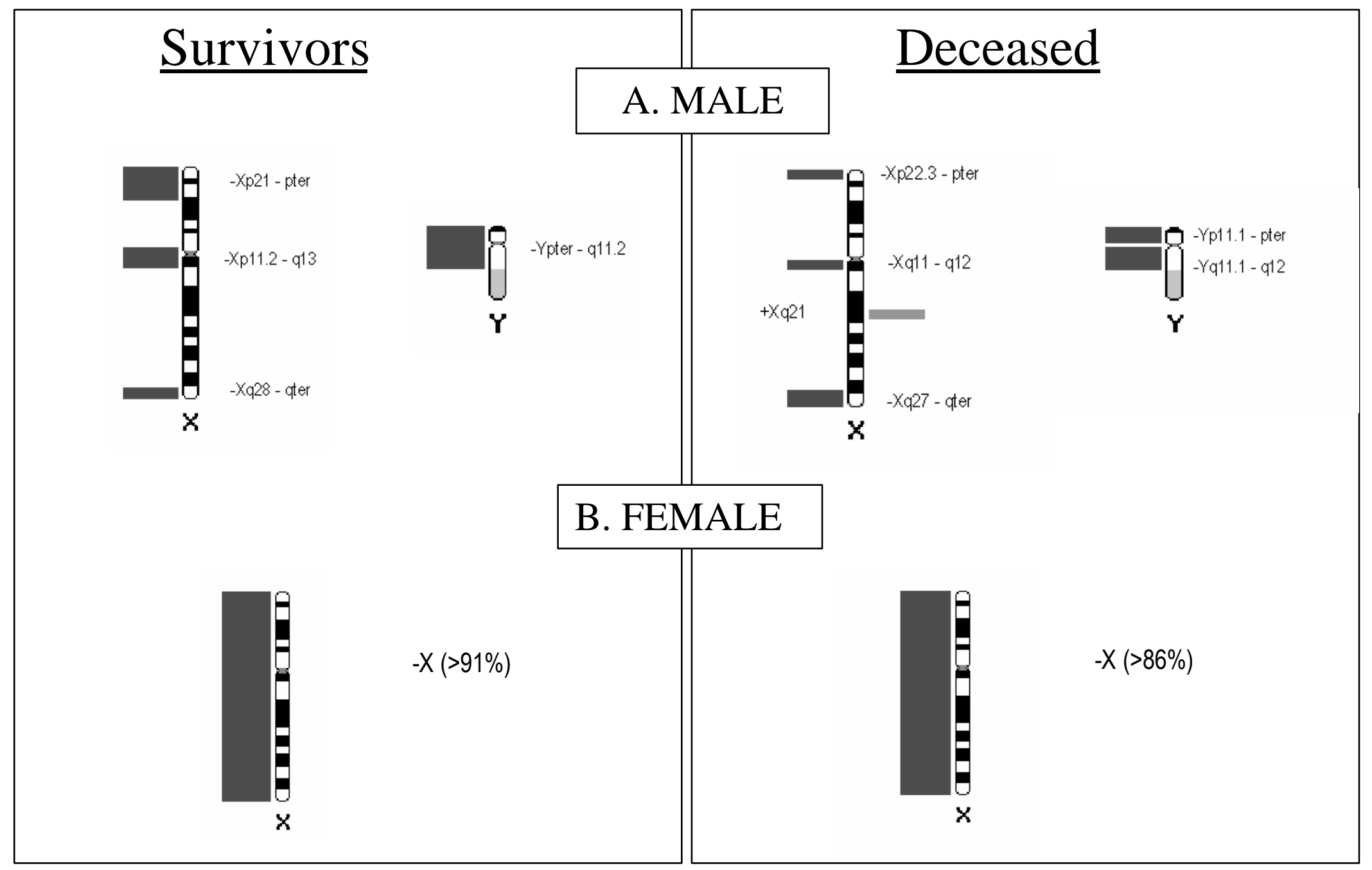

Figure 32. Minimal Common Regions for the Sex Chromosomes, Separated by Gender and Survival. (A) The sex chromosomes for males that survived beyond 9 years with uveal melanoma ( 23 cases) are compared with the male cases that succumbed to hematogenous metastasis (29 cases). (B) The X chromosome is compared for the female cases that survived beyond 9 years (28 cases) and those females that succumbed to the disease within that time period (20 cases). 


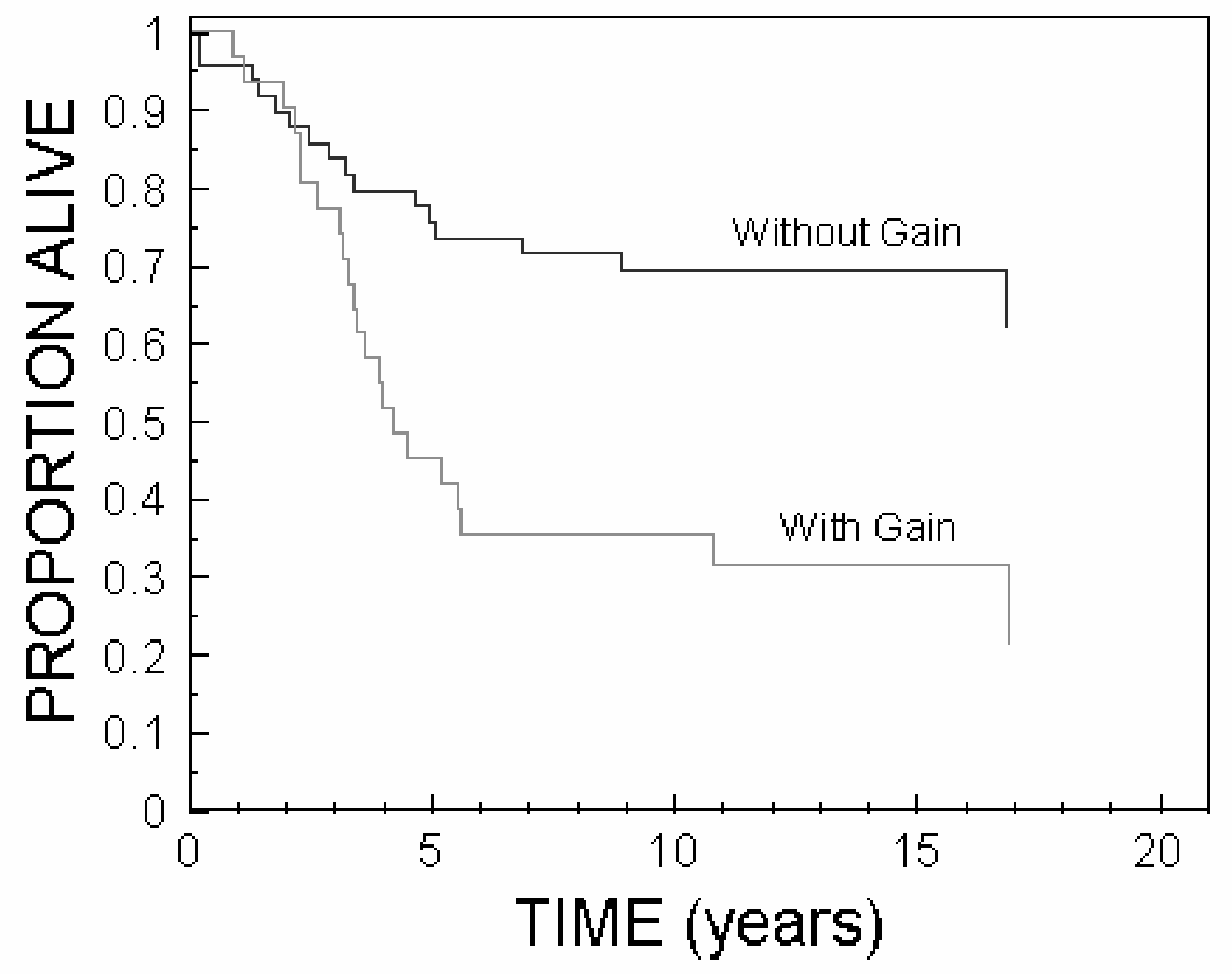

Figure 33. Univariate Kaplan-Meier Cause Specific Survival Correlation Curve. This comparative plot shows the two-fold negative effect on survival that is conferred by the gain of 18q11.2. This plot was generated from results comprised of all 82 FFPE archival cases in our CGH analysis. 


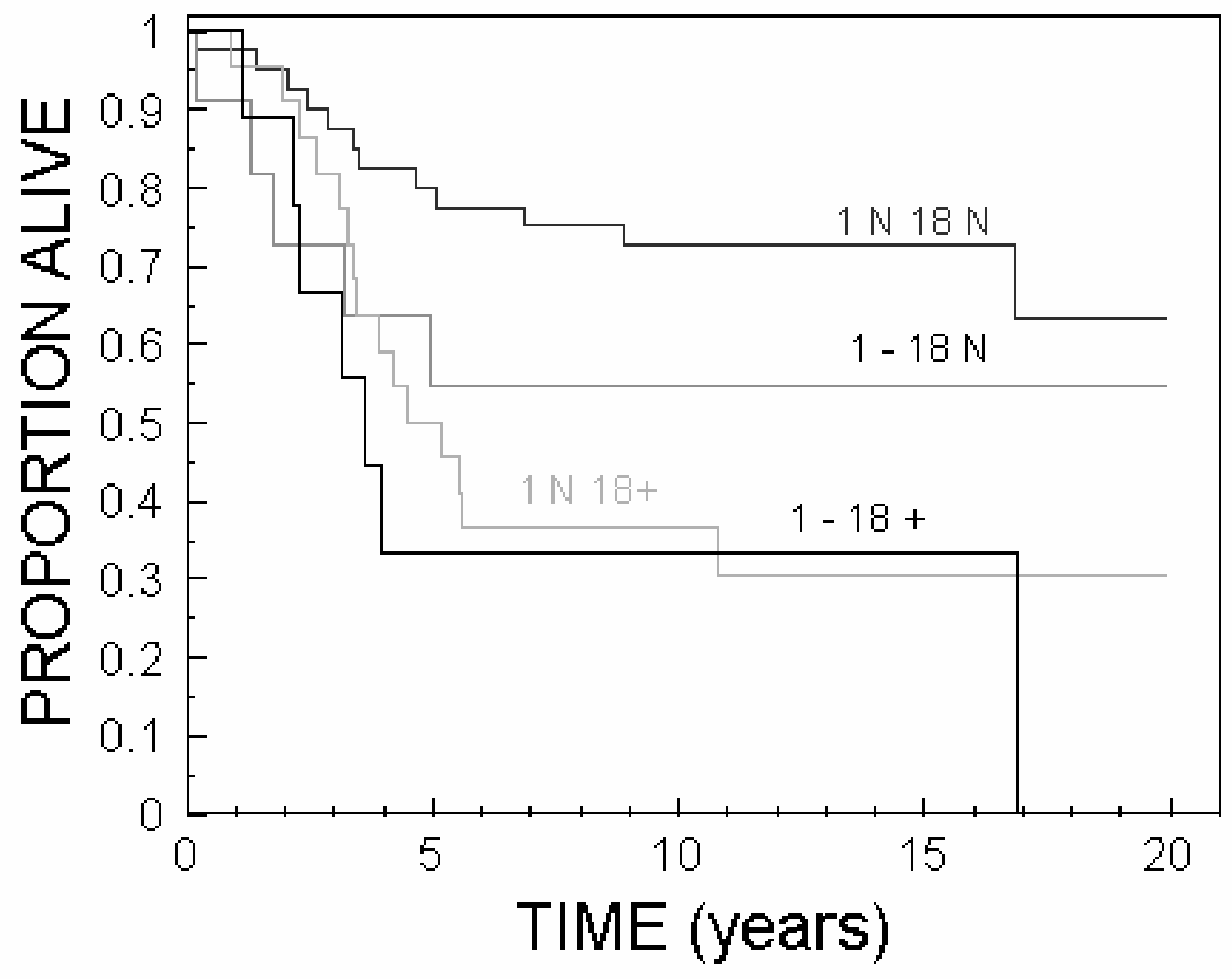

Figure 34. Multivariate Kaplan-Meier Cause Specific Survival Correlation Curve. This comparative plot shows the cumulative negative effect on survival that is conferred by the gain of 18q11.2 and concomitant loss of $1 \mathrm{p} 33$. This plot was generated from results comprised of all $82 \mathrm{FFPE}$ archival cases in our CGH analysis. 


\section{DISCUSSION}

Chromosomal rearrangements, both numerical and structural, are often correlated with morphology, tumor stage, and the aggressive potential of the tumor; therefore, such aberrations are often of prognostic value (Rothmann et al., 1998). Some of the chromosomal rearrangements associated with uveal melanoma have been identified, but often without being linked to a particular cellular function. Furthermore, the majority of studies thus far have looked at relatively small populations, and employed cytogenetic techniques with fairly low resolution. There remains a need for large-scale profiling of the genomic changes in uveal melanoma, using higher resolution methodologies, in order to increase and better define the catalogue of cytogenetic aberrations associated with the disease. Complete cytogenetic characterization of uveal melanoma is a critical step toward gene-specific investigation of malignancy.

To that end, we fully characterized 10 uveal melanoma cell lines and 82 archival FFPE tumors using molecular cytogenetic techniques. In our ell line study, CGH revealed genomic imbalances, and SKY provided insight into numerical and structural abnormalities that contribute to these imbalances in the genome. The tandem application of CGH and SKY, and the associated synthesis of the results, allowed for a comprehensive analysis of chromosomal abnormalities in the genome and insight into the mechanisms of chromosomal aberrations that have accumulated during clonal divergence (Veldman et al., 1997; Macville et al., 1999). We applied a third technique, FISH, to our cell lines to assay three specific loci as a practical means of chromosome 3 enumeration. In our archival tissue study, a thorough evaluation of 82 primary uveal melanoma tumors, culled from the tissue archives of the AFIP, revealed the specific genomic imbalances 
associated with each tumor, and helped determine regions predictive of prognosis. These 2 studies helped to more fully understand the underlying cytogenetic events of uveal melanoma development and progression.

\section{Cell Line S Tudy.}

Our research demonstrated that synthesis of CGH and SKY techniques develops an integrated data set comprised of numerical and structural alterations. Utilizing this approach, copy number aberrations were localized in chromosomal regions of the 10 cell lines. These specific regions are likely to contain genes differentially expressed in uveal melanoma.

CGH provided a comparison of the cell line genomes through evaluation of mean profile data (Fig. 19), and revealed commonalties in the data set, recorded as minimal common regions (Fig. 20). These regions are either gained or lost in 50\% or more of the cell lines, and are likely of significance in uveal melanoma. It is thought that within these regions are the genes that, when their copy number is altered, contribute to the establishment and/or progression of uveal melanoma.

The definitive numerical and structural composition of individual cell line genomes was revealed by SKY, and evidence of rearrangement was present within the chromosome complement of each cell that was analyzed. Reconciliation of multiple single cell analyses in our SKY results provided an adequate representation of the genome of each cell line. Comparison of SKY results between the cell lines identified five common chromosomal abnormalities, each seen in a minimum of four cell lines (Fig. 22). The prevalence of these aberrations suggests that they are of import to uveal melanoma development or progression. Our comprehensive SKY analysis revealed 
extensive numerical changes and structural rearrangements in each cell line, which resulted in aneuploidy, instances of mosaicism, aneusomy and partial chromosome amplifications and deletions.

Before we could interpret our findings from $\mathrm{CGH}$ and SKY, we needed to determine the correlation between the two techniques. However, there was a caveat to our interpretation of the $\mathrm{CGH}$ and SKY results. A known limitation of $\mathrm{CGH}$ is the inability to elucidate structural information, as only numerical data are gleaned from the compilation and analysis of mean ratio profiles. Similarly, SKY can not be expected to reveal all permutations of structural and numerical rearrangement in a cell line, as the analysis is based on a few analyzable metaphase spreads. With that in mind, we were able to isolate multiple visual examples where CGH and SKY showed agreement regarding chromosomal aberrations within a given cell line (Fig. 23). SKY results strongly demonstrate that the gains and losses identified by $\mathrm{CGH}$ are not exclusively due to numerical chromosome aberrations. Instead, it is clear that structural rearrangement of the genome also significantly contributes to amplification and deletion of DNA sequences. Complementation between SKY and CGH data is exemplified in the formation of an isochromosome $6 p$ and concomitant loss of a $6 q$ in MEL270, which yielded four copies of $6 p$ and only 2 copies of the $6 q$ arm (Fig. 24). This was mirrored in the $\mathrm{CGH}$ results that show a gain of $6 \mathrm{p}$ and loss of $6 \mathrm{q}$ for that cell line. Also shown in Figure 24, are additional examples in which genomic imbalances detected by CGH are mirrored in the SKY analysis from the same cell lines.

An additional feature of SKY, is its ability to resolve complex chromosomal rearrangements (Fig. 21). While these more complex rearrangements also affect copy 
number, it is not possible to resolve this by a simple visual inspection of results. Therefore, we developed mathematical calculations of genomic balance for CGH and SKY that could be statistically correlated. This quantitative approach for comparing techniques also served as an independent confirmation of the visual correlation seen between CGH and SKY.

While visual confirmation of the link between CGH and SKY was intriguing, demonstration of statistical correlation between the 2 techniques would lend credence to our observations. Unfortunately, SKY results for OCM-3 were compromised in the data recovery process, and were omitted from statistical analysis. Comparison of the relative balance for the 9 remaining cell lines, analyzed by both CGH and SKY, showed significant correlation across the entire genome, as determined by the associated $\mathrm{R}^{2}$ - and p-values (Fig. 23). Additionally, chromosome-specific correlations were drawn from CGH and SKY results for the chromosomes previously reported as having prognostic value for uveal melanoma (Fig. 25B-D and Table 7). The strength of these quantitative comparisons confirmed our visual correlations between the two independent in situ hybridization techniques, and validated our molecular cytogenetic characterizations of the cell line genomes.

The correlation established between CGH and SKY permitted further interpretation of the data sets. Several chromosomes $(6,7,17 q$ and $20 q)$ that showed imbalance of large continuous sections by CGH in at least half of the cell lines were also involved in frequent numerical or structural aberrations revealed by SKY (Fig. 20 and Table 5). The involvement of chromosomes 6 and 7 in uveal melanoma is well documented in the literature (Gordon et al., 1994; Speicher et al., 1994; Ghazvini et al., 
1996; White et al., 1998b). Our CGH and SKY results show copy number alteration events on other chromosomes similar to those in the literature (Tables 2 and 3) (Wiltshire et al., 1993; Gordon et al., 1994; Speicher et al., 1994; Ghazvini et al., 1996; Prescher et al., 1996; White et al., 1998a and b). It should be noted that both CGH and SKY identified alterations of $17 \mathrm{q}$ and $20 \mathrm{q}$, neither of which has been previously implicated in uveal melanoma. We suggest that these chromosomes warrant further study to determine their significance. The comparison of our data with the published literature suggests our demonstrated lack of monosomy 3 by CGH or SKY is most noteworthy and requires explanation.

Application of FISH in a confirmatory capacity following CGH or SKY is a very powerful application of the technique (Macville et al., 1999). We used human chromosome 3-specific centromere and telomere FISH probes on interphase preparations of all 10 cell lines to confirm our CGH and SKY results for chromosome 3. Our 3cen hybridizations seemed to indicate loss of chromosome 3 , as the dominant population in each cell line had a single 3cen hybridization signal per nucleus, but the range spanned from 0 to 6 signals. In contrast, the number of telomere probe hybridization signals ranged from 0 to 12 signals, with a tendency for 3 or more signals per telomere in each nucleus. These results indicated a high degree of heterogeneity in the cell lines, and vastly different chromosome 3 complements than our diploid control (Table 6). Heterogeneity was also observed in cell line karyotypes from our SKY analyses. The lack of a major clone in the cell line populations, coupled with the high degree of heterogeneity observed by SKY and FISH, is suggestive of mosaicism in these cell lines. The signal variation and enhanced copy numbers for the centromere and telomere probes, 
suggested the presence of enhanced copies of portions of chromosome 3 . This is also shown in the CGH profiles for each cell line, and the numerous rearrangements bearing chromosome 3 material in our SKY analyses (Figs. 19 and 22E). The distribution of chromosome 3 material observed in SKY and the partial chromosome 3 gains in $\mathrm{CGH}$, when considered alongside the FISH results, point toward rearrangement as playing a key role in chromosome 3 involvement. Visual confirmation of the role of rearrangement came from a comparison between FISH and SKY findings that show enhanced copy number for chromosome 3 and define specific rearrangements associated with OCM-1 (Fig. 26). Further, chromosome-specific correlations show that chromosome 3 rearrangement contributes to copy number enhancement (Fig. 25A and B). Ideally, all three probes (3cen, 3p and 3q) would have been simultaneously hybridized to determine the precise assortment of each signal. This would have provided more insight into the precise mechanism(s) of rearrangement, and also definitively pinpointed mosaicism, but the hybridization was not technically feasible.

Although monosomy 3 has been heavily reported as a hallmark for uveal melanoma, our findings for chromosome 3, confirmed using FISH following independent CGH and SKY analyses, clearly indicate there was no loss of a whole chromosome 3 in these cell lines. In contrast to our methodologies, the majority of uveal melanoma analysis published in the literature is derived from classical cytogenetic evaluations (Horsman et al., 1990; Sisley et al., 1990; Prescher et al., 1990; Horsthemke et al., 1992; Sisley et al., 1992; Horsman and White, 1993; Wiltsire et al., 1993; Singh et al., 1994; Prescher et al., 1994; Prescher et al., 1995; Prescher et al., 1996; White et al., 1998b; Parada et al., 1999; Sisley et al., 2000; Tschentscher et al., 2001). However, other 
studies have used molecular cytogenetic methods in their determination of monosomy 3 , which indicates that our findings are not linked to our methodologies (Ghazvini et al., 1996; Prescher et al., 1996; McNamara et al., 1997; Sisley et al., 1997; Tschentscher et al., 2001). The absence of chromosome 3, 6, and 8 abnormalities in some cases, suggests there are additional pathways of malignant transformation (Singh et al., 1996a). Thus, there were indications that there could be alternate explanations for our findings on chromosome 3.

The search for alternative explanations for our lack of monosomy 3 , led to an examination of cell line origin. Monosomy 3 is frequently reported in tumors of the ciliary body, whereas a normal or polysomic complement of chromosome 3 is typically reported in choroidal melanoma (Sisley et al., 1993; McNamara et al., 1997). Our information regarding cell line origin is incomplete (Table 1), but available information shows only one cell line (MEL270) was established from a ciliary body tumor. A second cell line (OMM2.3) was established from a metatstasis of the same primary tumor. Neither MEL270 nor OMM-2.3 evidenced any loss on chromosome 3 by CGH, while OMM-2.3 actually demonstrated significant gains on chromosome 3. In fact, only one cell line (MEL285) demonstrated a considerable loss of chromosome 3 material, and three other lines (MEL290, OCM-3 and OCM-8) lost only distal 3p material (Fig. 19). The origin of these four cell lines was indeterminate, which effectively prevented further correlation between CGH results and cell line origin. SKY results revealed truncated or partially deleted copies of chromosome 3 in at least three of our cell lines (OCM-3, OCM-8 and OM431), in addition to normal copies of chromosome 3. The retention of abnormal copies of chromosome 3 precludes the designation of monosomy to any of 
these cell lines. Further, FISH results indicate different mean signal counts for each probe within a cell line, which is not congruent with a determination of monosomy (Table 6).

Additional investigation regarding the original karyotype, specifically a closer look at chromosome 3, demonstrated strong parallels between our findings and those published at the time of establishment. Although establishment karyotypes were only available for a subset of the cell lines, this investigation was warranted because our cell lines were of an indeterminate number of passages, and verification of our findings strengthened any conclusions regarding chromosome 3. The published karyotype for OCM-1 includes $\operatorname{add}(3)(q 12)$ and $+\operatorname{del}(3)(q 13 q 23)$ (Luyten et al., 1996). The majority of chromosome 3 was demonstrated to be gained in OCM-1 via CGH, with the exception of 3q13-q23 and distal 3p (Fig. 19). Also, multiple truncated copies of chromosome 3 were found in our SKY results for OCM-1. The karyotype for OMM-1 indicated a $+3[50 \%]$ at the time of establishment, but our CGH results revealed only a $3 p$ gain, and SKY revealed an apparent trisomy 3 or possibly an isochromosome 3p (Luyten et al., 1996). Meanwhile, the cell line 92-1 had no known chromosome 3 abnormalities at the time the cell line was established (DeWaard-Siebinga et al, 1995). There was evidence of a very small focal 3q13.3 gain in 92-1, and no losses reported along the length of the chromosome (Fig. 19). Our findings for chromosome 3 demonstrate minimal divergence from the original karyotype for these 3 cell lines, and the strength of our quantitative analyses suggests the same would hold true for the remainder of the cell lines (Figs. 23 and 25). 
No explanation for the lack of monosomy 3 was found in the cell line origin, and there were no indications were that our cell lines had significantly diverged while in culture. Based on the strength of the correlations determined for our three techniques, we sought to reevaluate the literature to elucidate possible discrepancies in data interpretation between our study and others. Some published reports in the literature, based upon CGH analyses, have noted a "decreased copy number" for chromosome 3 in lieu of monosomy. These results provide evidence of a copy number below 1 , but the profile does not cross the loss threshold (Gordon et al., 1994; Ghazvini et al., 1996). Others have alluded to two tumor suppressor loci, one on the p- and a second on the qarm of the chromosome, which result in focal deletions on chromosome 3 instead of whole chromosome loss (Sisley et al., 1993; White et al., 1998a; Parella et al., 1999; Myatt et al., 2000; Tschentscher et al., 2001). In one LOH study, two purported tumor suppressor loci were defined in terms of the smallest region of overlap (SRO), one within band 3p25 and the other on the q-arm between 3q24-q26 (Tschentscher et al., 2001). A second study implicated only 3p24.3 by LOH (Sisley et al., 1993). Two more LOH studies demonstrated isodisomy of chromosome 3, which may lead to functional monosomy (White et al., 1996; White et al., 1998a). Comparison of results from CGH and LOH studies typically results in a high degree of concordance (Ried et al., 1997). Therefore, the alignment of our findings with those of LOH studies is not surprising. Elsewhere in the literature, a GTG-banding study reported loss of 3p13 (Blasi et al., 1999). A later study by Naus and colleagues, who also applied CGH, SKY and FISH to characterize uveal melanoma, failed to find monosomy 3 but were able to define $3 q 13$ q21 as a region of loss (Naus et al., 2001). The findings of Blasi et al. (1999) and Naus 
et al. (2001) suggest a region near band 3q13 may potentially harbor a tumor suppressor gene. Other findings in the literature, including trisomy 3 and even tetrasomy 3 as reported by McNamara and colleagues, are supportive of our CGH, SKY and FISH results that indicate gains on chromosome 3 (Figs. 16, 22 and 23, Tables 6 and 7) (McNamara et al., 1997). This review of the literature revealed indications that there are explanations for the involvement of chromosome 3 other than whole chromosome loss.

Another finding of note was the consistent involvement of several specific chromosomes in structural rearrangements as seen by SKY, which suggests a redundant mechanism for this occurrence (Fig. 22, Table 5). Our cell lines were shown to have minimally diverged in culture, therefore cell culture artifacts only account for a small proportion of these findings. Each of the five common chromosomal interactions observed across multiple cell lines, which include several chromosome 3 interactions, involved chromosomes with deletions in one or both telomeric regions of the chromosome (Figs. 19 and 22). Other telomeric deletions in specific cell lines were related to increased structural rearrangement of those same chromosomes in SKY analysis, and these deletions on the distal p- or q-arms were consistent with the rearrangement observed in the SKY karyotypes. In general, chromosomes shown to evidence telomeric deletion in the minimal common regions, also revealed higher frequencies of structural rearrangements by SKY (Fig. 20 and Table 5). This suggests that telomeric loss may facilitate structural rearrange ment, but further study would be required to accurately determine the significance of this finding in the uveal melanoma cell lines. 
CGH and SKY represent distinct approaches that reveal complementary information on cytogenetic features of uveal melanoma. Utilizing a combination of CGH, FISH and SKY we confirmed the absence of monosomy 3 in these ten cell lines, and demonstrated alternative manifestations of chromosome 3 in this tumor. Our findings do not refute the occurrence of monosomy 3 reported by others, but instead we postulate that frequent rearrangement of chromosome 3 may have an equally important contribution in the mechanisms driving uveal melanoma. The portion of the study that followed, a study of archival tissue by $\mathrm{CGH}$, was expected to enhance resolution of the critical chromosomal regions throughout the genome and ultimately aid in the determination of specific gene involvement in uveal melanoma.

\section{TUMOR STUDY.}

Classification of tumors based on histopathology and tumor grading is subjective and qualitative, based on a two-dimensional image analysis (Rothmann et al., 1998). Classical cytogenetic studies, while useful in elucidating structural abnormalities, are time consuming and require fresh tissue. Furthermore, classical cytogenetic analysis is compromised when dealing with solid tumor preparations due to low mitotic indices, poor growth in culture, etc. (Joos et al., 1994; Houldsworth and Chaganti, 1994; Isola et al., 1994; Macville et al., 1997; Veldman et al., 1997; Ried et al., 1997; Rothmann et al., 1998). Techniques such as CGH are expected to yield more information. Our large-scale CGH study, aimed at determining a set of prognostic indicators in uveal melanoma, identified specific regions of gain and loss (Figs. 27, 28 and 29). These regions were initially derived irrespective of the survival correlation portion of the study, thus indicating regions that may contain genes leading to development of uveal melanoma. 
The CGH data was further subdivided based upon survival correlation (Figs. 30, 31 and 32). Regions containing genes associated with malignancy were revealed in the survival correlation and associated minimal common regions, and are presumed to be indicators of prognosis. Statistical analyses confirmed 6 regions of import with regard to survival. Of these, the most potent predictor of prognosis identified in this study was 18q11.2 (Fig. 33). Multivariate analyses revealed that gain of 18q11.2 combined with the loss of 1p33 had the most drastic effect on prognosis (Fig. 34).

Application of a Bonferroni correction obfuscates the statistical significance of our findings. This includes both conservative and rigorous applications of this correction. This is likely attributable to a relatively small sample size, further subdivided for survival correlation analysis, for such an application.

There is evidence that suggests gender may be involved in uveal melanoma susceptibility, as males are slightly more susceptible than females (McLean and Gamel, 1996). CGH results revealed very different regions of gain and loss on the $X$ chromosome in a comparison of male and female cases (Figs. 28 and 29). The minimal common regions revealed that $+\mathrm{Xq} 21$ is unique to male patients who succumbed to uveal malignant melanoma (Figs. 28, 29 and 32). This region was not statistically significantly different in the analysis, because of the reduction of the sample size required to simultaneously analyze survival and gender (Fig. 32), but may warrant further study.

Due to the high frequency with which chromosome 3 loss is reported in uveal melanoma, data interpretation of CGH becomes critical in evaluation of chromosome 3 . A closer look at the published literature revealed some inconsistencies. In some reports, $\mathrm{CGH}$ research has noted a decrease in copy number of chromosome 3 instead of whole 
chromosome loss (Gordon et al., 1994; Ghazvini et al., 1996). In such instances, the ratio profile indicates a copy number ratio below 1 , but does not exceed the loss threshold in all cases. These results indicate a tendency toward loss, rather than monosomy 3 as reported (Gordon et al., 1994; Ghazvini et al., 1996). Chromosome 3 findings in previously published uveal melanoma studies have revealed contributions of chromosome 3 other than monosomy. LOH studies have demonstrated loss on either the p- or q-arm of the chromosome, but not complete loss along the length of the chromosome (White et al., 1996; White et al., 1998a; Parella et al., 1999). Other LOH studies have localized various regions of loss to $3 \mathrm{p} 24.3,3 \mathrm{p} 13$, 3p22 (TGFbetaR2 gene locus), 3p25, and 3q24-q26 (Sisley et al., 1993; Blasi et al., 1999; Myatt et al., 2000; Tschentscher et al., 2001).

We did not find any instances where the entire CGH ratio profile exceeded the threshold for loss. All partial chromosome losses, or tendencies toward loss, were recorded on interstitial portions of the $\mathrm{p}$ and $\mathrm{q}$-arms. Any observations in the most proximal and most distal bands of chromosome 3 were not included in this segment of the analysis, due in large part to the variability in hybridization near the centromere or telomere. The observed regions of interstitial loss fall within 3p13-p25 and 3q12-q28. Findings in other studies, particularly involving $\mathrm{LOH}$ of chromosome 3, align with our findings.

A drawback to large-scale CGH is the potential to mask critical chromosomal regions present in a low frequency within a given sample or within a collection of samples, and a similar drawback is realized in the distillation of minimal common regions. However, correlation of $\mathrm{CGH}$ with patient follow-up, using a variety of 
established prognostic factors, has more utility. Using this approach, we have found six regions shown to be of significance to prognosis, including 18q11.2, 6q16, 21q11.2, 9q12, 3q12, and 1p33. We also identified a region on the $X$ chromosome that seemed to correlate with a poor prognosis in males, but was not confirmed as statistically significant. All seven of these regions warrant further study. 


\section{SUMMARY}

Our complete characterization of 10 cell lines, and analysis of copy number imbalances in 82 archival specimens, represents the most extensive and comprehensive cytogenetic characterization of uveal melanoma to date. In our cell lines, we catalogued numerous numerical and structural chromosomal aberrations. Many of these aberrations were suggested by the genomic imbalances revealed in the study of cell line and archival material. Chromosomes 1, 3, 7, 12, 17 and 20 were most frequently involved in numerical or structural aberrations in our SKY analysis, and CGH confirmed substantial genomic imbalance on each of these chromosomes, suggesting an integral role for these

chromosomes in uveal melanoma. We were able to determine the involvement of chromosome 3 in our cell lines, and confirmed that rearrangement of that chromosome is more prevalent than whole chromosome loss in our samples. Our tumor study identified several chromosomal regions associated with prognosis, including 18q11.2, 6q16, 21q11.2, 9q12, 3q12, and 1p33. Together, our studies are aimed at directing further gene-specific study of malignancy in uveal melanoma. 


\section{REFERENCES}

Albert DM, Ruzzo MA, McLaughlin MA, Robinson NL, Craft JL, Epstein J: Establishment of cell lines of uveal melanoma. Methodology and characteristics. Invest Ophthalmol Vis Sci 1984, 25:1284-1299.

Bauman JG, Wiegant J, Borst P, van Duijn P: A new method for fluorescence microscopical localization of specific DNA sequences by in situ hybridization of fluorochrome labelled RNA. Exp Cell Res 1980, 128:485-490.

Bayani J and Squire JA: Advances in the detection of chromosomal aberrations using spectral karyotyping. Clin Genet 2001, 59:65-73.

Bayani JM and Squire JA: Applications of SKY in cancer cytogenetics. Cancer Invest 2002, 20:373-386.

Blasi MA, Roccella F, Ba lestrazzi E, Del Porto G, De Felice N, Roccella M, Rota R, Grammatico P: 3p13 region: a possible location of a tumor suppressor gene involved in uveal melanoma. Cancer Genet Cytogenet 1999, 108:81-83.

Blom DJ, Mooy CM, Luyten GP, Kerkvliet S, Ouwerkerk I, Zwinderman AH, Schrier PI, Jager MJ: Inverse correlation between expression of HLA -B and c-myc in uveal melanoma. J Pathol 1997, 181:75-79.

Callender GR: Malignant melanotic tumors of the eye: a study of histologic types in 111 cases. Transact Amer Acad Ophthalmol Otolaryngol 1931, 36:131-142.

Carpenter NJ: Molecular cytogenetics. Semin Pediatr Neurol 2001, 8:135-146.

Carter NP: Cytogenetic analysis by chromosome painting. Cytometry 1994, 18:2-10.

Casperson T, Farber S, Folley G, Kudynowski J, Modest E, Simonsson E, Wash U, Zech L: Chemical differentiation along metaphase chromosomes. Exp Cell Res 1968, 49:219-222.

Chana JS, Wilson GD, Cree IA, Alexander RA, Myatt N, Neale M, Foss AJ, Hungerford JL: c-myc, p53, and Bcl-2 expression and clinical outcome in uveal melanoma. Br J Ophthalmol 1999, 83:110-114.

Chang SS and Mark HF: Emerging molecular cytogenetic technologies. Cytobios 1997, 90:7-22. 
Chen PW, Murray TG, Uno T, Salgaller ML, Reddy R, Ksander BR: Expression of MAGE genes in ocular melanoma during progression from primary to metastatic disease. Clin Exp Metastasis 1997, 15:509-518.

Cohen IJ, Issakov J, Avigad S, Stark B, Meller I, Zaizov R, Bar-Am I: Synovial sarcoma of bone delineated by spectral karyotyping. Lancet 1997, 350:1679-1680.

Copeman, MC: The putative melanoma tumor-suppressor gene on human chromosome 6q. Pathology 1992, 24:307-309.

Cremer T, Landegent J, Bruckner A, Scholl HP, Schardin M, Hager HD, Devilee P, Pearson P, van der Ploeg M: Detection of chromosome aberrations in the human interphase nucleus by visualization of specific target DNAs with radioactive and non-radioactive in situ hybridization techniques: diagnosis of trisomy 18 with probe L1.84. Hum Genet 1986, 74:346-352.

Cremer T, Lichter P, Borden J, Ward DC, Manuelid is L: Detection of chromosome aberrations in metaphase and interphase tumor cells by in situ hybridization using chromosome-specific library probes. Hum Genet 1988, 80:235-246.

Dauwerse JG, Wiegant J, Raap AK, Breuning MH, van Ommen GJ: Multiple colors by fluorescence in situ hybridization using ratio-labelled DNA probes create a molecular karyotype. Hum Mol Genet 1992, 1:593598.

De Waard-Siebinga I, Blom DJ, Griffioen M, Schrier PI, Hoogendoorn E, Beverstock G, Danen EH, Jager MJ: Establishment and characterization of an uveal-melanoma cell line. Int J Cancer 1995, 62:155-161.

Demetrick DJ: Gone FISHin' for genes. CMAJ 1999, 161:1138.

Devilee P, Thierry RF, Kievits T, Kolluri R, Hopman AH, Willard HF, Pearson PL, Cornelisse CJ: Detection of chromosome aneuploidy in interphase nuclei from human primary breast tumors using chromosome-specific repetitive DNA probes. Cancer Res 1988, 48:5825-5830.

Dolin PJ, Foss AJ, Hungerford JL: Uveal melanoma: is solar ultraviolet radiation a risk factor? Ophthalmic Epidemiol 1994, 1:27-30.

du Manoir S, Speicher MR, Joos S, Schröck E, Popp S, Dohner H, Kovacs G, Robert-Nicoud M, Lichter P, Cremer T: Detection of complete and partial chromosome gains and losses by comparative genomic in situ hybridization. Hum Genet 1993, 90:590-610. 
du Manoir S, Kallioniemi OP, Lichter P, Piper J, Benedetti PA, Carothers AD, Fantes JA, Garcia-Sagredo JM, Gerdes T, Giollant M, Hemery B, Isola J, Maahr J, Morrison H, Perry P, Stark M, Sudar D, van Vliet LJ, Verwoerd N, Vrolijk J: Hardware and software requirements for quantitative analysis of comparative genomic hybridization. Cytometry 1995a, 19:4-9.

du Manoir S, Schröck E, Bentz M, Speicher MR, Joos S, Ried T, Lichter P, Cremer T: Quantitative analysis of comparative genomic hybridization. Cytometry 1995b, 19:27-41.

Dunne BM, McNamara M, Clynes M, Shering SG, Larkin AM, Moran E, Barnes C, Kennedy SM: MDR1 expression is associated with adverse survival in melanoma of the uveal tract. Hum Pathol 1998, 29:594598.

Eastmond DA and Pinkel D. Aneuploidy detection by analysis of interphase nuclei using fluorescence in situ hybridization with chromosome-specific probes. Prog Clin Biol Res 1989, 318:277-284.

Egan KM, Seddon JM, Glynn RJ, Gragoudas ES, Albert DM: Epidemiologic aspects of uveal melanoma. Surv Ophthalmol 1988, 32:239-251.

Fidler IJ: Critical factors in the biology of human cancer metastasis: twenty-eighth G.H.A. Clowes memorial award lecture. Cancer Res 1990, 50:6130-6138.

Forozan F, Karhu R, Kononen J, Kallioniemi A, Kallioniemi OP: Genome screening by comparative genomic hybridization. Trends Genet 1997, 13:405-409.

Gall JG and Pardue ML: Formation and detection of RNA-DNA hybrid molecules in cytological preparations. Proc Natl Acad Sci USA 1969, 63:378-383.

Garini, Y., Macville, M., du Manoir, S., Buckwald, R.A., Lavi, M., Katzir, N., Wine, D., Bar-Am, I., Schröck, E., Cabib, D., Ried, T: Spectral karyotyping. Bioimaging 1996, 4:65-72.

Garini Y, Gil A, Bar-Am I, Cabib D, Katzir N: Signal to noise analysis of multiple color fluorescence imaging microscopy. Cytometry 1999, 35:214-226.

Genetics of Cancer Resource Center, "Gbanded metaphase spread of a normal female somatic cell.” 2001, World Wide Web URL: http://www.intouchlive.com/cancergenetics/Images/b-banded-metaphase.jpg. 
Ghazvini S, Char DH, Kroll S, Waldman FM, Pinkel D: Comparative genomic hybridization analysis of archival formalin-fixed paraffin-embedded uveal melanomas. Cancer Genet Cytogenet 1996, 90:95-101.

Gordon KB, Thompson CT, Char DH, O'Brien JM, Kroll S, Ghazvini S, Gray JW: Comparative genomic hybridization in the detection of DNA copy number abnormalities in uveal melanoma. Cancer Res 1994, 54:4764-4768.

Griffin CA, Long PP, Schachat AP: Trisomy 6p in an ocular melanoma. Cancer Genet Cytogenet 1988, 32:129-132.

Herrington CS, Cooper K, McGee JO: Interphase cytogenetics: analysis of numerical chromosome aberrations in isolated cells. J Pathol 1995, 175:283-295.

Holly EA, Aston DA, Char DH, Kristiansen JJ, Ahn DK: Uveal melanoma in relation to ultraviolet light exposure and host factors. Cancer Res 1990, 50:5773-5777.

Hopman AH, Ramaekers FC, Raap AK, Beck JL, Devilee P, van der Ploeg M, Vooijs GP: In situ hybridization as a tool to study numerical chromosome aberrations in solid bladder tumors. Histochemistry 1988, 89:307-316.

Horsman DE, Sroka H, Rootman J, White VA: Monosomy 3 and isochromosome 8q in a uveal melanoma. Cancer Genet Cytogenet 1990, 45:249-253.

Horsman DE and White VA: Cytogenetic analysis of uveal melanoma. Consistent occurrence of monosomy 3 and trisomy 8q. Cancer 1993, 71:811-819.

Horsthemke B, Prescher G, Bornfeld N, Becher R: Loss of chromosome 3 alleles and multiplication of chromosome 8 alleles in uveal melanoma. Genes Chromosomes Cancer 1992, 4:217-221.

Houldsworth J and Chaganti RS: Comparative genomic hybridization: an overview. Am J Pathol 1994, $145: 1253-1260$.

Houlston RS and Damato BE: Genetic predisposition to ocular melanoma. Eye 1999, 13:43-46.

Hsu TC: Mammalian chromosomes in vitro. I. The karyotype of man. J Hered 1952, 43:167-172. 
Hungerford DA, Donnelly AJ, Nowell PC, Beck S: The chromosome constitution of a human phenotypic intersex. Am J Hum Genet 1959; 11:215-236.

Hungerford J: Uveal melanoma. Eur J Cancer 1993, 29A:1365-1368.

Institut für Pathologie, University Hospital Charité, "CGH Protocols." 1996, World Wide Web URL: http://amba.charite.de/cgh/protocol/02/prot02.html.

International RH Mapping Consortium, “GeneMap '99." 1999, World Wide Web URL: http://www.ncbi.nlm.nih.gov/genemap99.

ISCN (1995): An International System for Human Cytogenetic Nomenclature, Mitelman F (ed); S. Karger, Basel, 1995.

Isola J, DeVries S, Chu L, Ghazvini S, Waldman F: Analysis of changes in DNA sequence copy number by comparative genomic hybridization in archival paraffin-embedded tumor samples. Am J Pathol 1994, $145: 1301-1308$.

John HA, Birnstiel ML, Jones KW: RNA-DNA hybrids at the cytological level. Nature 1969, 223:582-587.

Joos S, Fink TM, Ratsch A, Lichter P: Mapping and chromosome analysis: the potential of fluorescence in situ hybridization. J Biotechnol 1994, 35:135-153.

Kallioniemi A, Kallioniemi OP, Sudar D, Rutovit D, Gray J, Waldman F, Pinkel D: Comparative genomic hybridization for molecular cytogenetic analysis of solid tumors. Science 1992, 258:818-821.

Kallioniemi OP, Kallioniemi A, Sudar D, Rutovitz D, Gray JW, Waldman F, Pinkel D: Comparative genomic hybridization: a rapid new method for detecting and mapping DNA amplification in tumors. Semin Cancer Biol 1993, 4:41-46.

Kallioniemi OP, Kallioniemi A, Piper J, Isola J, Waldman FM, Gray JW, Pinkel D: Optimizing comparative genomic hybridization for analysis of DNA sequence copy number changes in solid tumors. Genes Chromosomes Cancer 1994, 10:231-243.

Kan-Mitchell J, Mitchell MS, Rao N, Liggett PE: Characterization of uveal melanoma cell lines that grow as xenografts in rabbit eyes. Invest Ophthalmol Vis Sci 1989, 30:829-834. 
Kincaid MC: Uveal Melanoma. Cancer Control 1998, 5:299-309.

Langer PR, Waldrop AA, Ward DC: Enzymatic synthesis of biotin-labeled polynucleotides: novel nucleic acid affinity probes. Proc Natl Acad Sci USA 1981, 78:6633-6637.

Larouche N, Larouche K, Beliveau A, Leclerc S, Salesse C, Pelletier G, Guerin SL: Transcriptional regulation of the alpha 4 integrin subunit gene in the metastatic spread of uveal melanoma. Anticancer Res 1998, 18:3539-3547.

Lawrence JB, Villnave CA, Singer RH: Sensitive, high-resolution chromatin and chromosome mapping in situ: presence and orientation of two closely integrated copies of EBV in a lymphoma line. Cell 1988, 52:51-61.

Lee C, Gisselsson D, Jin C, Nordgren A, Ferguson DO, Blennow E, Fletcher JA, Morton CC: Limitations of chromosome classification by multicolor karyotyping. Am J Hum Genet 2001, 68:1043-1047.

Lichter P, Cremer T, Borden J, Manuelidis L, Ward DC: Delineation of individual human chromosomes in metaphase and interphase cells by in situ suppression hybridization using recombinant DNA libraries. Hum Genet 1988a, 80:224-234.

Lichter P, Cremer T, Tang CJ, Watkins PC, Manuelidis L, Ward DC: Rapid detection of human chromosome 21 aberrations by in situ hybridization. Proc Natl Acad Sci USA 1988b, 85:9664-9668.

Logani S, Cho AS, Ali BH, Withers HR, McBride WH, Kozlov KL, Hall MO, Lee DA, Straatsma BR: Single-dose compared with fractionated-dose radiation of the OM431 choroidal melanoma cell line. Am J Ophthalmol 1995a, 120:506-510.

Logani S, Cho AS, Su LD, Withers HR, McBride WH, Hall MO, Lee DA, Milani JK, Straatsma BR: Effects of gamma radiation on the OM431 human ocular melanoma cell line. Exp Eye Res 1995b, 60:603605.

Luke S and Shepelsky M: FISH: recent advances and diagnostic aspects. Cell Vis 1998, 5:49-53.

Lundsteen C, Maahr J, Christensen B, Bryndorf T, Bentz M, Lichter P, Gerdes T: Image analysis in comparative genomic hybridization. Cytometry 1995, 19:42-50. 
Luyten GP, Naus NC, Mooy CM, Hagemeijer A, Kan-Mitchell J, Van Drunen E, Vuzevski V, De Jong PT, Luider TM: Establishment and characterization of primary and metastatic uveal melanoma cell lines. Int J Cancer 1996, 66:380-387.

Luyten GP, van der Spek CW, Brand I, Sintnicolaas K, de Waard-Siebinga I, Jager MJ, de Jong PT, Schrier PI, Luider TM: Expression of MAGE, gp100 and tyrosinase genes in uveal melanoma cell lines. Melanoma Res 1998, 8:11-16.

Lynch HT, Anderson DE, Krush AJ: Heredity and intraocular malignant melanoma. Study of two families and review of forty-five cases. Cancer 1968, 21:119-125.

Macville M, Veldman T, Padilla-Nash H, Wangsa D, O’Brien P, Schröck E, Ried T: Spectral karyotyping, a 24-colour FISH technique for the analysis of chromosomal rearrangements. Histochem Cell Bio 1997, 108:299-305.

Macville M, Schröck E, Padilla-Nash H, Keck C, Ghadimi BM, Zimonjic D, Popescu N, Ried T: Comprehensive and definitive molecular cytogenetic characterization of HeLa cells by spectral karyotyping. Cancer Res 1999, 59:141-150.

McLean IW, Foster WD, Zimmerman LE: Prognostic factors in small malignant melanomas of choroid and ciliary body. Arch Ophthalmol 1977, 95:48-58.

McLean IW, Foster WD, Zimmerman LE, Gamel JW: Modifications of Callender's classification of uveal melanoma at the Armed Forces Institute of Pathology. Am J Ophthalmol 1983, 96:502-509.

McLean IW: The biology of haematogenous metastasis in human uveal malignant melanoma. Virchows Arch A Pathol Anat Histopathol 1993, 422:433-437.

McLean, IW: Prognostic Features of Uveal Malignant Melanoma. Ophthalmol Clin North Am 1995, 8:143153.

McLean, W and Gamel JW: Uveal (intraocular) melanoma. Cancer J 1996, 9:20-25.

McNamara M, Felix C, Davison EV, Fenton M, Kennedy SM: Assessment of chromosome 3 copy number in ocular melanoma using fluorescence in situ hybridization. Cancer Genet Cytogenet 1997, 98:4-8. 
McNicol AM and Farquharson MA: In situ hybridization and its diagnostic applications in pathology. J Pathol 1997 Jul, 182:250-261.

Merbs SL and Sidransky D: Analysis of p16 (CDKN2/MTS-1/INK4A) alterations in primary sporadic uveal melanoma. Invest Ophthalmol Vis Sci 1999, 40:779-783.

Myatt N, Aristodemou P, Neale MH, Foss AJ, Hungerford JL, Bhattacharya S, A Cree I: Abnormalities of the transforming growth factor-beta pathway in ocular melanoma. J Pathol 2000, 192:511-518.

Naus NC, van Drunen E, de Klein A, Luyten GP, Paridaens DA, Alers JC, Ksander BR, Beverloo HB, Slater RM: Characterization of complex chromosomal abnormalities in uveal melanoma by fluorescence in situ hybridization, spectral karyotyping, and comparative genomic hybridization. Genes Chromosomes Cancer 2001, 30:267-273.

Naus NC, Verhoeven AC, van Drunen E, Slater R, Mooy CM, Paridaens DA, Luyten GP, de Klein A: Detection of genetic prognostic markers in uveal melanoma biopsies using fluorescence in situ hybridization. Clin Cancer Res 2002, 8:534-539.

Nederlof PM, Robinson D, Abuknesha R, Wiegant J, Hopman AH, Tanke HJ, Raap AK: Three-color fluorescence in situ hybridization for the simultaneous detection of multiple nucleic acid sequences. Cytometry 1989, 10:20-27.

Nederlof PM, van der Flier S, Wiegant J, Raap AK, Tanke HJ, Ploem JS, van der Ploeg M: Multiple fluorescence in situ hybridization. Cytometry 1990, 11:126-131.

Online Mendelian Inheritance in Man, OMIM TM. McKusick-Nathans Institute for Genetic Medicine, Johns Hopkins University (Baltimore, MD) and National Center for Biotechnology Information, National Library of Medicine (Bethesda, MD), 2000. World Wide Web URL: http://www.ncbi.nlm.nih.gov/omim.

Parada LA, Maranon A, Hallen M, Tranberg KG, Stenram U, Bardi G, Johansson B: Cytogenetic analyses of secondary liver tumors reveal significant differences in genomic imbalances between primary and metastatic colon carcinomas. Clin Exp Metastasis 1999, 17:471-479.

Parrella P, Sidransky D, Merbs SL: Allelotype of posterior uveal melanoma: implications for a bifurcated tumor progression pathway. Cancer Res 1999, 59:3032-3037. 
Pereira F, Burnier MN Jr, Shibata H, Wang B, Carey W: Cytomorphometric parameters and the metastatic potential of cutaneous and uveal melanoma: a comparison with prognostic factors. Am J Dermatopathol 2001, 23:304-307.

Pinkel D, Straume T, Gray JW: Cytogenetic analysis using quantitative, high-sensitivity, fluorescence hybridization. Proc Natl Acad Sci USA 1986a, 83:2934-2938.

Pinkel D, Gray JW, Trask B, van den Engh G, Fuscoe J, van Dekken H: Cytogenetic analysis by in situ hybridization with fluorescently labeled nucleic acid probes. Cold Spring Harb Symp Quant Biol 1986b, 51 Pt 1:151-157.

Pinkel D, Landegent J, Collins C, Fuscoe J, Segraves R, Lucas J, Gray J: Fluorescence in situ hybridization with human chromosome-specific libraries: detection of trisomy 21 and translocations of chromosome 4. Proc Natl Acad Sci USA 1988, 85:9138-9142.

Piper J, Rutovitz D, Sudar D, Kallioniemi A, Kallioniemi OP, Waldman FM, Gray JW, Pinkel D: Computer image analysis of comparative genomic hybridization. Cytometry 1995, 19:10-26.

Prescher G, Bornfeld N, Becher R: Nonrandom chromosomal abnormalities in primary uveal melanoma. J Natl Cancer Inst 1990, 82:1765-1769.

Prescher G, Bornfeld N, Becher R: Two subclones in a case of uveal melanoma. Relevance of monosomy 3 and multiplication of chromosome 8q. Cancer Genet Cytogenet 1994, 77:144-146.

Prescher G, Bornfeld N, Friedrichs W, Seeber S, Becher R: Cytogenetics of twelve cases of uveal melanoma and patterns of nonrandom anomalies and isochromosome formation. Cancer Genet Cytogenet $1995,80: 40-46$.

Prescher G, Bornfeld N, Hirche H, Horsthemke B, Jockel K, Becher R: Prognostic implications of monosomy 3 in uveal melanoma. Lancet 1996, 347:1222-1225.

Raap T: Cytometry for CGH. Cytometry 1995, 19:1-3.

Raap AK: Advances in fluorescence in situ hybridization. Mutat Res 1998, 400:287-298.

Regan S, Judge HE, Gragoudas ES, Egan KM: Iris color as a prognostic factor in ocular melanoma. Arch Ophthalmol 1999, 117:811-814. 
Ried T, Baldini A, Rand TC, Ward DC: Simultaneous visualization of seven different DNA probes by in situ hybridization using combinatorial fluorescence and digital imaging microscopy. Proc Natl Acad Sci USA 1992a, 89:1388-1392.

Ried T, Landes G, Dackowski W, Klinger K, Ward DC: Multicolor fluorescence in situ hybridization for the simultaneous detection of probe sets for chromosomes 13, 18, 21, X and $\mathrm{Y}$ in uncultured amniotic fluid cells. Hum Mol Genet 1992b, 1:307-313.

Ried T, Liyanage M, du Manoir S, Heselmeyer K, Auer G, Macville M, Schröck E: Tumor cytogenetics revisited: comparative genomic hybridization and spectral karyotyping. J Mol Med 1997, 75:801-814.

Rosenberg C, Schut TB, Mostert MC, Tanke HJ, Raap AK, Oosterhuis JW, Looijenga LH: Comparative genomic hybridization in hypotriploid/hyperdiploid tumors. Cytometry 1997, 29:113-121.

Rothmann C, Bar-Am I, Malik Z: Spectral imaging for quantitative histology and cytogenetics. Histol Histopathol 1998, 13:921-926.

Rudkin GT and Stollar BD: High resolution detection of DNA-RNA hybrids in situ by indirect immunofluorescence. Nature 1977, 265:472-473.

Schröck E, du Manoir S, Veldman T, Schoell B, Wienberg J, Ferguson-Smith MA, Ning Y, Ledbetter DH, Bar-Am I, Soenksen D, Garini Y, Ried T: Multicolor spectral karyotyping of human chromosomes. Science 1996, 273:494-497.

Schröck E, Veldman T, Padilla-Nash H, Ning Y, Spurbeck J, Jalal S, Shaffer LG, Papenhausen P, Kozma C, Phelan MC, Kjeldsen E, Schonberg SA, O'Brien P, Biesecker L, du Manoir S, Ried T: Spectral karyotyping refines cytogenetic diagnostics of constitutional chromosomal abnormalities. Hum Genet 1997, 101:255-262.

Schröck E and Padilla-Nash H: Spectral karyotyping and multicolor fluorescence in situ hybridization reveal new tumor-specific chromosomal aberrations. Semin Hematol 2000, 37:334-347.

Singh AD, Boghosian-Sell L, Wary KK, Shields CL, De Potter P, Donoso LA, Shields JA, Cannizzaro LA: Cytogenetic findings in primary uveal melanoma. Cancer Genet Cytogenet 1994, 72:109-115.

Singh AD, Wang MX, Donoso LA, Shields CL, De Potter P, Shields JA: Genetic aspects of uveal melanoma: a brief review. Semin Oncol 1996a, 23:768-772. 
Singh AD, Donoso LA, Jackson L, Shields CL, De Potter P, Shields JA: Familial uveal melanoma: absence of constitutional cytogenetic abnormalities in 14 cases. Arch Ophthalmol 1996b, 114:502-503.

Singh AD, Shields CL, Shields JA: Prognostic factors in uveal melanoma. Melanoma Res 2001, 11:255263.

Sisley 1990 Sisley K, Rennie IG, Cottam DW, Potter AM, Potter CW, Rees RC: Cytogenetic findings in six posterior uveal melanomas: involvement of chromosomes 3, 6, and 8. Genes Chromosomes Cancer 1990, 2:205-209.

Sisley K, Cottam DW, Rennie IG, Parsons MA, Potter AM, Potter CW, Rees RC: Non-random abnormalities of chromosomes 3, 6, and 8 associated with posterior uveal melanoma. Genes Chromosomes Cancer 1992, 5:197-200.

Sisley K, Curtis D, Rennie IG, Rees RC: Loss of heterozygosity of the thyroid hormone receptor B in posterior uveal melanoma. Melanoma Res 1993, 3:457-461.

Sisley K, Rennie IG, Parsons MA, Jacques R, Hammond DW, Bell SM, Potter AM, Rees RC: Abnormalities of chromosomes 3 and 8 in posterior uveal melanoma correlate with prognosis. Genes Chromosomes Cancer 1997, 19:22-28.

Sisley K, Parsons MA, Garnham J, Potter AM, Curtis D, Rees RC, Rennie IG: Association of specific chromosome alterations with tumour phenotype in posterior uveal melanoma. Br J Cancer 2000, 82:330338.

Speicher MR, du Manoir S, Schröck E, Holtgreve-Grez H, Schoell B, Lengauer C, Cremer T, Ried T: Molecular cytogenetic analysis of formalin-fixed, paraffin-embedded solid tumors by comparative genomic hybridization after universal DNA-amplification. Hum Mol Genet 1993, 2:1907-1914.

Speicher MR, Prescher G, du Manoir S, Jauch A, Horsthemke B, Bornfeld N, Becher R, Cremer T: Chromosomal gains and losses in uveal melanomas detected by comparative genomic hybridization. Cancer Res 1994, 54:3817-3823.

Speicher MR, Gwyn Ballard S, Ward DC: Karyotyping human chromosomes by combinatorial multifluor FISH. Nat Genet 1996, 12:368-375. 
Telenius H, Pelmear AH, Tunnacliffe A, Carter NP, Behmel A, Ferguson-Smith MA, Nordenskjold M, Pfragner R, Ponder BA: Cytogenetic analysis by chromosome painting using DOP-PCR amplified flowsorted chromosomes. Genes Chromosomes Cancer 1992a, 4:257-263.

Telenius H, Carter NP, Bebb CE, Nordenskjold M, Ponder BA, Tunnacliffe A: Degenerate oligonucleotideprimed PCR: general amplification of target DNA by a single degenerate primer. Genomics 1992b, 13:718725 .

Thompson CT and Gray JW: Cytogenetic profiling using fluorescence in situ hybridization (FISH) and comparative genomic hybridization (CGH). J Cell Biochem Suppl 1993, 17G:139-143.

Tjio JH and Levan A: The chromosome number of man. Hereditas 1956, 42:1-6.

Tonon G, Roschke A, Stover K, Shou Y, Kuehl WM, Kirsch IR: Spectral karyotyping combined with locus-specific FISH simultaneously defines genes and chromosomes involved in chromosomal translocations. Genes Chromosomes Cancer 2000, 27:418-423.

Trask BJ: Fluorescence in situ hybridization: applications in cytogenetics and gene mapping. Trends Genet 1991, 7:149-154.

Tschentscher F, Prescher G, Horsman DE, White VA, Rieder H, Anastassiou G, Schilling H, Bornfeld N, Bartz-Schmidt KU, Horsthemke B, Lohmann DR, Zeschnigk M: Partial deletions of the long and short arm of chromosome 3 point to two tumor suppressor genes in uveal melanoma. Cancer Res 2001, 61:34393442 .

Tucker JD, Lee DA, Ramsey MJ, Briner J, Olsen L, Moore DH $2^{\text {nd }}$ : On the frequency of chromosome exchanges in a control population measured by chromosome painting. Mutat Res 1994, 313:193-202.

Tucker MA, Shields JA, Hartge P, Augsburger J, Hoover RN, Fraumeni JF Jr: Sunlight exposure as risk factor for intraocular malignant melanoma. N Engl J Med 1985, 313:789-792.

University of Michigan Health System, “DNA Denaturation/Renaturation.” 1998, World Wide Web URL: http://www.seqcore.brcf.med.umich.edu/doc/educ/dnapr/pg2.html.

Veldman T, Vignon C, Schröck E, Rowley JD, Ried T: Hidden chromosome abnormalities in haematological malignancies detected by multicolour spectral karyotyping. Nat Genet 1997, 15:406-410. 
Watson JD and Crick FH: Molecular structure of nucleic acids: a structure for deoxyribose nucleic acid. Nature 1953, 4356:737-738.

White VA, McNeil BK, Thiberville L, Horsman DE: Acquired homozygosity (isodisomy) of chromosome 3 during clonal evolution of a uveal melanoma: association with morphologic heterogeneity. Genes Chromosomes Cancer 1996, 15:138-143.

White VA, McNeil BK, Horsman DE: Acquired homozygosity (isodisomy) of chromosome 3 in uveal melanoma. Cancer Genet Cytogenet 1998a, 102:40-45.

White VA, Chambers JD, Courtright PD, Chang WY, Horsman DE: Correlation of cytogenetic abnormalities with the outcome of patients with uveal melanoma. Cancer 1998b, 83:354-359.

Wiltshire RN, Elner VM, Dennis T, Vine AK, Trent JM: Cytogenetic analysis of posterior uveal melanoma. Cancer Genet Cytogenet 1993, 66:47-53. 
APPENDIX A

\section{BUFFERS, REAGENTS AND SOLUTIONS}




\section{Cell Culture}

\section{$\underline{\text { RPMI } 1640}$}

1 package powdered RPMI 1640 media (Invitrogen Life Technologies, Carlsbad, CA)

$2 \mathrm{~g} \mathrm{NaHCO}_{3}$

$1000 \mathrm{ml}$ sterile or autoclaved $\mathrm{dH}_{2} \mathrm{O}$

Add powdered media and approximately $900 \mathrm{ml}$ sterile/autoclaved $\mathrm{dH}_{2} \mathrm{O}$ to $1000 \mathrm{ml}$ volumetric flask. Add $2 \mathrm{~g} \mathrm{NaHCO}_{3}$, and add a stir bar. When dissolved, $\mathrm{pH}$ to 0.2-0.3 below final desired $\mathrm{pH}$ (7.0-7.4). Remove stir bar, and add remainder of $\mathrm{dH}_{2} \mathrm{O}$ to reach $1000 \mathrm{ml}$. Using suction filtration, divide flask into two $500 \mathrm{ml}$ sterile bottles. Incomplete media can be stored at $-20^{\circ} \mathrm{C}$ until needed.

To complete media (each $500 \mathrm{ml}$ bottle):

50ml FBS (Sigma Co., St. Louis, MO)

$5 \mathrm{ml}$ NEAA (Sigma Co., St. Louis, MO)

$5 \mathrm{ml}$ HEPES Buffer (Sigma Co., St. Louis, MO)

$0.5 \mathrm{ml}$ gentamicin (Invitrogen Life Technologies, Carlsbad, CA)

0.5-1 ml Fungizone (Invitrogen Life Technologies, Carlsbad, CA)

$1-2 \mathrm{ml}$ penicillin/streptomycin $(100 \mathrm{U} / \mathrm{ml}$ and $100 \mu \mathrm{g} / \mathrm{ml}$, respectively) (Invitrogen Life Technologies, Carlsbad, CA)

Store completed media at $4^{\circ} \mathrm{C}$ in the dark.

\section{DMEM}

1 bottle powdered DMEM media (Sigma Co., St. Louis, MO)

$3.7 \mathrm{~g} \mathrm{NaHCO}_{3}$

$1000 \mathrm{ml}$ sterile or autoclaved $\mathrm{dH}_{2} \mathrm{O}$

Add powdered media and approximately $900 \mathrm{ml}$ sterile/autoclaved $\mathrm{dH}_{2} \mathrm{O}$ to $1000 \mathrm{ml}$ volumetric flask. Add $3.7 \mathrm{~g} \mathrm{NaHCO}_{3}$, and add a stir bar. When dissolved, $\mathrm{pH}$ to 0.2-0.3 below final desired $\mathrm{pH}(7.0 \pm 0.3)$. Remove stir bar, and add remainder of $\mathrm{dH}_{2} \mathrm{O}$ to reach $1000 \mathrm{ml}$. Using suction filtration, divide flask into two $500 \mathrm{ml}$ sterile bottles. Incomplete media can be stored at $-20^{\circ} \mathrm{C}$ until needed.

To complete media (each $500 \mathrm{ml}$ bottle):

$50 \mathrm{ml}$ FBS

$5 \mathrm{ml}$ NEAA

$5 \mathrm{ml}$ HEPES Buffer

$0.5 \mathrm{ml}$ gentamycin

0.5-1ml Fungizone

$1-2 \mathrm{ml}$ penicillin/streptomycin

Store completed media at $4^{\circ} \mathrm{C}$ in the dark.

\section{$\underline{\text { Ham's F-12 }}$}

1 package powdered Ham's F-12 media (Invitrogen Life Technologies, Carlsbad, CA)

$1.176 \mathrm{~g} \mathrm{NaHCO}_{3}$

$1000 \mathrm{ml}$ sterile or autoclaved $\mathrm{dH}_{2} \mathrm{O}$

Add powdered media and approximately $900 \mathrm{ml}$ sterile/autoclaved $\mathrm{dH}_{2} \mathrm{O}$ to $1000 \mathrm{ml}$ volumetric flask. Add $1.176 \mathrm{~g} \mathrm{NaHCO}_{3}$, and add a stir bar. When dissolved, $\mathrm{pH}$ to 0.2-0.3 below final desired $\mathrm{pH}$ (7.6-7.9). Remove stir bar, and add remainder of $\mathrm{dH}_{2} \mathrm{O}$ to reach $1000 \mathrm{ml}$. Using suction filtration, divide flask into two $500 \mathrm{ml}$ sterile bottles. Incomplete media can be stored at $-20^{\circ} \mathrm{C}$ until needed. 
To complete media (each $500 \mathrm{ml}$ bottle):

$50 \mathrm{ml}$ FBS

$5 \mathrm{ml} \mathrm{NEAA}$

$5 \mathrm{ml}$ HEPES Buffer

$0.5 \mathrm{ml}$ gentamycin

0.5-1 $\mathrm{ml}$ Fungizone

$1-2 \mathrm{ml}$ penicillin/streptomycin

Store completed media at $4^{\circ} \mathrm{C}$ in the dark.

\section{Metaphase Chromosome Preparation}

Carnoy's Fixative

$75 \mathrm{ml}$ absolute methanol, HPLC grade (Fisher Scientific, Inc., Pittsburgh, PA)

$25 \mathrm{ml}$ glacial acetic acid, tracemetal grade (Fisher Scientific, Pittsburgh, PA)

Make 3:1 mixture as needed. Use only when fresh (first day).

\section{$\frac{75 \mathrm{mM} \mathrm{KCl}}{5.6 \mathrm{gCl}}$}

$5.6 \mathrm{~g} \mathrm{KCl}$

$1000 \mathrm{ml} \mathrm{dH_{2 } \mathrm { O }}$

Dissolve $\mathrm{KCl}$ in $\mathrm{dH}_{2} \mathrm{O}$. Store at room temperature.

\section{DNA Isolation}

\section{$100 \mathrm{mM}$ Tris/Cl pH 8.5}

2.21g Tris - $\mathrm{HCl}$ (Sigma Co., St. Louis, MO)

4.36g Trizma Base (Sigma Co., St. Louis, MO)

Add Tris - $\mathrm{HCL}$, Trizma base and $\sim 450 \mathrm{ml} \mathrm{dH}_{2} \mathrm{O}$ to a $500 \mathrm{ml}$ volumetric flask with a magnetic stir bar. After contents are completely dissolved, remove stir bar and fill flask the remainder of the way to the $500 \mathrm{ml}$ mark with $\mathrm{dH}_{2} \mathrm{O}$. Adjust $\mathrm{pH}$ to 8.5 and autoclave. Store at room temperature.

\section{Cell Lysis Buffer}

$20 \mathrm{ml} 100 \mathrm{mM}$ Tris $-\mathrm{HCl} \mathrm{pH} 8.5$

2ml 0.5M EDTA pH 8.0 (Digene, Gaithersburg, MD)

$4 \mathrm{ml} 10 \%$ SDS (Fisher Scientific, Pittsburgh, PA)

$8 \mathrm{ml} 5 \mathrm{M} \mathrm{NaCl}$

$165 \mathrm{ml} \mathrm{dH_{2 } \mathrm { O }}$

Makes $199 \mathrm{ml}$. Store at room temperature.

\section{TE Buffer $\mathrm{pH} 7.0$}

$5 \mathrm{ml} 1 \mathrm{M}$ Tris - $\mathrm{HCl} \mathrm{pH} 7.0$ (Quality Biological, Inc., Gaithersburg, MD)

$1 \mathrm{ml} 0.5 \mathrm{M}$ EDTA $\mathrm{pH} 8.0$

$494 \mathrm{ml} \mathrm{dH_{2 } \mathrm { O }}$

Adjust to $\mathrm{pH} 7.0$ and autoclave. Store at room temperature.

\section{$\underline{\text { RNase A }}$}

100mg RNase A, lyophilized (Roche Molecular Biochemicals, Indianapolis, IN)

$5 \mathrm{ml}$ sterile $\mathrm{H}_{2} \mathrm{O}$ (DNase free, RNase free) 
To dissolve, boil for 15 minutes, and then cool to room temperature. Aliquot $20 \mathrm{mg} / \mathrm{ml}$ solution, and store at $-20^{\circ} \mathrm{C}$. See also "Pretreatment of Slides for CGH, SKY and FISH."

\section{Nick Translation}

\section{NT Buffer}

$500 \mu 1$ 0.5M Tris HCl, pH 8.0 (Quality Biological, Inc., Gaithersburg, MD)

$100 \mu \mathrm{l} 50 \mathrm{mM} \mathrm{MgCl}$

$50 \mu 10.5 \mathrm{mg} / \mathrm{ml}$ BSA (Sigma Co., St. Louis, MO)

$350 \mu$ sterile $\mathrm{H}_{2} \mathrm{O}$ (DNase free, RNase free) (Sigma Co., St. Louis, MO)

Makes $1 \mathrm{ml}$. Aliquot and store at $-20^{\circ} \mathrm{C}$.

DNase I $(1 \mathrm{mg} / \mathrm{ml})$

10mg DNase I (Roche Molecular Biochemicals, Indianapolis, IN)

$1.5 \mathrm{ml} 0.15 \mathrm{M} \mathrm{NaCl}$ (Quality Biological, Inc., Gaithersburg, MD)

$5 \mathrm{ml} \mathrm{50 \%} \mathrm{Glycerol} \mathrm{(Fisher} \mathrm{Scientific,} \mathrm{Pittsburgh,} \mathrm{PA)}$

$10 \mathrm{ml}$ sterile $\mathrm{H}_{2} \mathrm{O}$ (DNase free, RNase free)

Aliquot and store at $-20^{\circ} \mathrm{C}$. Make 1:1000 dilution for use in nick translation reactions.

dNTP Mixture

$5 \mu 1$ each of 100mM dATP, dCTP, and dGTP (Promega, Madison, WI)

$1 \mu 1$ of $100 \mathrm{mM}$ dTTP (Promega, Madison, WI)

$984 \mu \mathrm{l}$ sterile $\mathrm{H}_{2} \mathrm{O}$ (DNase free, RNase free)

Makes $0.5 \mathrm{mM} \mathrm{dATP} / \mathrm{dCTP} / \mathrm{dGTP}$ and $0.05 \mathrm{mM}$ dTTP. Aliquot and store at $-20^{\circ} \mathrm{C}$.

$\underline{0.1 \mathrm{M} \beta \text {-Mercaptoethanol }}$

$34.7 \mu 1$ of $99 \%$ (14.4M) solution (Sigma Co., St. Louis, MO)

Sterile $\mathrm{H}_{2} \mathrm{O}$ (DNase free, RNase free) to make $5 \mathrm{ml}$

Aliquot and store at $-20^{\circ} \mathrm{C}$.

$\underline{50 \mu 1 \text { Nick Translation Reaction }}$

$\mathrm{x} \mu \mathrm{l}(1 \mu \mathrm{g})$ DNA (volume variable)

$5 \mu 1$ 10xNT Buffer

$5 \mu 1$ dNTP mixture

$5 \mu 10.1 \mathrm{M} ß$-Mercaptoethanol

$2 \mu 1$ biotin- or digoxigenin-11-dUTP

33- $\mathrm{x} \mu \mathrm{l}$ sterile $\mathrm{H}_{2} \mathrm{O}$ (DNase free, RNase free)

\section{DOP-PCR}

\section{DOP-PCR Primer}

Resuspend DOP-PCR CGH primer, 5' - GAT CAA GCT NNN NNN ATG TGG - 3' (Integrated DNA Technologies, Inc., Coralville, IA) in $1223.7 \mu \mathrm{l}$ sterile $\mathrm{H}_{2} \mathrm{O}$ (DNase free, RNase free) to make $100 \mathrm{mM}$ primer stock.

Dilute primer stock 1:5 in sterile $\mathrm{H}_{2} \mathrm{O}$ (DNase free, RNase free) to make 20mM primer. 
Biotin- or dig-dNTP Mixture

$3.04 \mu 1 \mathrm{dATP}$

$3.04 \mu 1 \mathrm{dCTP}$

$3.04 \mu 1 \mathrm{dGTP}$

$1.26 \mu 1 \mathrm{dTTP}$

$25 \mu 1$ biotin- or dig-dUTP (Roche Molecular Biochemicals, Indianapolis, IN)

$117 \mu$ l sterile $\mathrm{H}_{2} \mathrm{O}$ (DNase free, RNase free)

Makes $152 \mu \mathrm{l}$ dNTP mix. Make fresh as needed.

DOP-PCR Polymerase

250U AmpliTaq Gold Polymerase stock, 5U/ $\mu 1$ (Roche Applied Biosystems, Foster City, CA)

$50 \mu 1$ sterile $\mathrm{H} 2 \mathrm{O}$ (DNase free, RNase free)

Make 1:1 dilution $(2.5 \mathrm{U} / \mu \mathrm{l})$ of AmpliTaq Gold Polymerase before use, by adding $50 \mu \mathrm{l}$ sterile $\mathrm{H} 2 \mathrm{O}$ to $50 \mu \mathrm{l}$ AmpliTaq Gold stock.

\section{DOP-PCR Master Mix (MM)}

10 $\mu 1$ 10xBuffer II (Roche Applied Biosystems, Foster City, CA)

$10 \mu 120 \mathrm{mM}$ DOP Primer (Integrated DNA Technologies, Inc., Coralville, IA)

$24 \mu 125 \mathrm{mM} \mathrm{MgCl} 2$ (Roche Applied Biosystems, Foster City, CA)

$5 \mu$ l biotin- or dig-dUTP mixture

$39 \mu \mathrm{l}$ sterile $\mathrm{H}_{2} \mathrm{O}$ (DNase free, RNase free)

$2 \mu 1$ AmpliTaq Gold Polymerase

Makes $90 \mu \mathrm{l} \mathrm{MM}$, which is enough for a single DOP-PCR reaction. Scale as needed, allowing for a single extra reaction in case of error. Make fresh as needed and keep on ice.

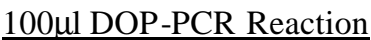

$10 \mu 1$ DNA

$90 \mu 1$ DOP-PCR MM

Reaction Conditions:

$95^{\circ} \mathrm{C}$ for 10 minutes, initial soak

15 cycles:

$94^{\circ} \mathrm{C}$ for 1 minute

$30^{\circ} \mathrm{C}$ for 1 minute

$72^{\circ} \mathrm{C}$ for 1 minute

60 cycles (with a 1 second per cycle addition to each step in the 60 cycles):

$94^{\circ} \mathrm{C}$ for 30 seconds

$52^{\circ} \mathrm{C}$ for 30 seconds

$72^{\circ} \mathrm{C}$ for 1 minute

Final extension of $72^{\circ} \mathrm{C}$ for 10 minutes

Hold at $4^{\circ} \mathrm{C}$.

\section{Electrophoresis}

\section{$1.7 \%$ Agarose Gel}

$0.85 \mathrm{~g}$ low melting point agarose powder (Invitrogen Life Technologies, Carlsbad, CA)

50ml 1x TBE buffer (Invitrogen Life Technologies, Carlsbad, CA)

Microwave (boil) until dissolved. Cool to $\sim 65^{\circ} \mathrm{C}$ in a water bath and mix in $2.5 \mathrm{ml}$ of a $10 \mathrm{mg} / \mathrm{ml}$ ethidium bromide solution. Pour gel or store at $4{ }^{\circ} \mathrm{C}$ in the dark. 
Gel-Loading Buffer III (6x)

$0.025 \mathrm{~g}$ bromophenol blue (Sigma Co., St. Louis, MO)

$0.025 \mathrm{~g}$ xylene cyanol (Sigma Co., St. Louis, MO)

$3 \mathrm{ml}$ glycerol

$6.95 \mathrm{ml} \mathrm{dH}_{2} \mathrm{O}$

Final solution is $0.25 \%$ bromophenol blue, $0.25 \%$ xylene cyanol, and $30 \%$ glycerol in $\mathrm{dH}_{2} \mathrm{O}$. Store at $4^{\circ} \mathrm{C}$.

\title{
Pretreatment for CGH, SKY and FISH
}

\author{
$\underline{2 x S S C}$ \\ 10ml 20xSSC (Fisher Scientific, Pittsburgh, PA) \\ $90 \mathrm{ml} \mathrm{dH_{2 } \mathrm { O }}$
}

Store at room temperature.

$\underline{1 \times P B S}$

10ml 10xPBS (w/o $\mathrm{MgCl}_{2}$ or $\mathrm{CaCl}_{2}$ ) (Invitrogen Life Technologies, Carlsbad, CA)

$90 \mathrm{ml} \mathrm{dH_{2 } \mathrm { O }}$

Store at room temperature.

\section{$\underline{1 \mathrm{M} \mathrm{MgCl}} \underline{2}_{2}$}

203.3g MgCl${ }_{2} \bullet 6 \mathrm{H}_{2} 0$ (Fisher Scientific, Pittsburgh, PA)

$1000 \mathrm{ml} \mathrm{dH_{2 } \mathrm { O }}$

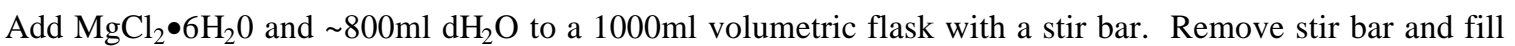
flask to $1000 \mathrm{ml}$. Aliquot and autoclave. Store at room temperature.

$1 \times \mathrm{PBS} / \mathrm{MgCl}_{2}$

$50 \mathrm{ml} 1 \mathrm{M} \mathrm{MgCl}{ }_{2}$

950ml 1xPBS (w/o $\mathrm{MgCl}_{2}$ or $\mathrm{CaCl}_{2}$ )

Store at room temperature.

\section{$\underline{\text { RNase A }}$}

100mg RNase A, lyophilized (Roche Molecular Biochemicals, Indianapolis, IN)

$5 \mathrm{ml}$ sterile $\mathrm{H}_{2} \mathrm{O}$ (DNase free, RNase free)

To dissolve, boil for 15 minutes, and then cool to room temperature. Aliquot $20 \mathrm{mg} / \mathrm{ml}$ solution, and store at $-20^{\circ} \mathrm{C}$. Make 1:200 dilution in $2 \mathrm{xSSC}$ for slide pretreatment.

\section{Pepsin stock solution}

100mg pepsin (Sigma Co., St. Louis, MO)

$1 \mathrm{ml}$ sterile $\mathrm{H}_{2} \mathrm{O}$ (DNase free, RNase free)

Dissolve, keep on ice. Make $50 \mu 1$ aliquots of $100 \mathrm{mg} / \mathrm{ml}$ stock solution, and store at $-20^{\circ} \mathrm{C}$.

\section{$\underline{0.01 \mathrm{M} \mathrm{HCl}}$}

$1 \mathrm{ml} 1 \mathrm{M} \mathrm{HCl}$ (Fisher Scientific, Pittsburgh, PA)

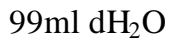

Store at room temperature. 
$\underline{\text { Pepsin/HCl }}$

$15 \mu 1$ pepsin stock solution

$100 \mathrm{ml} 0.01 \mathrm{M} \mathrm{HCl}$

Make fresh as needed with $\mathrm{HCl}$ pre-warmed to $37^{\circ} \mathrm{C}$.

$1 \%$ Formaldehyde in $1 \times \mathrm{PBS} / \mathrm{MgCl}_{2}$

$2.7 \mathrm{ml}$ of $37 \%$ Formaldehyde solution (Fisher Scientific, Pittsburgh, PA)

$100 \mathrm{ml} 1 \times \mathrm{PBS} / \mathrm{MgCl}_{2}$

Store at room temperature.

\title{
Hybridization for CGH, SKY and FISH
}

\section{$\underline{\text { Master Mix (MM) }}$}

$1 \mathrm{~g}$ dextran sulfate sodium salt (Biotech grade) (Fisher Scientific, Pittsburgh, PA)

$5 \mathrm{ml} 2 \mathrm{xSSC}$

Dissolve dextran sulfate in $2 \mathrm{xSSC}$ at $70^{\circ} \mathrm{C}$ for several hours. Once dissolved, cool to room temperature and adjust to $\mathrm{pH}$ 7.0. Aliquot, store at $-20^{\circ} \mathrm{C}$.

70\% FA/SSC for CGH slide denaturation

$7 \mathrm{ml}$ formamide, molecular grade (Fisher Scientific, Pittsburgh, PA)

$1 \mathrm{ml} 20 \mathrm{xSSC}$

$2 \mathrm{ml}$ sterile $\mathrm{H}_{2} \mathrm{O}$ (DNase free, RNase free)

Adjust to $\mathrm{pH}$ 7.2-7.3. Store at $-20^{\circ} \mathrm{C}$.

70\% FA/SSC for FISH slide denaturation

$35 \mathrm{ml}$ formamide, molecular grade

$5 \mathrm{ml} 20 \mathrm{xSSC}$

$10 \mathrm{ml}$ sterile $\mathrm{H}_{2} \mathrm{O}$ (DNase free, RNase free)

Adjust to $\mathrm{pH}$ 7.0. Store at $4^{\circ} \mathrm{C}$ for up to 3 weeks.

Probe Precipitation for CGH

$30 \mu 1$ each (tumor and reference) DOP-PCR product

$60 \mu 1$ human Cot-1 DNA (Invitrogen Life Technologies, Carlsbad, CA)

$1 \mu 1$ salmon testes DNA (Sigma Co., St. Louis, MO)

$12.1 \mu 13 \mathrm{M}$ sodium acetate

$400 \mu$ labsolute ethanol, ice cold

\section{Detection for CGH, SKY and FISH}

\author{
50\% FA/SSC \\ $30 \mathrm{ml} 20 \mathrm{xSSC}$ \\ $120 \mathrm{ml} \mathrm{dH}_{2} \mathrm{O}$ \\ $150 \mathrm{ml}$ formamide, molecular grade
}

Make one day before needed. Adjust to $\mathrm{pH} \sim 7.25$. 


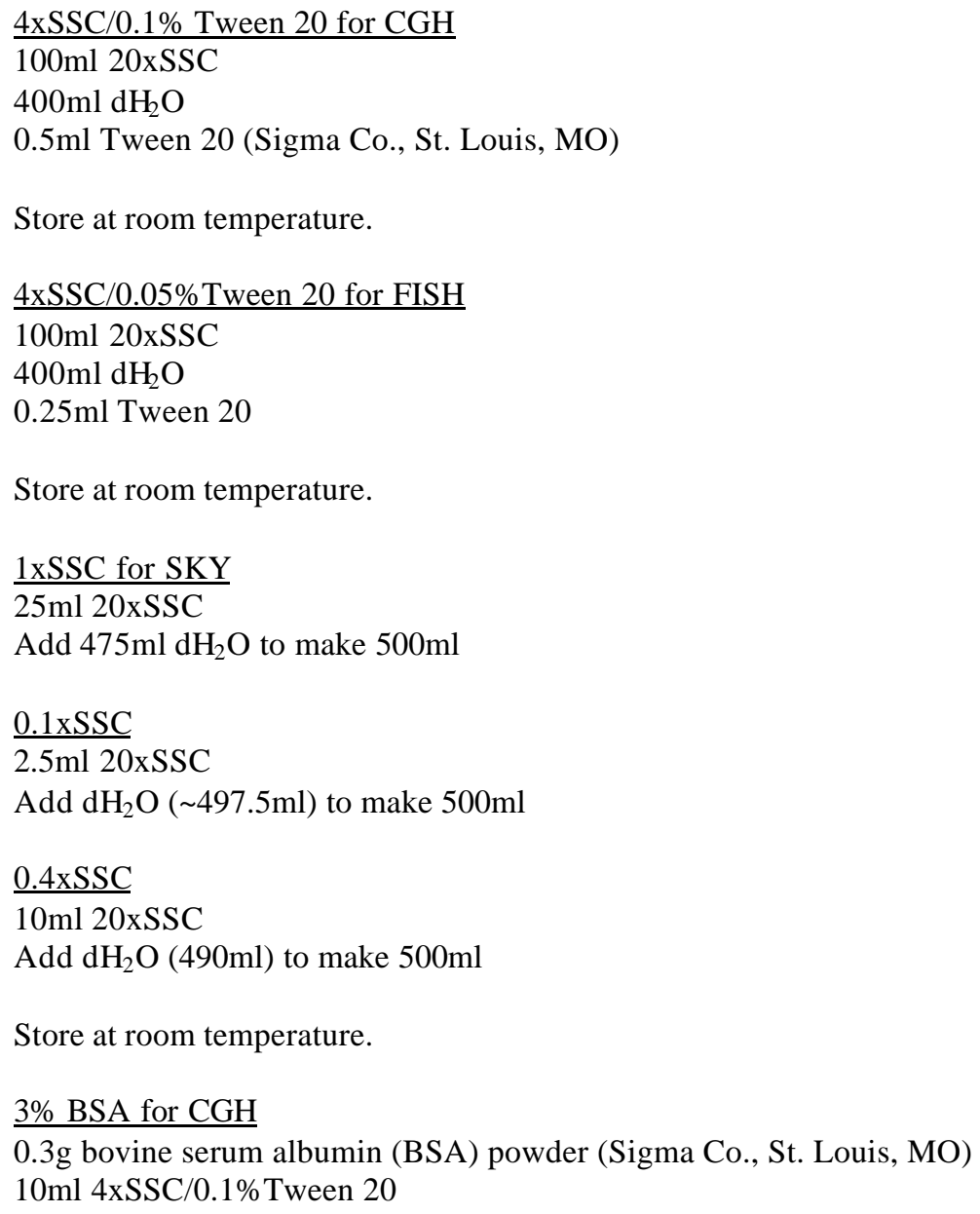

Store at room temperature.

$1 \mathrm{xSSC}$ for SKY

25ml 20xSSC

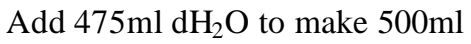

$\underline{0.1 \times S S C}$

$2.5 \mathrm{ml} \mathrm{20xSSC}$

Add $\mathrm{dH}_{2} \mathrm{O}(\sim 497.5 \mathrm{ml})$ to make $500 \mathrm{ml}$

\section{$\underline{0.4 \mathrm{xSSC}}$}

$10 \mathrm{ml} 20 \mathrm{xSSC}$

Add $\mathrm{dH}_{2} \mathrm{O}(490 \mathrm{ml})$ to make $500 \mathrm{ml}$

Store at room temperature.

\section{3\% BSA for $\mathrm{CGH}$}

$0.3 \mathrm{~g}$ bovine serum albumin (BSA) powder (Sigma Co., St. Louis, MO)

$10 \mathrm{ml} 4 \mathrm{xSSC} / 0.1 \%$ Tween 20

Dissolve BSA in $4 \times$ SSC/Tween 20 to make $3 \%$ blocking solution. Store at $4^{\circ} \mathrm{C}$.

$1 \%$ BSA for Antibody Layering in $\mathrm{CGH}$

Dilute 3\% BSA 2:1 in 4xSSC/0.1\% Tween 20

Used in antibody dilution for detection layers in CGH. Store at $4^{\circ} \mathrm{C}$.

\section{$3 \%$ NFDM for FISH}

1 bottle lyophilized NFDM (Sigma Co., St. Louis, MO)

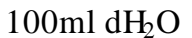

$0.2 \%$ sodium azide

Reconstitute NFDM in $\mathrm{dH}_{2} \mathrm{O}$. Add $200 \mu \mathrm{l}$ sodium azide as a preservative. Makes $3 \%$ blocking solution. Also used in antibody dilution for detection layers in FISH. Store at $4^{\circ} \mathrm{C}$.

\section{$\underline{\text { Antibody Layers for CGH }}$}

1) avidin-FITC (1:200), mouse anti-dig (1:100), in 1\% BSA

2) anti-avidin (1:200), rabbit anti-mouse TRITC (1:200), in 1\% BSA

3) avidin-FITC (1:200), in 1\% BSA

4) goat anti-rabbit TRITC (1:200), in 1\% BSA

$1 \%$ BSA is dilution of 3\% BSA blocking solution for CGH. Prepare $200 \mu$ l of each layer per slide. Make fresh. 
Antibody Layers for FISH

1) avidin-FITC (1:400), avidin-Rhodamine (1:400), in 3\% NFDM

2) anti-avidin (1:100), in 3\% NFDM

3) avidin-FITC (1:400), avidin-Rhodamine (1:400), in 3\% NFDM

$3 \%$ NFDM is also used in blocking layer for FISH. Prepare $200 \mu 1$ of each layer per slide. Make fresh.

$\underline{\text { DAPI }}$

2mg DAPI (Roche Molecular Biochemicals, Indianapolis, IN)

$10 \mathrm{ml} 2 \mathrm{xSSC}$

Dissolve DAPI in $2 \mathrm{xSSC}$ to make $2 \mathrm{mg} / \mathrm{ml}$ DAPI stock solution. Working solution is $80 \mathrm{ng} / \mathrm{ml}$. To make working solution, add $4 \mu 1$ DAPI stock solution to $50 \mathrm{ml} 2 \mathrm{xSSC}$.

$\underline{0.5 \mathrm{M} \text { carbonate buffer }}$

$6.2 \mathrm{~g} \mathrm{Na}_{2} \mathrm{CO}_{3} \cdot \mathrm{H}_{2} \mathrm{O}$ or $5.3 \mathrm{~g} \mathrm{Na}_{2} \mathrm{CO}_{3}$ (Sigma Co., St. Louis, MO)

autoclaved $\mathrm{dH}_{2} \mathrm{O}$ to complete $100 \mathrm{ml}$

Dissolve to make $100 \mathrm{ml}$ of $0.5 \mathrm{M} \mathrm{Na}_{2} \mathrm{CO}_{3}$.

$0.5 \mathrm{M}$ bicarbonate buffer

4.2g $\mathrm{NaHCO}_{3}$ (Sigma Co., St. Louis, MO)

autoclaved $\mathrm{dH}_{2} \mathrm{O}$ to complete $100 \mathrm{ml}$

Dissolve to make $100 \mathrm{ml}$ of $0.5 \mathrm{M} \mathrm{NaHCO}_{3}$.

$\underline{0.5 \mathrm{M} \text { carbonate-bicarbonate buffer }}$

$10 \mathrm{ml} 0.5 \mathrm{M} \mathrm{Na}_{2} \mathrm{CO}_{3}$

$90 \mathrm{ml} 0.5 \mathrm{M} \mathrm{NaHCO}_{3}$

Adjust to $\mathrm{pH}$ 9.0. Store at room temperature.

$\underline{\text { Antifade }}$

100mg phenylenediamine dihydrochloride (Sigma Co., St. Louis, MO)

$10 \mathrm{ml} 1 \mathrm{xPBS}$ (w/o $\mathrm{MgCl}_{2}$ or $\mathrm{CaCl}_{2}$ )

$90 \mathrm{ml}$ glycerol

Dissolve phenylenediamine dihydrochloride in $1 \mathrm{xPBS}$, and sterile filter solution. Adjust $\mathrm{pH}$ to 8.0 with $0.5 \mathrm{M}$ carbonate-bicarbonate buffer $(\mathrm{pH} 9.0)$, and add $90 \mathrm{ml}$ glycerol for a total volume of approximately $110 \mathrm{ml}$. Store at $-20^{\circ} \mathrm{C}$ in the dark (may be useful to wrap bottle in aluminum foil to avoid degradation when using it at room temperature). 
APPENDIX B

MOLECULAR CYTOGENETICS 

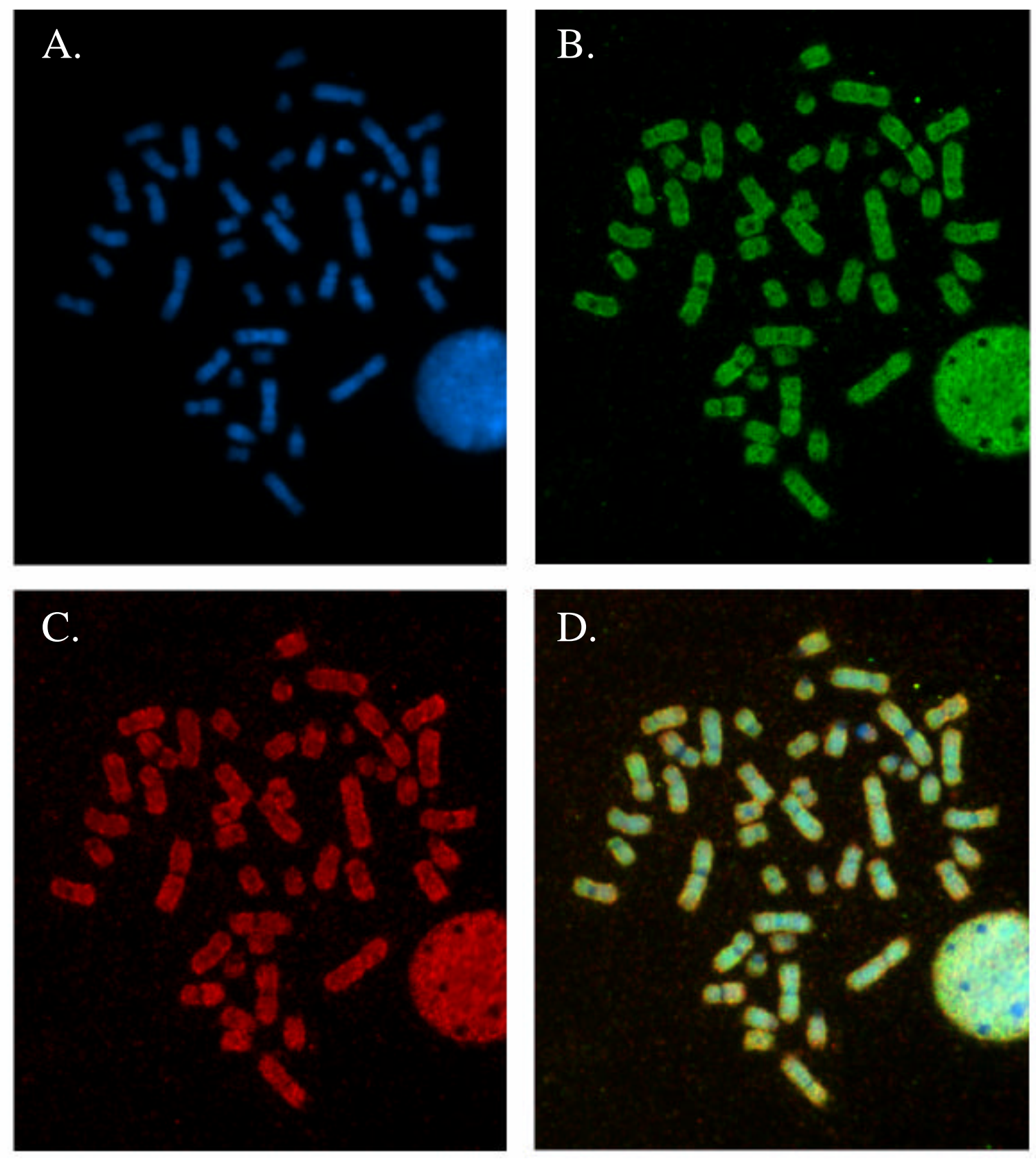

Figure 35. Image Capture for CGH Analysis from an Archival Uveal Melanoma Case. Each of these images is the identical normal male metaphase spread detected with three different fluorescent filters. (A) The metaphase chromosomes counterstained with DAPI. Biotinylated tumor DNA and dig-11-dUTP-labelled normal DNA signals were detected with avidin-conjugated fluorescein isothiocyanate (FITC) and tetramethylrhodamine isothiocyanate (TRITC) conjugated antibodies. (B) The FITC image, and (C) The TRITC image. These three fluorescent images (A, B and C) combine to form the completed CGH image seen in (D). This newly combined image is suitable for CGH analysis; the image is classified and karyotyped, followed by the generation of $\mathrm{CGH}$ profiles to illustrate the amplifications and deletions present within the tumor genome. 

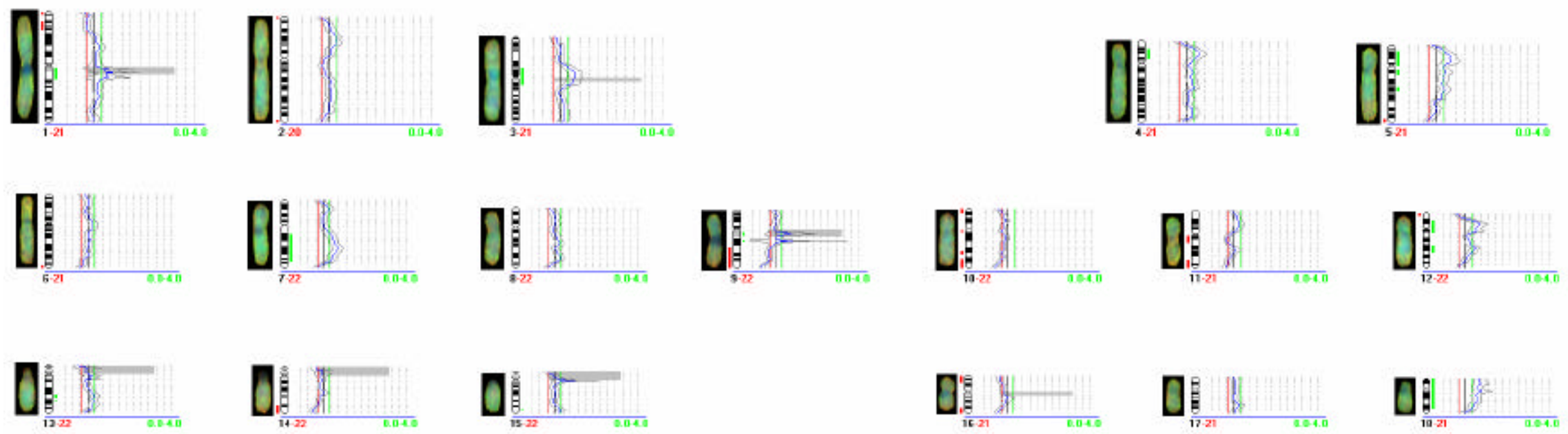

प罚
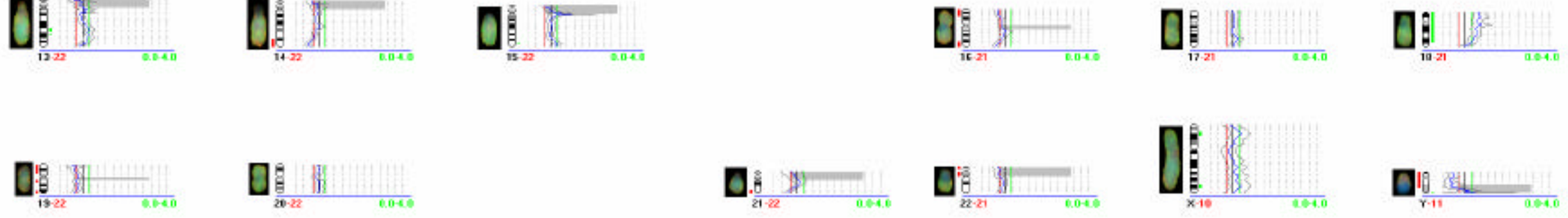

Figure 36. An Example of CGH Profile Data. The Leica image analysis software (Leica Inc., Exton, PA) generates 95\% confidence intervals for mean CGH profile data once each metaphase has been appropriately karyotyped. This profile data is fromcase 39, a male patient. A minimum of ten metaphase spreads were collated to generate the mean data for each of the cases. Using the profiles from each of the spreads that combine to form the mean for each case, the software is able to calculate these confidence intervals for the mean profile data. The color chromosome image to the left of each ideogram (from Fig. 35D) was generated using the combination of all three fluorescent filters seen in Figs. 35A, B, and C. It should be noted that in the case $\mathrm{d}$ ata represented above, the profile data for the chromosomes have relatively narrow confidence intervals, which lends credence to the values of the mean. Simplified, the less variation (width) in the confidence interval, the greater certainty that can be assigned to the relative copy numbers in the data. This kind of data is generated for the mean profile data for the data set of each case during analysis. 

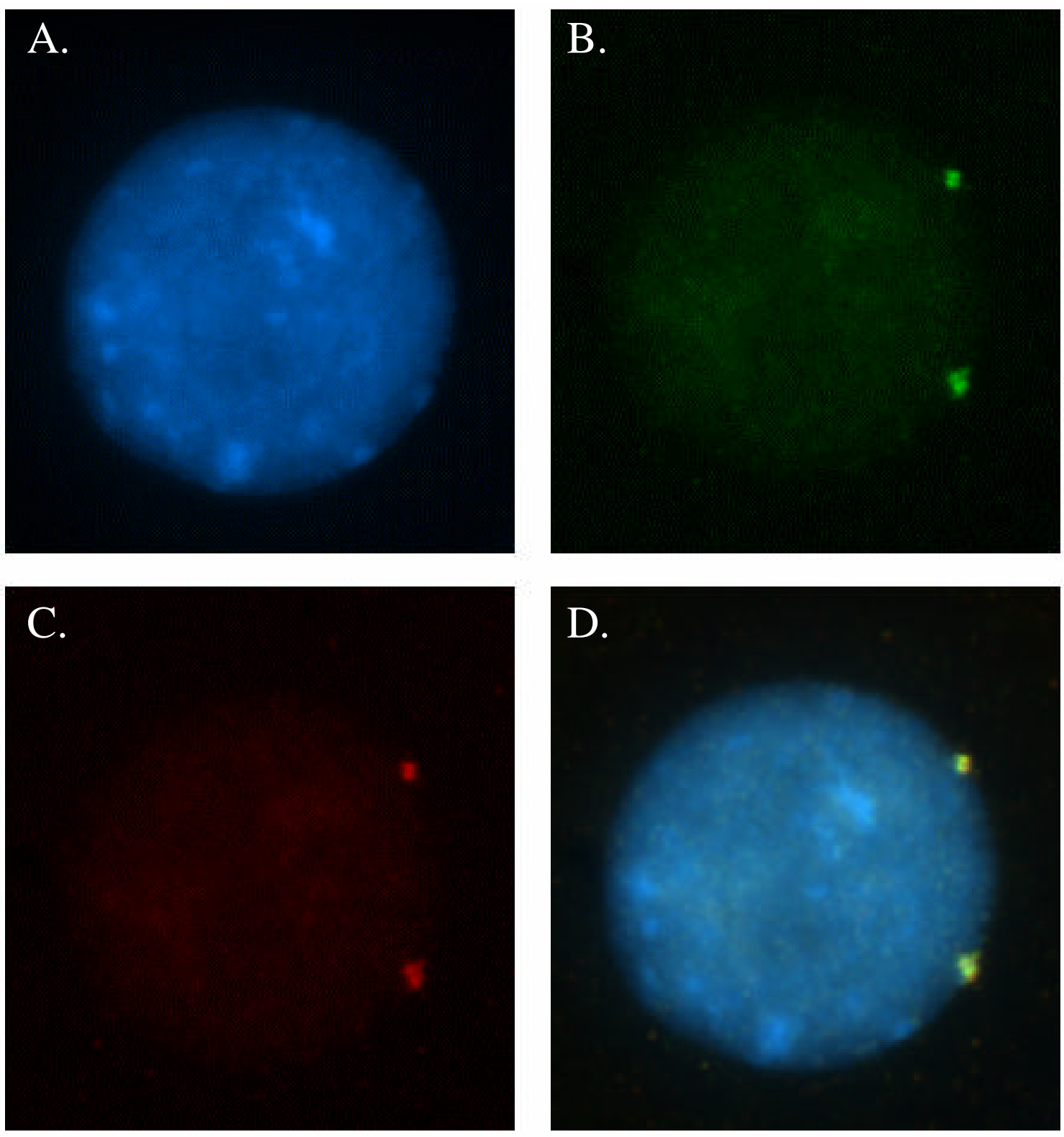

Figure 37. Interphase FISH Control Using Centromere Probe for Chromosome 3. (A) The isolated nucleus is counterstained with DAPI. (B) The FITC and (C) the TRITC images represent the dual-color fluorescence visualization of the centromere probe, ensuring distinction of the signal from the background. (D) The overlay shows 2 yellow signals that confirm the 2 signals visualized are in fact the centromere probe and not background noise. The overlay also confirms that the control nucleus in this image is in fact normal. 

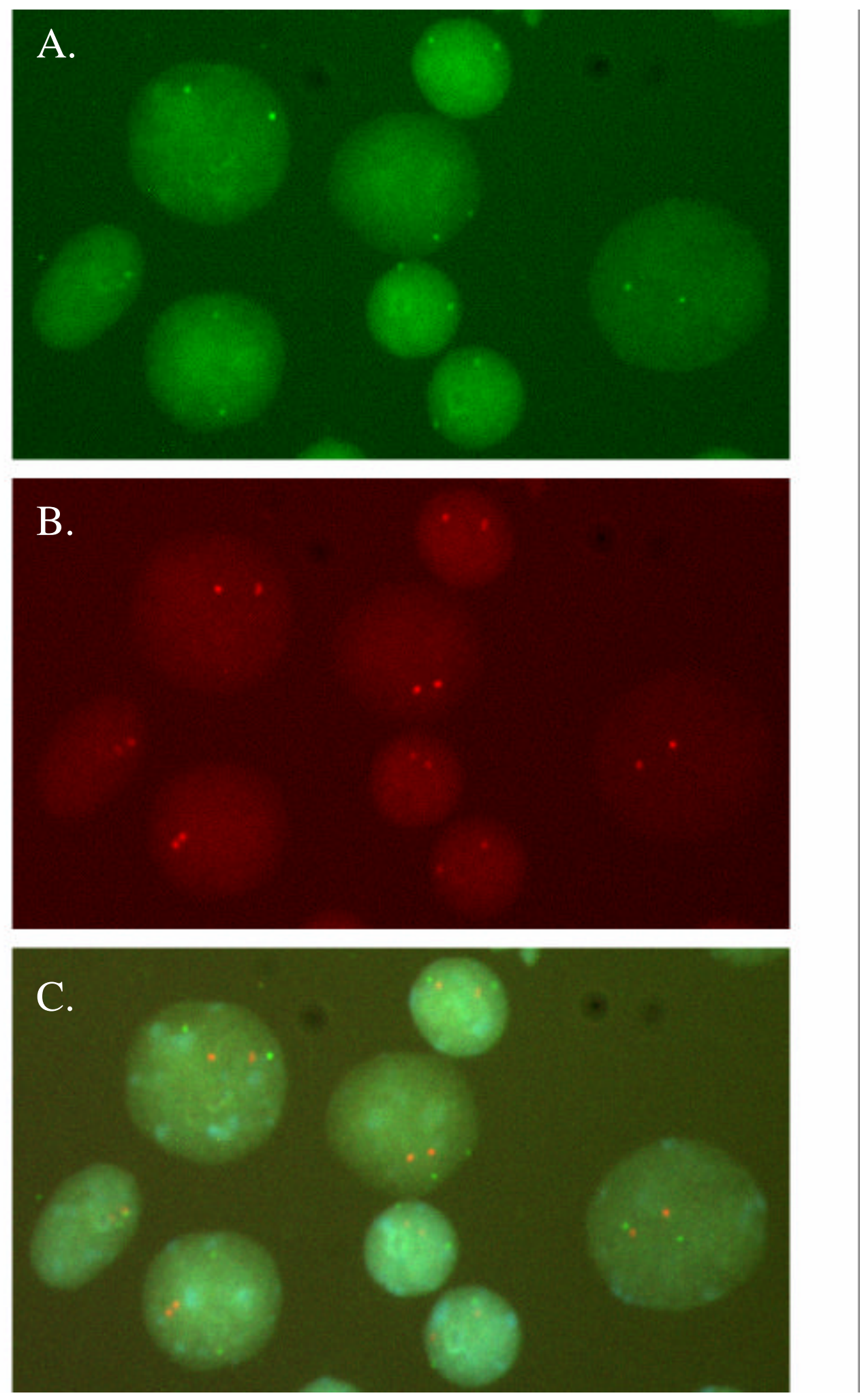

Figure 38. Interphase FISH Control Using Telomere Probes for 3p and 3q. (A) The 3p probes are visualized with FITC. (B) The 3q probes are visualized with TRITC. (C) The overlay of FITC and TRITC probes on a DAPI counterstain confirms 2 signals per cell for the control nuclei. 

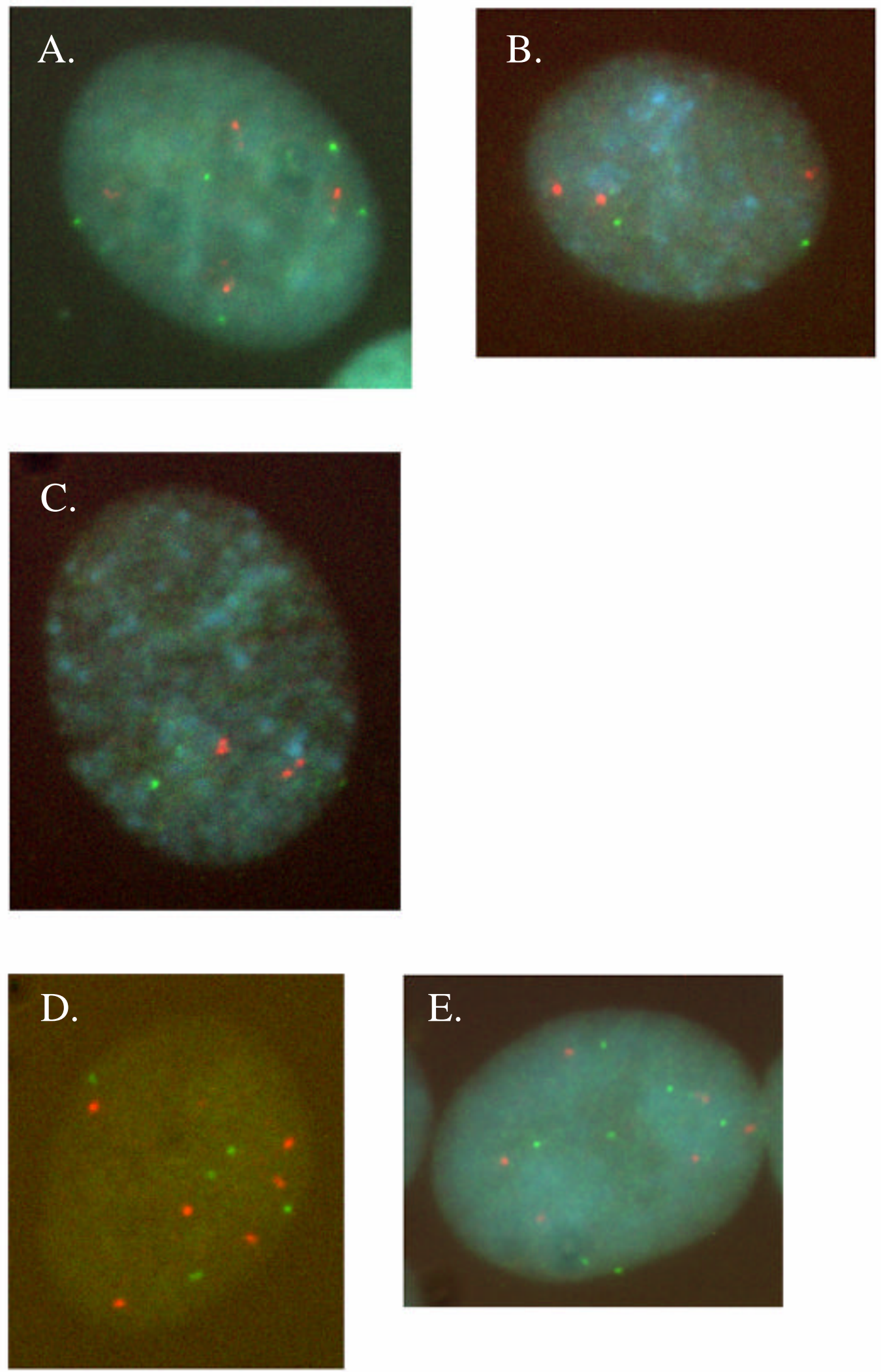

Figure 39. Telomere Probe Signals $(3 p$ and $3 q)$ in Uveal Melanoma Cell Lines. Examples from the cell line (A) MEL270; (B) MEL285; (C) MEL290; (D) OCM-1; (E) OCM-3. 


\title{
CURRICULUM VITAE
}

\author{
Jason Scott White \\ 9926 Woodburn Road \\ Silver Spring, MD 20901
}

\section{EDUCATION}

West Virginia University, Morg antown, WV

Ph.D. Genetics 2003

Franklin and Marshall College, Lancaster, PA

B.A. Biology 1998

\section{PROFESSIONAL EXPERIENCE}

2001-2002 Student Intern, Division of Quantitative Pathology, Armed Forces Institute of Pathology, Washington, D.C.

2000 Research Biologist, Student Intern, American Registry of Pathology, Armed Forces Institute of Pathology, Washington, D.C.

2000 Teaching Assistant, Department of Biology, West Virginia University, Morgantown, WV

1999 Research Biologist, American Registry of Pathology, Armed Forces Institute of Pathology, Washington, D.C.

1998-1999 Teaching Assistant, Department of Biology, West Virginia University, Morgantown, WV

\author{
AWARDS AND HONORS \\ 2001-2002 Nathaniel F. Rodman, Jr. Fellowship in Genetics and Developmental Biology, West \\ Virginia University, Morgantown, West Virginia. \\ 2001 Genetic Toxicology Association Student Travel Award, 32 ${ }^{\text {nd }}$ Environmental Mutagen \\ Society Meeting, San Diego, California. \\ 2001 West Virginia University Doctoral Student Travel Award for the $32^{\text {nd }}$ Environmental \\ Mutagen Society Meeting, San Diego, California. \\ 2000 Outstanding Student Poster Award, $31^{\text {st }}$ Environmental Mutagen Society Meeting, New \\ Orleans, Louisiana. \\ 2000 West Virginia University Doctoral Student Travel Award for the $31^{\text {st }}$ Environmental \\ Mutagen Society Meeting, New Orleans, Louisiana.
}

\section{PROFESSIONAL ACTIVITIES}

1999-2002 Member of the Environmental Mutagen Society.

\section{RELEVANT GRADUATE COURSE WORK}

Advanced Biochemical Genetics

Basic Concepts of Modern Genetics

Biochemistry

Cytogenetics

Human Genetics

Population Genetics

\section{DEMONSTRATED SKILLS}

Cell culture of human cell lines.

Peripheral blood culture (human).

Metaphase chromosome preparation from peripheral blood (human).

Nuclei isolation and cytospin preparation fromfresh and archival tissue.

Fluorescence in situ Hybridization (FISH), including interphase FISH applications on fresh and archival tissue.

Tyramide Signal Amplification (TSA) Indirect ISH

Comparative Genome Hybridization (CGH) using antibody layering for indirect probe detection on fresh and archival tissue.

Degenerate oligonucleotide primed (DOP) PCR, bulk production of genomic painting probes for use in CGH.

Nick translation incorporating substituted nucleotides for DNA labeling: production of genomic probes for $\mathrm{CGH}$. 
Karyotyping of human metaphase spreads using inverse DAPI banding.

Spectral Karyotyping (SKY) (human).

Agarose gel electrophoresis with ethidium bromide staining to determine the size of DNA products.

Cloning of PCR products into plasmid vectors.

Transformation of bacteria using plasmids and selection of positive transformants.

Restriction enzyme digestion.

SDS-PAGE.

Southern blot.

\section{PUBLICATIONS (in press or submitted)}

Director-Myska AE, White JS and McLean IW (2001) Molecular Cytogenetic Characterization of Ten Uveal Melanoma Cell Lines. Proceedings of the Workshop on Prognostic Factors in Uveal Melanoma, Lieden, The Netherlands (in press).

White JS, Becker RL, McLean IW, Nath J, Director-Myska AE (2002) Molecular Cytogenetic Evaluation of Ten Uveal Melanoma Cell Lines. Cancer Res (submitted).

\section{ABSTRACTS}

White JS, McLean IW, Nath J, Becker RL, Director-Myska AE (2001) Molecular prognostic indicators of uveal melanoma: correlation of DNA sequence copy number aberrations with patient follow-up. Environmental and Molecular Mutagenesis 37 (Suppl 32):79.

Director-Myska AE, White JS, Niederkorn JY, McLean IW (2000) Characterization of Structural and Numerical Chromosome Aberrations in Uveal Melanoma Cell Lines using Spectral Karyotyping. Environmental and Molecular Mutagenesis 35 (Suppl 31):20.

White JS, Mclean IW, Nath J, Becker RL, Director-Myska AE (2000) A Characterization of the Chromosomal Aberrations of Uveal Melanoma Using Comparative Genomic Hybridization. Environmental and Molecular Mutagenesis 35 (Suppl 31):66.

\section{INVITED SEMINARS}

The Molecular Cytogenetics of Uveal Melanoma. Genetic Toxicology Association Meeting, University of Delaware, Newark, DE, October 2001. 